\title{
Multi-aperture photometry of extended IR sources with ISOPHOT
}

\section{The nature of extended IR emission of planetary Nebulae ${ }^{\star} \star \star \star$}

\author{
U. Klaas ${ }^{1}$, H. J. Walker ${ }^{2}$, T. G. Müller ${ }^{3}$, P. J. Richards ${ }^{2}$, and J. Schreiber ${ }^{1}$ \\ 1 Max-Planck-Institut für Astronomie, ISOPHOT Data Centre, Königstuhl 17, 69117 Heidelberg, Germany \\ e-mail: klaas@mpia.de \\ 2 CCLRC Rutherford Appleton Laboratory, Chilton, Didcot, Oxon OX11 0QX, UK \\ 3 Max-Planck-Institut für extraterrestrische Physik, Giessenbachstr., 85748 Garching, Germany
}

Received 14 April 2005 / Accepted 21 February 2006

\section{ABSTRACT}

\begin{abstract}
Context. ISOPHOT multi-aperture photometry is an efficient method to resolve compact sources or to detect extended emission down to relatively faint levels with single detectors in the wavelength range 3 to $100 \mu \mathrm{m}$.

Aims. Using ISOPHOT multi-aperture photometry and complementary ISO spectra and IR spectral energy distributions we discuss the nature of the extended IR emission of the two PNe NGC 6543 and NGC 7008.

Methods. In the on-line appendix we describe the data reduction, calibration and interpretation methods based on a simultaneous determination of the IR source and background contributions from the on-source multi-aperture sequences. Normalized profiles enable direct comparison with point source and flat-sky references. Modelling the intensity distribution offers a quantitative method to assess source extent and angular scales of the main structures and is helpful in reconstructing the total source flux, if the source extends beyond a radius of 1 arcmin. The photometric calibration is described and typical accuracies are derived. General uncertainty, quality and reliability issues are addressed, too. Transient fitting to non-stabilised signal time series, by means of combinations of exponential functions with different time constants, improves the actual average signals and reduces their uncertainty.

Results. The emission of NGC 6543 in the $3.6 \mu \mathrm{m}$ band coincides with the core region of the optical nebula and is homogeneously distributed. It is comprised of $65 \%$ continuum and $35 \%$ atomic hydrogen line emission. In the $12 \mu \mathrm{m}$ band a resolved but compact double source is surrounded by a fainter ring structure with all emission confined to the optical core region. Strong line emission of [ArIII] at $8.99 \mu \mathrm{m}$ and in particular [SIV] at $10.51 \mu \mathrm{m}$ shapes this spatial profile. The unresolved $60 \mu \mathrm{m}$ emission originates from dust. It is described by a modified (emissivity index $\beta=1.5$ ) blackbody with a temperature of $85 \mathrm{~K}$, suggesting that warm dust with a mass of $6.4 \times 10^{-4} M_{\odot}$ is mixed with the ionised gas. The gas-to-dust mass ratio is about 220 . The $25 \mu \mathrm{m}$ emission of NGC 7008 is characterised by a $F W H M$ of about $50^{\prime \prime}$ with an additional spot-like or ring-like enhancement at the bright rim of the optical nebula. The $60 \mu \mathrm{m}$ emission exhibits a similar shape, but is about twice as extended. Analysis of the spectral energy distribution suggests that the $25 \mu \mathrm{m}$ emission is associated with $120 \mathrm{~K}$ warm dust, while the $60 \mu \mathrm{m}$ emission is dominated by a second dust component with $55 \mathrm{~K}$. The dust mass associated with this latter component amounts to $1.2 \times 10^{-3} M_{\odot}$, significantly higher than previously derived. The gas-to-dust mass ratio is 59 which, compared to the average value of 160 for the Milky Way, hints at dust enrichment by this object.
\end{abstract}

Key words. techniques: photometric - astronomical data bases: miscellaneous - infrared: ISM -

planetary nebulae: individual: NGC 6543 - planetary nebulae: individual: NGC 7008 - methods: data analysis

\section{Introduction}

Planetary nebulae $(\mathrm{PNe})$ represent a short $\left(\sim 10^{4} \mathrm{yr}\right)$ phase of stellar evolution between the asymptotic giant branch (AGB) and white dwarfs for stars with initial masses between 1.5 and $8 M_{\odot}$. The AGB period is characterized by strong mass loss via stellar winds. The discovery of gas phase molecules as well as submicrometre-sized solid-state particles of various chemical compositions in the cool circumstellar envelopes of AGB stars supports the view that extensive molecular synthesis occurs during this brief phase of stellar evolution. The condensation of dust grains can lead to an almost complete obscuration of the star.

* Based on observations with ISO, an ESA project with instruments funded by ESA Member States (especially the PI countries: France, Germany, the Netherlands and the United Kingdom) and with the participation of ISAS and NASA.

$\star \star$ Appendix A is only available in electronic form at http://www.edpsciences.org
The high rate of mass loss on the AGB will eventually deplete the envelope of the star and gradually expose the hot core. The stellar temperature increases and when it reaches $25000 \mathrm{~K}$, the UV photons begin to photo-ionise the envelope creating the PN. Therefore, AGB stars and $\mathrm{PNe}$ are considered to play a major role in the chemical enrichment and photo-chemistry of the ISM (Kwok 2001, 2004).

The dust distribution probably shapes the wind and determines the morphology of the final PN shape (e.g. Reimers et al. 2000). In the case of PNe with highly focused lobes it is suggested that the wind is collimated by a disk (Kwok 2001). Since the envelopes of AGB stars are still highly symmetric, this shaping must set in during the so-called Proto Planetary Nebulae (PPN) phase (Kwok 2001).

In order to assess the amount of dust and its temperature, as well as its spatial distribution and association with the ionized gas, infrared observations are essential. IRAS provided 
broad band FIR photometry for several hundred known PNe (Pottasch et al. 1984). On the basis of IRAS point source photometry for a statistical sample of 233 PNe Lenzuni et al. (1989) concluded that dust grains undergo substantial evolution during the lifetime of a PN. In particular the grain size and total dust mass should decrease systematically with the nebular radius, whereas the number of emitting grains should increase. Stasińska \& Szcerba (1999) concluded that this was an artefact of the analysis by Lenzuni et al. (1989) and that the time scale for destruction of dust grains in PNe is larger than their life time.

In order to investigate the subjects addressed above, better spatially and spectrally resolved MIR and FIR data of PNe are necessary. This became possible with imaging MIR cameras on the Infared Space Observatory (ISO) (e.g. Persi et al. 1999) and recently on the Spitzer satellite (e.g. Hora et al. 2004; Su et al. 2004). In the FIR spatial resolution is still restricted due to the small sizes of the IR space telescopes. First attempts to resolve PNe in the FIR with the help of IRAS pointed observations were reported by Leene \& Pottasch (1988).

In the case of extended emission with a scale length of $\approx 1^{\prime}$ to $2^{\prime}$ around a compact central source, the ISOPHOT instrument (Lemke et al. 1996) onboard ISO (Kessler et al. 1996) had a multi-aperture photometry observing mode (Astronomical Observation Template, AOT PHT04) which used all or a subset of the 11 circular apertures with diameters between $5^{\prime \prime}$ and $180^{\prime \prime}$. This is an efficient way to trace the radial structure of PNe. In the on-line Appendix A we give a detailed description of the techniques for constructing the source profiles and deriving angular scales as well as determining fluxes from the multi-aperture sequences. In the commissioning of this observing mode two PNe, NGC 6543 and NGC 7008, were observed at various MIR and FIR wavelengths. Here we present the results of these observations applying our novel methods outlined in Appendix A. We determine the extent of the MIR and FIR emission. With complementary ISOPHOT photometry and spectroscopy we clarify the nature of the emission, and derive dust temperatures and dust masses for the two objects. As a baseline we used the standard Offline Processing (OLP) Auto Analysis products from the ISO Data Archive (Kessler et al. 2003) for our analysis. The data processing steps leading to these products are fully described in Laureijs et al. (2003). However, we applied a revised uncertainty calculation which is outlined in Sect. A.1.3 of the online Appendix. The impact by non-stable signals (transients) will be addressed when discussing the individual profiles - in this case we performed additional evaluation with the ISOPHOT Interactive Analysis (PIA ${ }^{1}$, Gabriel et al. 1997).

\section{Extended IR emission of NGC 6543}

NGC 6543, also called the Cat's Eye Nebula, has been extensively studied in the optical with the Hubble Space Telescope (see e.g. Balick 2004). It has a strikingly complex, symmetric, and bright core of angular diameter of $\approx 25^{\prime \prime}$ and consists of a crossed pair of ellipses. Outside this core a system of at least nine regularly spaced concentric circular rings can be seen (Balick et al. 2001). It is surrounded by a large, patchy, filamentary halo of $\Theta \approx 300^{\prime \prime}$ (Balick et al. 1992). All of these features are mainly due to gas emission in the hydrogen recombination lines (Balmer series) and low to intermediate ionisation lines of oxygen and nitrogen ([OI], [NII], [OIII]).

${ }^{1}$ PIA is a joint development by the ESA Astrophysics Division and the ISOPHOT consortium led by the Max-Planck-Institut für Astronomie, Heidelberg.
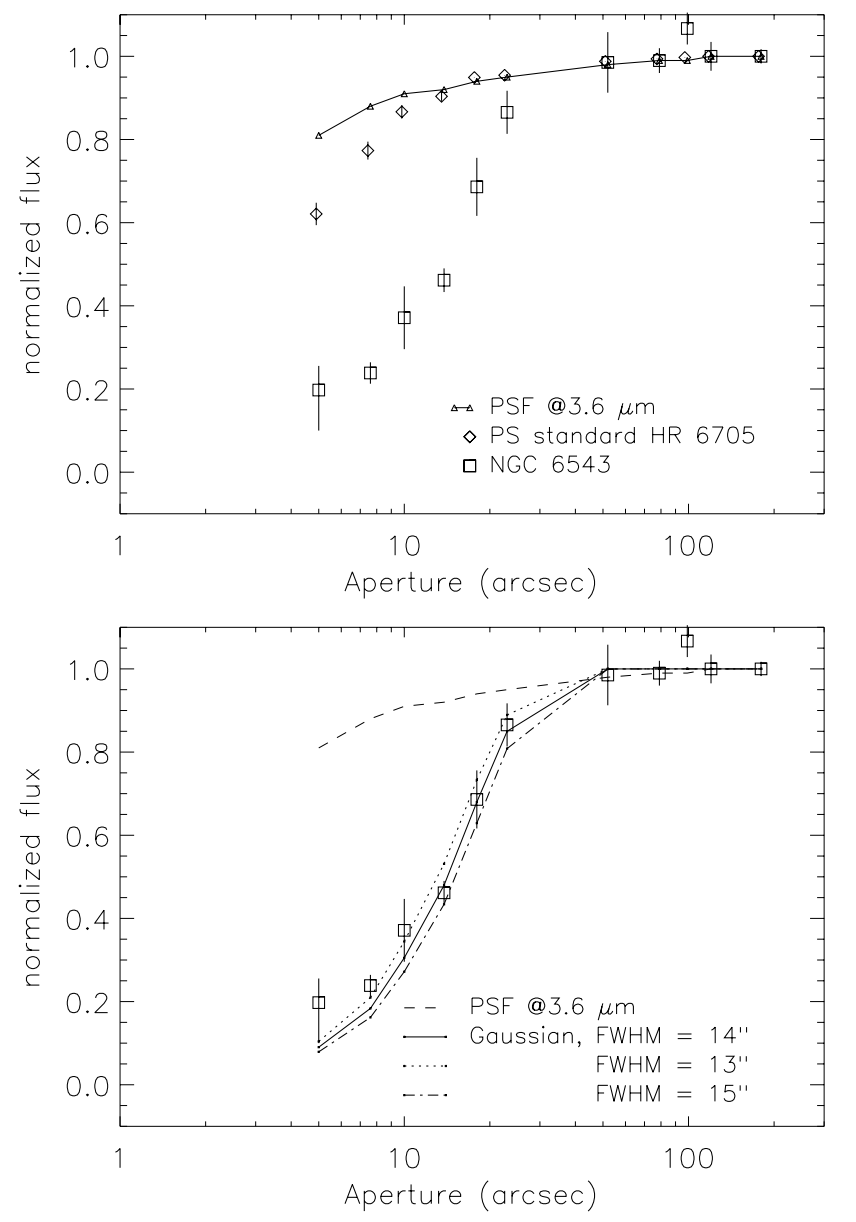

Fig. 1. Top: measured multi-aperture profile of NGC 6543 at $3.6 \mu \mathrm{m}$. The profiles of the theoretical PSF and the point source HR 6705 are shown for comparison. Bottom: the measured profile is fitted by a Gaussian intensity distribution with 3 different FWHM.

Three ISOPHOT multi-aperture sequences were performed on NGC 6543, at $3.6 \mu \mathrm{m}$ (ISO TDT No. 05702401), $12 \mu \mathrm{m}$ (ISO TDT No. 05702402), and $60 \mu \mathrm{m}$ (ISO TDT No. 05702403). For details of the observations see Table A.6 in the on-line appendix. The pointings of all three sequences were within 1". 1 of the central position listed in SIMBAD, RA $=17^{\mathrm{h}} 58^{\mathrm{m}} 33.423^{\mathrm{s}}$ and Dec $=+66^{\mathrm{d}} 37^{\prime} 59^{\prime \prime} .52(2000)$.

\section{1. $3.6 \mu m$ profile}

The signal time series of the $3.6 \mu \mathrm{m}$ sequence are displayed in Fig. A.11 in the online appendix. The data quality report in the ISO Data Archive associated with this TDT indicates unstable signals due to signal transients for the $5^{\prime \prime}$ and $79^{\prime \prime}$ apertures. In the case of the $5^{\prime \prime}$ aperture it appears that there is a drift downwards which is not fully accounted for by the average signal. Since there are only eight signals for this aperture we refrain from performing a transient correction, but increase the lower uncertainty range by $20 \%$. In the case of the $79^{\prime \prime}$ aperture signals no significant trend is obvious. We therefore use the ISO Data Archive product generated by OLP V10.0 for our analysis.

Figure 1 shows the ISOPHOT $3.6 \mu \mathrm{m}$ multi-aperture profile. The source-to-background contrast in the $180^{\prime \prime}$ aperture, $\frac{S_{180}^{\text {source }}}{S_{180}^{\mathrm{bg}}}$, is $700 \%$, so the background subtraction has no impact on the 


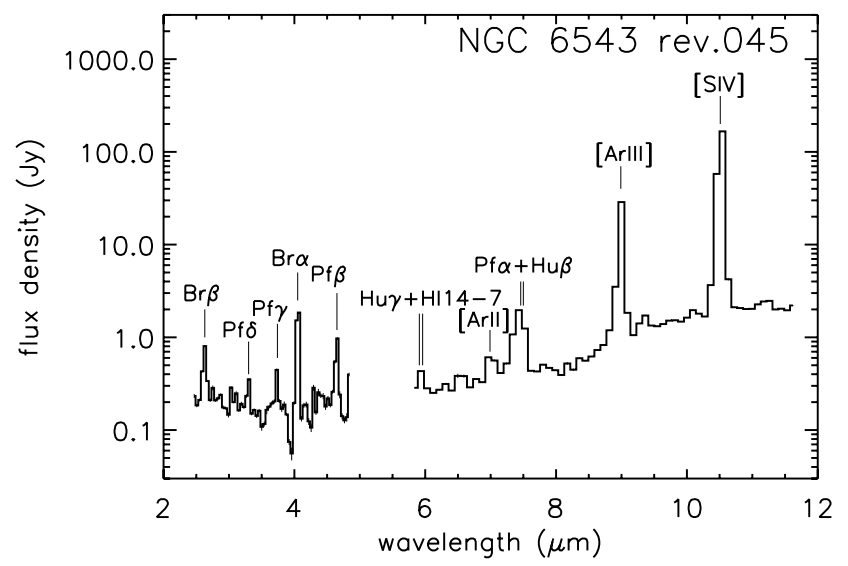

Fig. 2. ISOPHOT-S spectrum of NGC 6543. Clearly identified hydrogen recombination lines and forbidden lines are labelled.

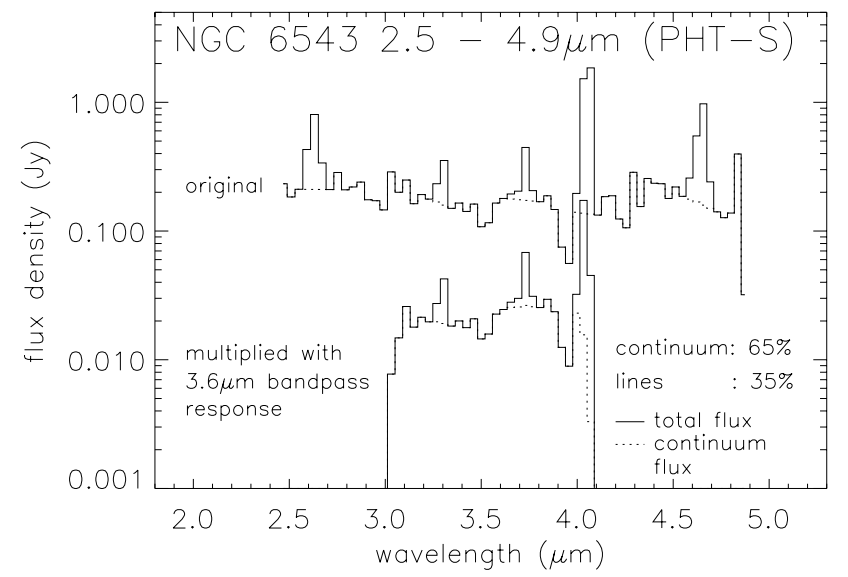

Fig. 3. Enlarged view of the spectrum of NGC 6543 between $2.5 \mu \mathrm{m}$ and $4.9 \mu \mathrm{m}$. The continuum level underneath the hydrogen recombination lines is indicated by dashed lines. The spectrum has been multiplied with the relative spectral response of the $3.6 \mu \mathrm{m}$ bandpass. The relative contributions to the total $3.6 \mu \mathrm{m}$ flux are $65 \%$ by the continuum and $35 \%$ by the hydrogen recombination lines $\operatorname{Pf} \delta, \operatorname{Pf} \gamma$, and $\operatorname{Br} \alpha$.

resulting source profile. The integrated flux (no color correction) in this aperture is $0.37 \pm 0.05 \mathrm{Jy}$.

We find a very good fit of this profile by a Gaussian intensity distribution with $F W H M=14^{\prime \prime} \pm 1^{\prime \prime}$. The emission in the $3.6 \mu \mathrm{m}$ band is therefore concentrated in the core region. The positive deviation of the $99^{\prime \prime}$ aperture point may indicate contribution by emission in the ring between $60^{\prime \prime}$ and $90^{\prime \prime}$ radius. Indeed, recent Spitzer IRAC images (Hora et al. 2004) show that there are a lot of cometary-shaped "flocculi" around the main nebula, also clearly visible in the IRAC $3.6 \mu \mathrm{m}$ band. However, we make no attempt to model this complex structure in our multi-aperture sequences.

Figure 2 shows an ISOPHOT-S spectrum of NGC 6543. With its $24^{\prime \prime} \times 24^{\prime \prime}$ aperture size it is quite well matched to the core region. The spectrum shows that the line emission in the $3.6 \mu \mathrm{m}$ band is dominated by hydrogen recombination lines. We find no indication for a significant $\mathrm{H}_{2}$ emission, as suggested by Hora et al. (2004), as a contributor to the emission in the IRAC 5.8 and $8.0 \mu \mathrm{m}$ bands. Figure 3 shows the relative contributions of the continuum and the emission lines to the total flux in the $3.6 \mu \mathrm{m}$ band.

We conclude that the emission in the $3.6 \mu \mathrm{m}$ band is homogeneously distributed emission from continuum $(65 \%)$ and hydrogen recombination lines (35\%), mainly $\operatorname{Br} \alpha, \operatorname{Pf} \gamma$ and $\operatorname{Pf} \delta$, from the central 14" of the core region. Since the continuum is flat or rising towards longer wavelengths a contribution by very small grain emission to the hot central star radiation is suggested.

\section{2. $12 \mu \mathrm{m}$ profile}

The signal time series of the $12 \mu \mathrm{m}$ sequence are displayed in Fig. A.1 in the online appendix. The data quality report in the ISO Data Archive associated with this TDT indicates unstable signals due to signal transients for the $79^{\prime \prime}, 120^{\prime \prime}$ and $180^{\prime \prime}$ apertures. We therefore performed a detailed transient analysis with PIA, which is described in Sect. A.1.4 of the online appendix.

Figure 4 shows the transient corrected ISOPHOT $12 \mu \mathrm{m}$ multi-aperture profile. The source-to-background contrast in the $180^{\prime \prime}$ aperture, $\frac{S_{180}^{\text {source }}}{S_{180}^{\text {bg }}}$, is only $28 \%$, so the background subtraction is crucial here. The integrated flux (no color correction) in this aperture is $7.0 \pm 1.0 \mathrm{Jy}$.

A Gaussian intensity distribution with $F W H M=13^{\prime \prime} \pm$ $2^{\prime \prime}$ provides a reasonable fit to the profile. However, for the inner part at $r=5^{\prime \prime}$ the profile appears to be sharper and the 23" aperture point indicates a somewhat wider profile. Note that the $F W H M$ at $12 \mu \mathrm{m}$ is the same as the one at $3.6 \mu \mathrm{m}$, hence the emission in this band is confined to the core region, too.

Indeed the measured profile can be better fit by models consisting of three components, as shown in Fig. 5 for four possible examples. The parameters of the four models are summarized in Table 1. As outlined in online Appendix A, there is no unique solution because of the integration in the circular aperture. However, common features for all four models are a central, practically unresolved bright core, a compact or (partly) extended component at an offset distance of 7" and faint, probably extended emission concentrated around a radius of $17^{\prime \prime}$.

In the case of this $12 \mu \mathrm{m}$ profile of NGC 6543 we can compare our models with a real image taken in the wavelength range which can be constructed from an ISOCAM CVF scan (Blommaert et al. 2003). This observation has been described in Persi et al. (1999). From the ISO Data Archive (Kessler et al. 2003) we have retrieved homogeneously processed data sets, imported there as Highly Processed Data Products (HPDP, Boulanger et al. 2005) and used the final CVF data cube for our investigation. This data cube comprises the wavelength ranges $5.0-9.0 \mu \mathrm{m}$ and $9.0-16.5 \mu \mathrm{m}$, hence covers fully the ISOPHOT $12 \mu \mathrm{m}$ bandpass (cf. Fig. 7).

The applied pixel scale was $11^{\prime \prime} 5$, hence the $32 \times 32$ image covers a spatial area of $48^{\prime \prime} \times 48^{\prime \prime}$, with the largest radius from the centre along the diagonal being 33"' 9 . Figure 6 shows the spatial structure in the three bright lines inside the ISOPHOT-P $12 \mu \mathrm{m}$ bandpass, with the [SIV]-line being by far the most dominant one, and the continuum at $11.8 \mu \mathrm{m}$. The [SIV]-line shows a double peak structure with 7" separation and one peak close to the SIMBAD position. A similar morphology appears in the [ArIII]-line, but the peaks are closer together. The lower excitation NeII-line has one pronounced maximum which is located north of the SIMBAD position.

In the top panel of Fig. 7, the spectrum integrated over the ISOPHOT-S aperture size as indicated in Fig. 6 is shown. We have convolved each CVF spectral image with the ISOPHOT-P $12 \mu \mathrm{m}$ bandpass response and obtained a synthetic ISOCAM $12 \mu \mathrm{m}$ (broad band) image, which is depicted in the bottom panel of Fig. 7. As expected, it closely resembles the morphology of the image in the peak of the [SIV]-line. 

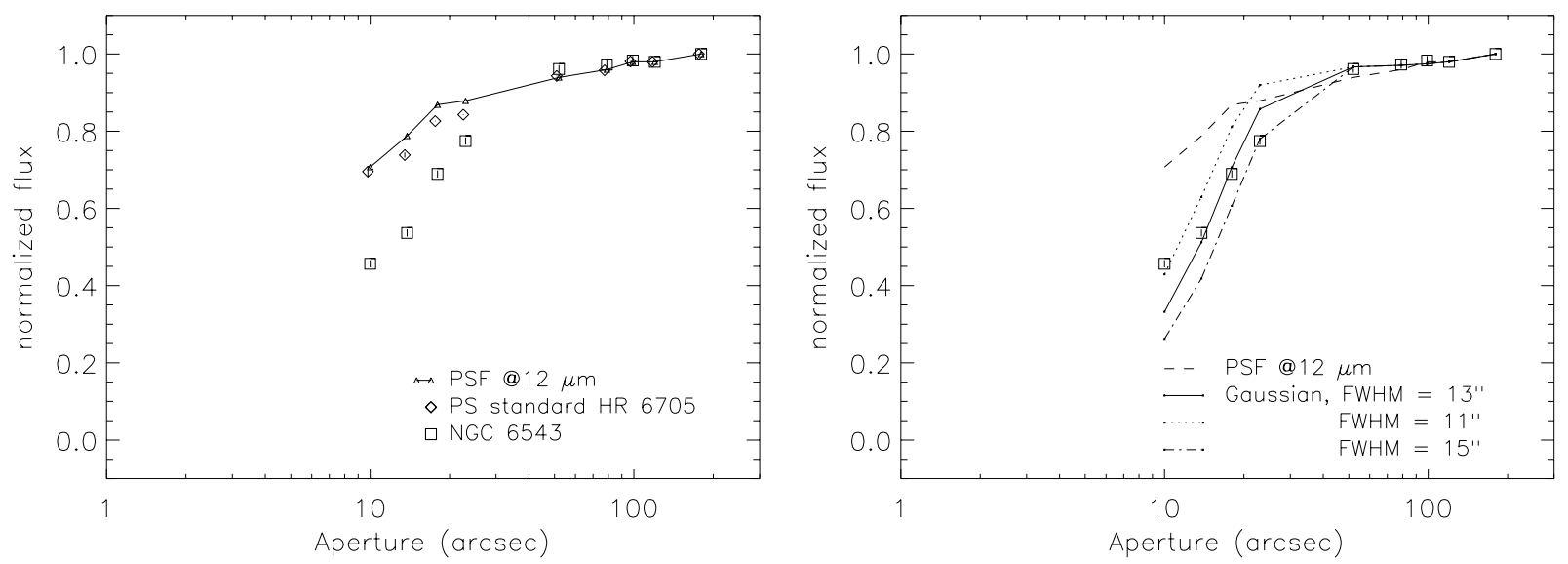

Fig. 4. Left: multi-aperture profile of NGC 6543 at $12 \mu \mathrm{m}$. The profiles of the theoretical PSF and the point source HR 6705 are shown for comparison. Right: fit of the measured profile by a simple Gaussian distribution. Distributions for 3 different $F W H M$ are drawn.
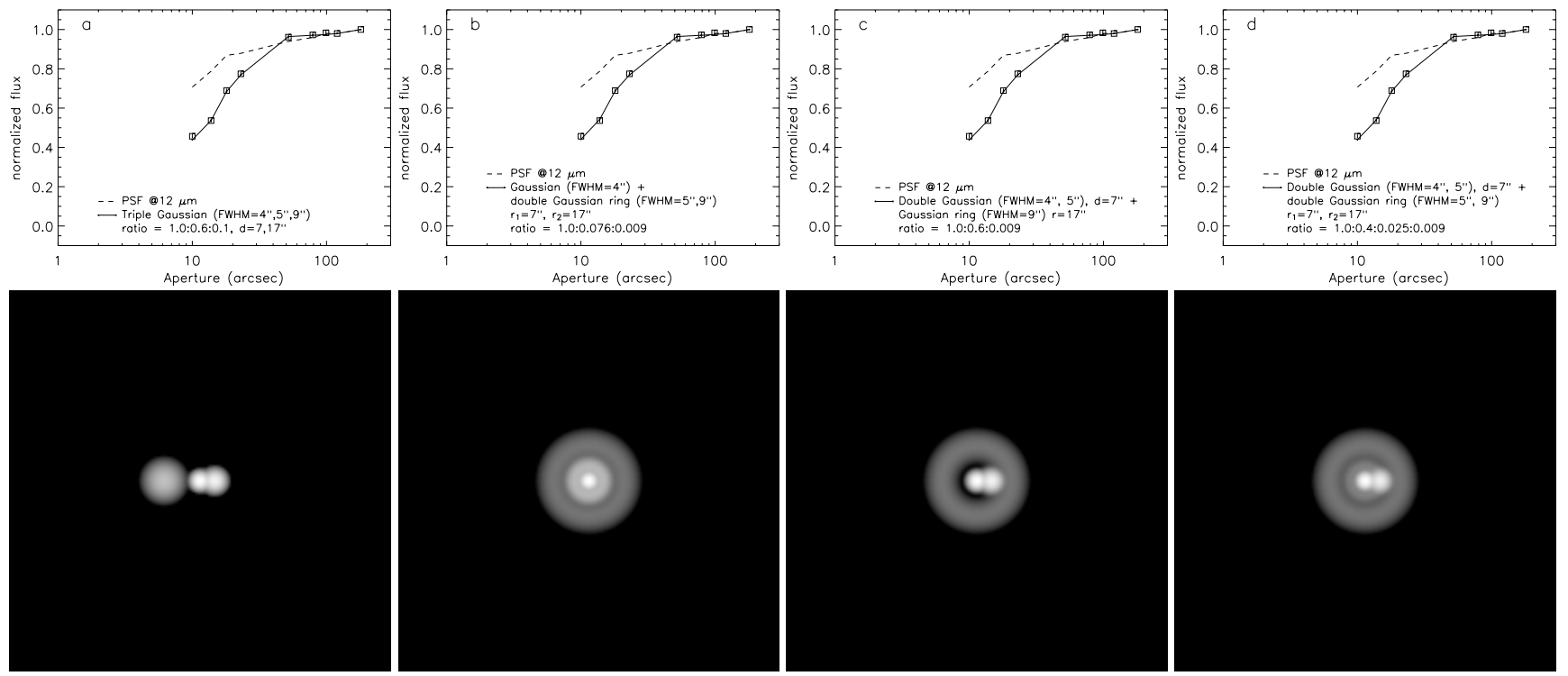

Fig. 5. Modelling of the NGC $654312 \mu \mathrm{m}$ multi-aperture profile. Upper panels: fits of different models to the measured data points; lower panels: grey-scale representation of the modelled intensity distributions - the size of the grey-scale images is $180^{\prime \prime} \times 180^{\prime \prime}$. a) Model 1 : triple Gaussian, the central one with $F W H M=4^{\prime \prime}$, the second $7^{\prime \prime}$ offset component with $F W H M=5^{\prime \prime}$ and the third $17^{\prime \prime}$ offset component with $F W H M=9^{\prime \prime}$. b) Model 2: central Gaussian with two Gaussian rings with radii of 7" and 17", respectively; FWHM are as for case a. c) Model 3: double Gaussian with second component offset by 7" with regard to the centre position plus a Gaussian ring with radius $17^{\prime \prime} ; F W H M$ are as for case a. d) Model 4: double Gaussian with second component offset by 7" with regard to the centre position plus a double Gaussian ring with radii of 7" and 17", respectively; the inner ring is at the same distance and has the same FWHM as the compact offset source, all FWHM are as for case a. Details of the model parameters have been compiled in Table 1 .

Table 1. Compilation of model parameters for the NGC $654312 \mu \mathrm{m}$ profile (see Figs. 4 and 5).

\begin{tabular}{|c|c|c|c|c|c|c|}
\hline $\begin{array}{c}\text { Model } \\
\quad \#\end{array}$ & Component 1 & $\begin{array}{l}\text { Parameter } \\
\text { and range }\end{array}$ & Component 2 & $\begin{array}{l}\text { Parameter } \\
\text { and range }\end{array}$ & Component 3 & $\begin{array}{l}\text { Parameter } \\
\text { and range }\end{array}$ \\
\hline 1 & central Gaussian & $F W H M=4^{\prime \prime} 0 \pm 0 . ' 1$ & offset Gaussian & $\begin{array}{l}F W H M=5^{\prime \prime} .0 \pm 0.5 \\
d=70^{\prime \prime} 0 \pm 0.2 \\
l^{\prime} .2\end{array}$ & offset Gaussian & $\begin{array}{l}F W H M=9 . .0 \pm 0.5 \\
d=17^{\prime \prime} 0 \pm 2 . .0 \\
I^{\prime \prime}=0.1 \pm 0.01\end{array}$ \\
\hline 2 & central Gaussian & $F W H M=4{ }^{\prime \prime} 0 \pm 0{ }^{\prime} 1$ & Gaussian ring & $\begin{array}{l}I_{\text {rel }}=0.0 \pm 0.1 \\
F W H M=5^{\prime \prime} 0 \pm 00^{\prime} 5 \\
r=77^{\prime \prime} 0 \pm 0,2\end{array}$ & Gaussian ring & $\begin{array}{l}\text { rel }=0.1 \pm 0.01 \\
F W H M=9 .{ }^{\prime} 0 \pm 0.5 \\
r=17^{\prime \prime} 0 \pm 22^{\prime \prime} 0\end{array}$ \\
\hline 3 & central Gaussian & $\begin{array}{l}I_{\mathrm{rel}}=1.0 \\
F W H M=4{ }^{\prime \prime} 0 \pm 0 .^{\prime} 1\end{array}$ & offset Gaussian & $\begin{array}{l}I_{\mathrm{rel}}=0.076 \pm 0.013 \\
F W H M=5^{\prime \prime} 0 \pm 00^{\prime} 5 \\
d=7^{\prime \prime} 0 \pm 0.0^{\prime} 2\end{array}$ & Gaussian ring & $\begin{array}{l}I_{\text {rel }}=0.009 \pm 0.001 \\
F W H M=9 !^{\prime} 0 \pm 0.5 \\
r=17^{\prime \prime} 0 \pm 22^{\prime \prime} 0\end{array}$ \\
\hline 4 & central Gaussian & $\begin{array}{l}I_{\mathrm{rel}}=1.0 \\
F W H M=4{ }^{\prime \prime} 0 \pm 0 .{ }^{\prime} 1\end{array}$ & $\begin{array}{l}\text { offset Gaussian/ } \\
\text { Gaussian ring }\end{array}$ & $\begin{array}{l}I_{\text {rel }}=0.6 \pm 0.1 \\
F W H M=5^{\prime \prime} 0 \pm 0 .{ }^{\prime} 5\end{array}$ & Gaussian ring & $\begin{array}{l}I_{\mathrm{rel}}=0.009 \pm 0.001 \\
F W H M=9 .^{\prime} 0 \pm 0 .{ }^{\prime} 5\end{array}$ \\
\hline & & $I_{\text {rel }}=1.0$ & & $\begin{array}{l}d / r=7{ }^{\prime \prime} 0 \pm 0 .{ }^{\prime} 2 \\
I_{\mathrm{rel}}=0.4 / 0.025\end{array}$ & & $\begin{array}{l}r=17^{\prime \prime} .0 \pm 22^{\prime \prime} 0 \\
I_{\text {rel }}=0.009 \pm 0.001\end{array}$ \\
\hline
\end{tabular}



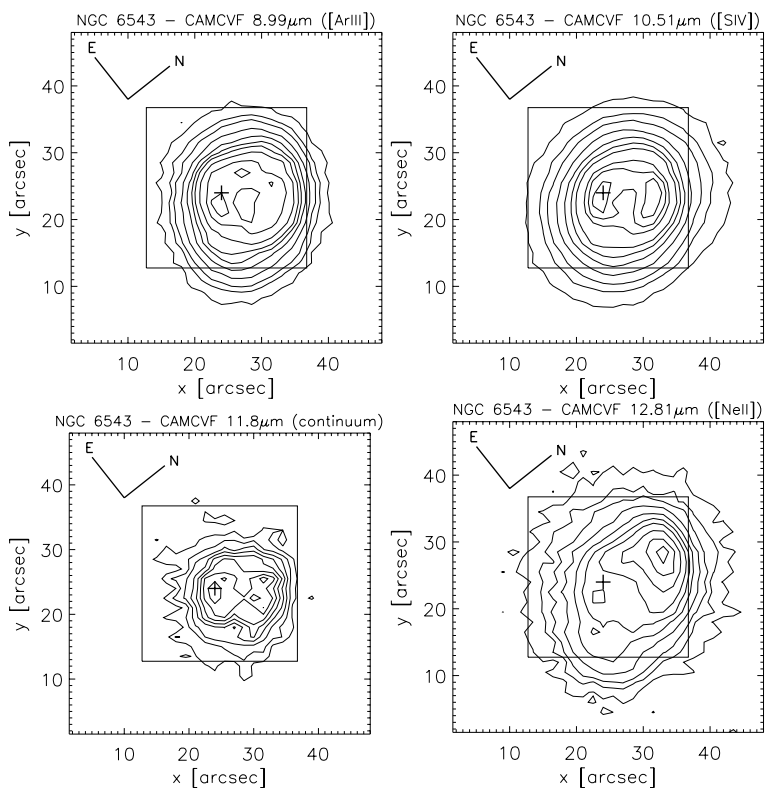

Fig. 6. ISOCAM CVF images of NGC 6543 in the three brightest emission lines ([ArIII] $8.99 \mu \mathrm{m}$, [SIV] $10.51 \mu \mathrm{m}$, [NeII] $12.8 \mu \mathrm{m}$ ) covered by the ISOPHOT-P $12 \mu \mathrm{m}$ bandpass and in the continuum at $11.8 \mu \mathrm{m}$. The SIMBAD position is indicated by the cross. The area of the ISOPHOT-S $24^{\prime \prime} \times 24^{\prime \prime}$ aperture is indicated by the square.

On this reference image we simulated an ISOPHOT multiaperture sequence around the SIMBAD position and the resulting profile is displayed by the solid line in the top graph of Fig. 8. While qualitatively there is good agreement with the measured ISOPHOT profile, quantitatively the profiles do not match within the error bars of the ISOPHOT measurement. In the inner part the ISOCAM profile implies a broader source $\left(F W H M \approx 17^{\prime \prime}\right)$ than the ISOPHOT profile (Gaussian $F W H M=10^{\prime \prime}-13^{\prime \prime}$ ).

The best corresponding case of our ISOPHOT multi-aperture profile modelling would be the central Gaussian with the offset Gaussian and the double Gaussian ring. The morphological feature of the two central peaks at a distance of $7^{\prime \prime}$ is well reproduced by our model. However, the side by side comparison of contour plots with the same intensity levels shows the much shallower intensity gradients and broader profiles in the ISOCAM image. The two ring features of the ISOPHOT model are below the displayed intensity levels (see Table 1 for the maximum intensities of these features).

We have investigated which uncertainties might cause the discrepancy between the ISOPHOT and ISOCAM profiles. A comparison of the ISOPHOT-S spectrum (Fig. 2) and the spatially integrated ISOCAM-CVF spectrum (Fig. 7) shows comparable continuum levels. Since, due to the two times lower spectral resolution of the CVF-spectrum, the peak intensity in the [SIV]-line is lower than in the ISOPHOT-S spectrum, we have determined the integrated line flux after continuum subtraction. With $F_{[\mathrm{SIV}]}=5.5 \times 10^{-13} \mathrm{~W} \mathrm{~m}^{-2}$ the ISOPHOT-S line flux is 1.67 times higher than the integrated ISOCAM-CVF flux. One reason may be that the transient correction in the ISOCAM data processing (Boulanger et al. 2005) could not completely recover the very strong rise of the flux in the peaks during the CVF scan. As a test we have increased the flux for all pixels in the [SIV]-line image which are above $70 \%$ of the maximum flux until the total ISOCAM-CVF flux in the [SIV]-line became equal to the ISOPHOT-S line flux. The resulting profile is shown by the dotted line in Fig. 8. It is somewhat lifted up and
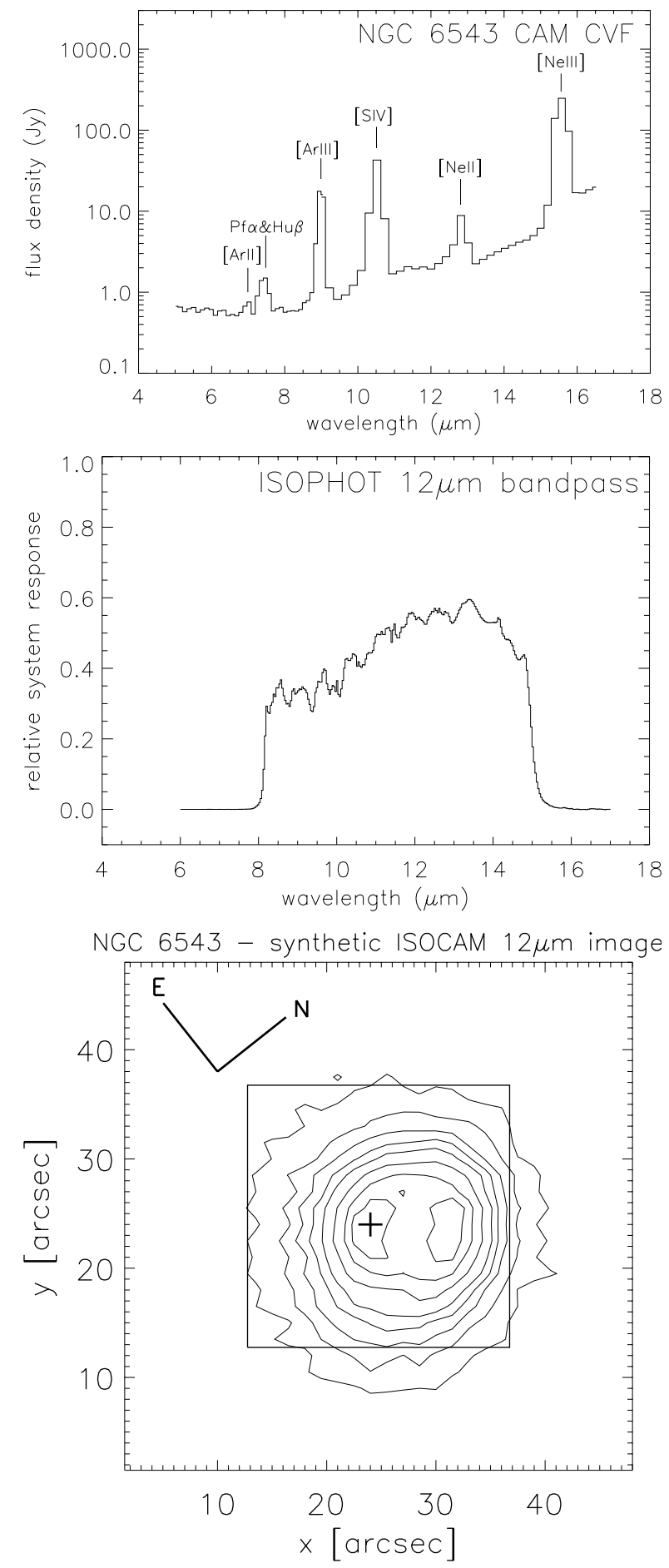

Fig. 7. Top: ISOCAM CVF spectrum of NGC 6543. The flux was integrated over an area of $24^{\prime \prime} \times 24^{\prime \prime}$ (corresponding to the aperture size of ISOPHOT-S) around the SIMBAD position. Middle: relative spectral system response of the ISOPHOT-P $12 \mu \mathrm{m}$ bandpass. Bottom: synthetic ISOCAM image after convolution of the spectral scan with the ISOPHOT-P $12 \mu \mathrm{m}$ bandpass response. The SIMBAD position is indicated by the cross. The area of the ISOPHOT-S $24^{\prime \prime} \times 24^{\prime \prime}$ aperture is indicated by the square.

hence closer to the measured ISOPHOT profile, however, the corrected ISOCAM image still implies a significantly broader source structure for the smaller apertures.

The reason for the final discrepancy remains open. There is the possibility that the [SIV]-line flux in the ISOPHOT-S 

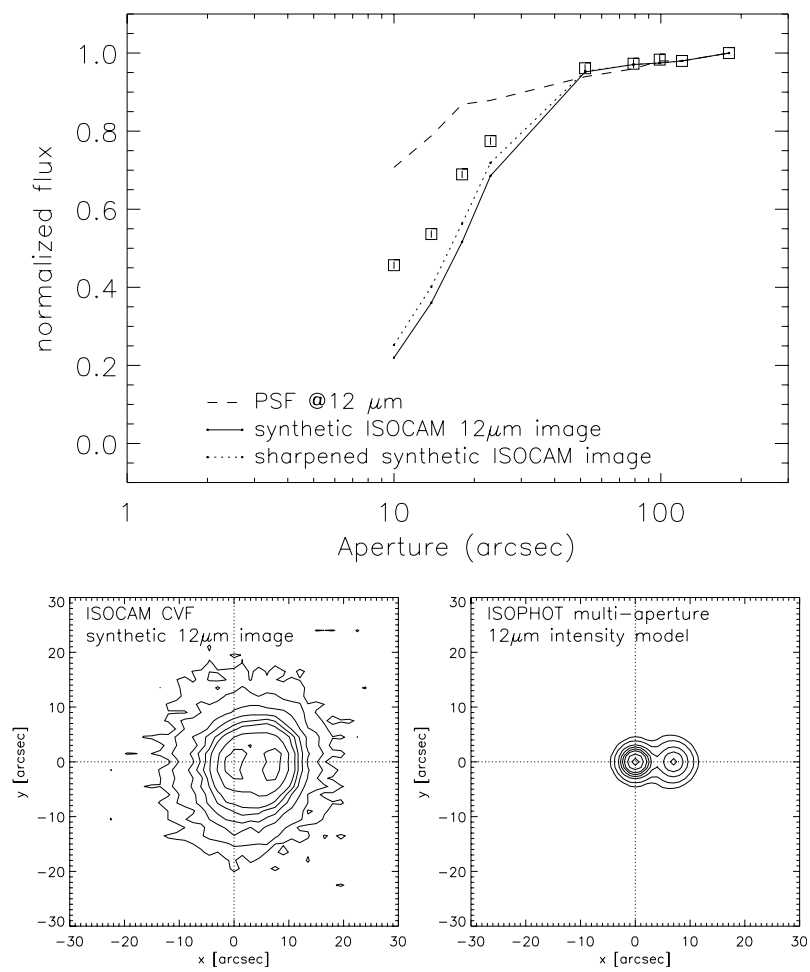

Fig. 8. Top panel: resulting aperture profile using the synthetic ISOCAM $12 \mu \mathrm{m}$ (CVF scan convolved with ISOPHOT $12 \mu \mathrm{m}$ bandpass response) image (solid line); the measured ISOPHOT multi-aperture profile is indicated by the squares. The dashed line represents an ISOCAM CVF image profile with sharpened peaks in the [SIV]-line image (see text for a more detailed description). Bottom panel: contour plot representation of the synthetic ISOCAM $12 \mu \mathrm{m}$ image (left). For comparison we show the best corresponding model of the ISOPHOT multi-aperture profile (Fig. 5d) which is the central Gaussian with the offset Gaussian and the double Gaussian ring (right). The two ring features are, however, below the displayed contour level range which is $0.05,0.1,0.2,0.3,0.4,0.5,0.7$ and 0.9 of the maximum intensity for both images. The crossed dotted lines indicate the SIMBAD position as the centre of the multi-aperture measurement.

spectrum is underestimated due to reduced off-centre aperture efficiency for the extended source emission which would require an even higher correction for the CVF-scan. On the other hand, the discrepancy may be due to differences in the footprints of both instruments unknown to us (e.g. due to straylight or ghost features) or a systematic uncertainty of the multi-aperture mode caused by the strong non-linear aperture response for the $12 \mu \mathrm{m}$ band (cf. online Appendix A.1.3).

\section{3. $60 \mu m$ profile}

The signal time series of the $60 \mu \mathrm{m}$ sequence are displayed in Fig. A.11 in the online Appendix A. The data quality report in the ISO Data Archive associated with this TDT does not indicate unstable signals for any aperture. We therefore use the ISO Data Archive product generated by OLP V10.0 for our analysis.

Figure 9 shows the ISOPHOT $60 \mu \mathrm{m}$ multi-aperture profile. The background contribution is totally neglegible, so that the background subtraction has no impact on the resulting source profile. The integrated flux (no color correction) in the $180^{\prime \prime}$ aperture is $143 \pm 5.5 \mathrm{Jy}$.

It can be seen from the profile that the source behaves pointlike down to the $52^{\prime \prime}$ aperture, i.e. the $60 \mu \mathrm{m}$ emission is at

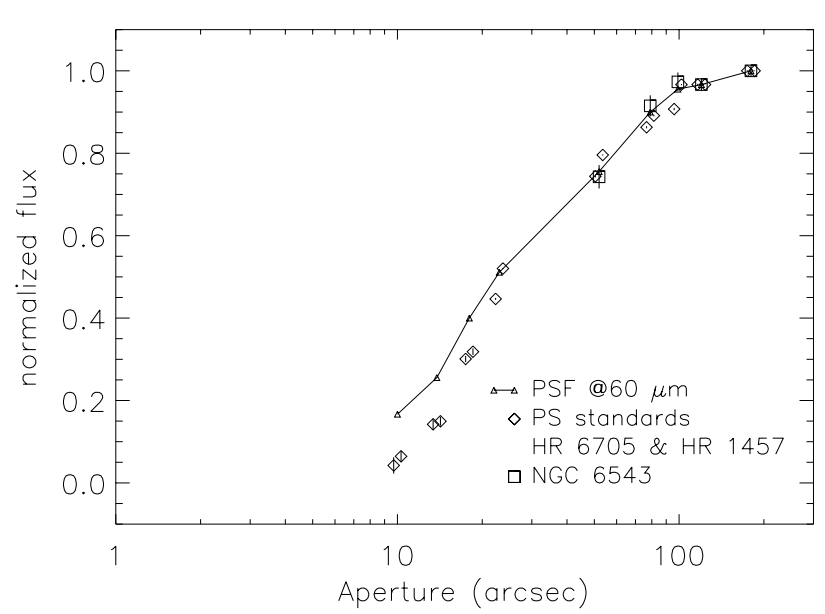

Fig. 9. Multi-aperture profile of NGC 6543 at $60 \mu \mathrm{m}$. The profiles of the theoretical PSF and the point sources HR 6705 and HR 1457 are shown for comparison.

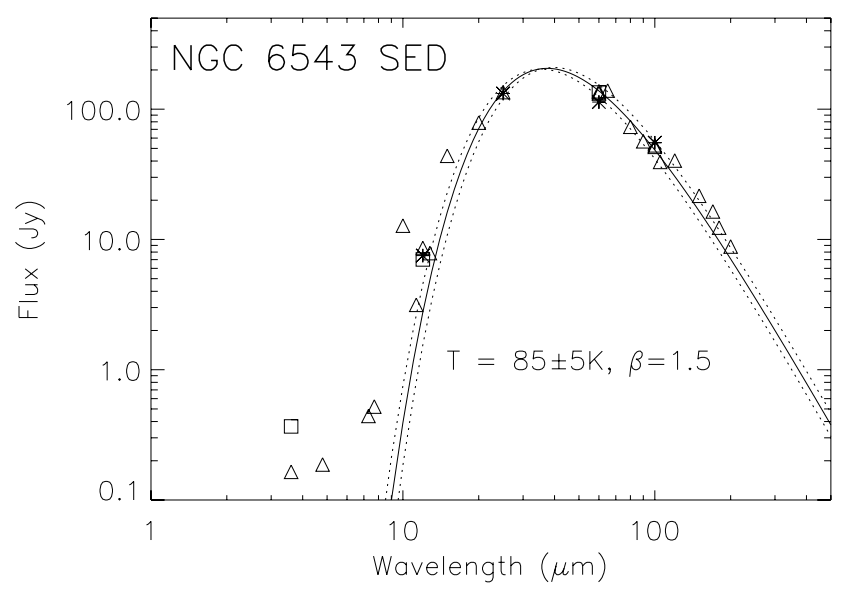

Fig. 10. Spectral energy distribution of NGC 6543 from ISOPHOT multi-filter measurements (triangles), the beam correction has been performed assuming a point source. The results from the multi-aperture photometry (180" aperture) are also drawn (squares). Asterisks indicate IRAS photometry. Fluxes are compiled in Table 2. Photometric accuracies are about $10 \%$. The solid line represents the emission of a modified blackbody with the indicated parameters which was eyeball fitted to the data points for $\lambda \geq 20 \mu \mathrm{m}$. The dotted lines outline the temperature uncertainty of $\pm 5 \mathrm{~K}$.

least concentrated within a radius of $<26^{\prime \prime}$. From IRAS Chopped Photometric Channel (CPC) observations Leene \& Pottasch (1988) found that NGC 6543 is extended at 50 and $100 \mu \mathrm{m}$. They derived a deconvolved size of about $53^{\prime \prime}$ at $50 \mu \mathrm{m}$. However, the deconvolution at different contour levels indicated that the source is sharper bound than a Gaussian and the deconvolved size was only an upper limit to the true size. We cannot exclude a slight extension of NGC 6543 at $60 \mu \mathrm{m}$, but our results put a stronger constraint on the upper limit and restrict the emission to the core region.

\subsection{Photometry}

Figure 10 shows the IR SED of NGC 6543 obtained with ISOPHOT multi-filter photometry (see Table 2 for the compilation of the fluxes, including the multi-aperture and IRAS measurements). This shows that the FIR emission is dominated by the continuum emission from dust. From LWS spectral scans in 
Table 2. Photometry of NGC 6543 from IRAS (FSC), ISOPHOT multiaperture (ma) photometry and ISOPHOT multi-filter (mf) photometry. Multi-aperture fluxes are derived from the signal in the 180" aperture. The multi-filter photometry applied different aperture sizes which are given in a separate column. The fluxes are corrected for beam size effects by dividing with the PSF fraction $f_{\text {aper }}^{\text {psf }}$ of the aperture. For $\lambda_{\mathrm{c}} \geq 25 \mu \mathrm{m}$ all fluxes are color corrected with regard to a modified blackbody spectrum $F_{v} \propto v^{1.5} \cdot B(v, T=85 \mathrm{~K})$.

\begin{tabular}{|c|c|c|c|c|}
\hline $\begin{array}{l}\lambda_{\mathrm{c}} \\
(\mu \mathrm{m})\end{array}$ & $\begin{array}{l}\text { IRAS } \\
\text { flux } \\
(\mathrm{Jy})\end{array}$ & $\begin{array}{l}\text { ISOPHOT ma } \\
\text { flux } \\
(\mathrm{Jy})\end{array}$ & $\begin{array}{l}\text { ISOPHOT mf } \\
\text { flux } \\
\text { (Jy) }\end{array}$ & $\begin{array}{c}\text { mf aperture } \\
\text { diameter / size } \\
\left({ }^{\prime \prime}\right)\end{array}$ \\
\hline 3.6 & - & 0.37 & 0.16 & 23 \\
\hline 4.8 & - & - & 0.19 & 23 \\
\hline 7.3 & - & - & 0.44 & 23 \\
\hline 7.7 & - & - & 0.52 & 23 \\
\hline 10 & - & - & 12.8 & 52 \\
\hline 11.3 & - & - & 3.15 & 52 \\
\hline 12 & 7.52 & 7.02 & 8.60 & 52 \\
\hline 12.8 & - & - & 7.85 & 52 \\
\hline 15 & - & - & 43.8 & 52 \\
\hline 20 & - & - & 78.8 & 79 \\
\hline 25 & 132 & - & 135 & 79 \\
\hline 60 & 113 & 143 & 137 & 180 \\
\hline 60 & 113 & 143 & 137 & $138 \times 138$ \\
\hline 65 & - & - & 139 & $138 \times 138$ \\
\hline 80 & - & - & 73.2 & $138 \times 138$ \\
\hline 90 & - & - & 56.7 & $138 \times 138$ \\
\hline 100 & 55.3 & - & 50.3 & 180 \\
\hline 100 & 55.3 & - & 50.7 & $138 \times 138$ \\
\hline 105 & - & - & 53.9 & $138 \times 138$ \\
\hline 120 & - & - & 40.3 & $184 \times 184$ \\
\hline 150 & - & - & 21.6 & $184 \times 184$ \\
\hline 170 & - & - & 16.4 & $184 \times 184$ \\
\hline 180 & - & - & 12.3 & $184 \times 184$ \\
\hline 200 & - & - & 8.82 & $184 \times 184$ \\
\hline
\end{tabular}

the ISO Data Archive (AOTs L01, Gry et al. 2003) we estimate that the brightest fine structure line, [OIII] at $51.8 \mu \mathrm{m}$ contributes $\approx 10 \%$ to the emission in the broad-band filter. The 10 and $15 \mu \mathrm{m}$ points clearly pop up above the continuum as outlined by the blackbody curve, which is explained by the strong line contributions of the $10.51 \mu \mathrm{m}$ [SIV] and $15.56 \mu \mathrm{m}$ [NeIII] lines.

\subsection{Dust properties}

We were able to fit the $20-200 \mu \mathrm{m}$ part of the SED with a single modified blackbody with a temperature of $85 \pm 5 \mathrm{~K}$ and an emissivity index of $\beta=1.5$. Lenzuni et al. (1989) derived a dust temperature of $92 \pm 0.9 \mathrm{~K}$ from IRAS 25,60 and $100 \mu$ m fluxes. Pottasch et al. (1984) obtained a dust temperature of $125 \mathrm{~K}$ by assuming blackbody emission with constant emissivity. The dust temperature is at the upper end of the range found in dust temperature determinations of evolved stars by Bowey et al. (2002) both from FIR continuum and the $69 \mu \mathrm{m}$ forsterite band peak location analysis. Bowey et al. (2002) also found that an emissivity index of $\beta=1.5$ gave the closest match between the continuum temperature estimates and the forsterite $69 \mu \mathrm{m}$ peak temperatures.

The dust mass is calculated with the standard formula

$M_{\text {dust }}=\frac{1}{\kappa_{850 \mu \mathrm{m}}(\beta)} \cdot \frac{D^{2} \cdot S_{850 \mu \mathrm{m}}}{B_{850 \mu \mathrm{m}}(T=85 \mathrm{~K})}$

with $\kappa_{850 \mu \mathrm{m}}(\beta=1.5)=1.595 \mathrm{~cm}^{2} \mathrm{~g}^{-1}$ adopting the scaling of the dust opacities with emissivity index $\beta$ as described in
Klaas et al. (2001) and using $\kappa_{850 \mu \mathrm{m}}(\beta=2)=0.865 \mathrm{~cm}^{2} \mathrm{~g}^{-1}$. $S_{850 \mu \mathrm{m}}=0.063 \mathrm{Jy}$ is the $850 \mu \mathrm{m}$ flux of the modified blackbody fit and $B_{850 \mu \mathrm{m}}(T=85 \mathrm{~K})$ the $850 \mu \mathrm{m}$ flux of a blackbody of $85 \mathrm{~K}$. With a distance $D=1.0 \mathrm{kpc}$ (Cahn et al. 1992; Balick et al. $2001)$, this results in a dust mass $M_{\text {dust }}=6.4 \pm 2.0 \times 10^{-4} M_{\odot}$ from which the $60 \mu \mathrm{m}$ emission originates. Lenzuni et al. (1989) derived a dust mass of $6.9 \times 10^{-4} M_{\odot}$, although their dust temperature was slightly higher $(92 \mathrm{~K})$ and they used a distance of $640 \mathrm{pc}$, taken from Daub (1982). Pottasch et al. (1984) obtained a dust mass of $1.1 \times 10^{-4} M_{\odot}$ for a distance of $600 \mathrm{pc}$. The span of dust masses in the statistical samples of Lenzuni et al. (1989) and Pottasch et al. (1984) is between $3 \times 10^{-6} M_{\odot}$ and $3 \times 10^{-2} M_{\odot}$ for 233 objects and between $1 \times 10^{-5} M_{\odot}$ and $3.5 \times 10^{-3} M_{\odot}$ for 45 objects, respectively. This means that the dust mass of NGC 6543 is in the upper half of the observed range.

Estimates of the gas mass for NGC 6543 are in the range of around $0.12 M_{\odot}$ to $0.17 M_{\odot}$ (Bianchi et al. 1986). This implies a dust-to-gas ratio in the range $\frac{M_{\text {dust }}}{M_{\text {gas }}}=5.3-3.8 \times 10^{-3}$. Thus, NGC 6543 has a near-normal or elevated gas-to-dust ratio (190-260), compared to the Milky Way value.

\section{Extended IR emission of NGC 7008}

NGC 7008 is an elliptical type PN (Górny et al. 1997). In fact its morphology resembles a $\mathrm{C}$ and displays several bright knots (e.g. Hua \& Louise 1972). Leene \& Pottasch (1988) give a size of about $65^{\prime \prime} \times 52^{\prime \prime}$ for the bright part and $90^{\prime \prime} \times 78^{\prime \prime}$ for the weak halo. Jewitt et al. (1986) measured a size of $80^{\prime \prime}$ at $10 \%$ and a halo of $120^{\prime \prime}$ in diameter. Its infrared emission was resolved by IRAS, the Small Scale Structure Catalog source X2059+543 is associated with the IRAS PSC source 20590+5420.

Two ISOPHOT multi-aperture sequences were performed on NGC 7008, at $25 \mu \mathrm{m}$ (ISO TDT No. 05702610) and $60 \mu \mathrm{m}$ (ISO TDT No. 05702611). For details of the observations see Table A.6 in the on-line appendix. The pointings of the two sequences were within 3 '. $^{\prime \prime}$ and 6.' 8 , respectively, of the central position listed in SIMBAD, RA $=21^{\mathrm{h}} 00^{\mathrm{m}} 32.5^{\mathrm{s}}$ and Dec $=+54^{\mathrm{d}} 32^{\prime} 36^{\prime \prime}(2000)$.

\section{1. $25 \mu \mathrm{m}$ profile}

The signal time series of the $25 \mu \mathrm{m}$ sequence are displayed in Fig. A.11 in the online Appendix A. The data quality report in the ISO Data Archive associated with this TDT does not indicate unstable signals for any aperture. We therefore use the ISO Data Archive product generated by OLP V10.0 for our analysis.

Figure 11 shows the ISOPHOT $25 \mu \mathrm{m}$ multi-aperture profile. The source-to-background contrast in the $180^{\prime \prime}$ aperture, $\frac{S_{180}^{\text {source }}}{S_{180}^{\text {bg }}}$, is $130 \%$. The source is clearly extended at $25 \mu \mathrm{m}$.

A relatively good fit of the profile is achieved with a Gaussian profile with a $F W H M$ of $55^{\prime \prime} \pm 11^{\prime \prime} 5$, whereby we have taken into account the $4^{\prime \prime}$ pointing-offset with regard to the central position of the source (Fig. 12a). However, while the fit is perfect for the inner part (i.e. $23^{\prime \prime}$ and 52" apertures) the wing of the Gaussian is too broad to fit the profile and the $79^{\prime \prime}$ and $99^{\prime \prime}$ aperture points lie above the Gaussian profile. A better fit to the measured profile is achieved with a somewhat narrower central Gaussian ( $F W H M$ of 49.'5) plus an offset Gaussian at a distance of $33^{\prime \prime}$ with a FWHM of $16^{\prime \prime}$, which compensates the flatter shape of the central Gaussian for the two inner apertures (Fig. 12b). An equivalent profile is achieved by replacing the off-centre Gaussian spot by a Gaussian ring with a relative amplitude of 0.107 at a radius 


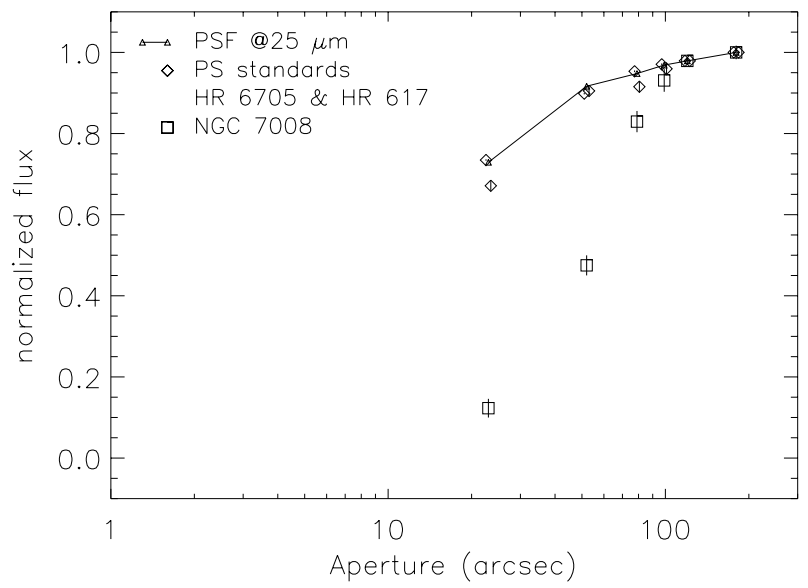

Fig. 11. Multi-aperture profile of NGC 7008 at $25 \mu \mathrm{m}$. The profiles of the theoretical PSF and the point sources HR 6705 and HR 617 are shown for comparison.

of $33^{\prime \prime}$. Since the step in aperture size is relatively large between the $52^{\prime \prime}$ and $79^{\prime \prime}$ apertures an alternative applicable model is a narrower offset Gaussian, but with higher amplitude to recover the total intensity in this annulus. Therefore either an offset Gaussian spot with a FWHM of $9^{\prime \prime}$ and a relative intensity of 3.8 or a Gaussian ring with the same $F W H M$ and a relative intensity of 0.166 at a slightly larger distance/radius of $35^{\prime \prime}$ gives an equally good fit (Fig. 12c). The parameters of the 5 models, including the ranges which are consistent with the error bars of the measurement, are compiled in Table 3.

From the measured profile we can exclude models where the emission is restricted to a ring-like structure and the centre is free of emission, similar to the optical appearance. Figure 13 shows as "case a" just the ring-like structure of model 3 in Table 3 . The resulting profile shows significant differences from the measured one. If the radius is made smaller and the width of the ring is enlarged, the measured profile can be adjusted, as demonstrated in "case b". However, the cut through the intensity distribution shows, that the emission fills up the central region and it resembles a broadened Gaussian.

The picture which arises from our multi-aperture analysis is that the dust emitting at $25 \mu \mathrm{m}$ is relatively homogeneously distributed over the area outlined by the bright optical nebula with either spot-like or ring-like enhancement at the rim where also the maximum of the optical emission comes from.

\section{2. $60 \mu m$ profile}

The signal time series of the $60 \mu \mathrm{m}$ sequence are displayed in Fig. A.11 in the online Appendix A. The data quality report in the ISO Data Archive associated with this TDT indicates unstable signals due to signal transients for the 52" aperture. Inspection of the transient curve suggests that the signal series is about to converge and that the final signal level is only a few percent higher than the one determined by the drift recognition. This does not lead to any strong modification of the source profile and we use the ISO Data Archive product generated by OLP V10.0 for our analysis.

Figure 14 shows the ISOPHOT $60 \mu \mathrm{m}$ multi-aperture profile. The source-to-background contrast in the $180^{\prime \prime}$ aperture, $\frac{S_{180}^{\text {source }}}{S_{180}^{\text {bg }}}$, is $200 \%$. The source is clearly extended also at $60 \mu \mathrm{m}$.

Again, a relatively good first fit of the profile is achieved with a Gaussian profile, this time with a $F W H M$ of $100^{\prime \prime} \pm 10^{\prime \prime}$, including the 7 " pointing-offset with respect to the central position of the source (Fig. 15a). A first result is therefore, that the $60 \mu \mathrm{m}$ emission is nearly twice as extended as the $25 \mu \mathrm{m}$ emission, arguing for a decrease of the dust temperature with radius.

The single Gaussian is somewhat too narrow for the inner part of the profile and too wide in the wing. Again, as for the $25 \mu \mathrm{m}$ profile, a double Gaussian provides a better fit. The central Gaussian has a FWHM of $90^{\prime \prime}$. The second component is offset by 33", and either spot-like or distributed over a ring, with a $F W H M$ of $25^{\prime \prime}$. The parameters of the models, including the ranges which are consistent with the error bars of the measurement are compiled in Table 4.

The offset Gaussian is at the same distance to the centre as the offset Gaussian in the $25 \mu \mathrm{m}$ models, which confirms the enhancement of the emission at the border of the optically bright nebula. Relative to the intensity of the central emission, the $60 \mu \mathrm{m}$ ring emission is brighter than the $25 \mu \mathrm{m}$ ring emission and it extends into the halo area.

Leene \& Pottasch (1988) found extended FIR emission of NGC 7008 by IRAS CPC observations. They derived a deconvolved size at $10 \%$ intensity of about $110^{\prime \prime}$ at $50 \mu \mathrm{m}$. They concluded that this is an upper limit, because a true PN has a much steeper brightness distribution at its edge than a gaussian profile, which they used for the deconvolution. The deconvolved size at $50 \%$ intensity, which would correspond to the $F W H M$ of our fits, is $\approx 70^{\prime \prime}$, somewhat smaller than what our models suggest. A source size of 1.7 (=102") is suggested from the IRAS Small Scale Structure Catalog.

\subsection{Photometry}

The Gaussian model profiles for NGC 7008 are so extended that they need a reconstruction of the total flux with the technique described in online Appendix A.3.2, using the intensity ratio $\frac{I_{120}^{\text {model }}}{I_{180}^{\text {model }}}$ of the model. Table 5 summarizes the fluxes and compares them with the various IRAS results. At $25 \mu \mathrm{m}$ the variation among the two models and the direct determination of the flux in the $180^{\prime \prime}$ aperture is small. The ISOPHOT multi-aperture fluxes are consistent with the IRAS Small Scale Structure Catalog flux within about $15 \%$. At $60 \mu \mathrm{m}$ the variation in resulting fluxes is larger. The best correspondence with the IRAS fluxes of both the Small Scale Structure Catalog and the Chopped Photometric Channel is achieved for the double Gaussian.

\subsection{Dust properties}

A dust temperature of $77 \pm 2.9 \mathrm{~K}$ was derived by Lenzuni et al. (1989) from their fit to the IRAS 25, 60 and $100 \mu$ m fluxes. Stasińska \& Szcerba (1999) obtained 83 K, including a color correction of the IRAS fluxes. Pottasch et al. (1984) derived $95 \mathrm{~K}$ with a pure blackbody fit. Tajitsu \& Tamura (1998) list a dust temperature of $146 \mathrm{~K}$ derived from IRAS point source fluxes to which they applied an aperture correction, because the nebula diameter is larger than $25^{\prime \prime}$. This resulted in a $25 \mu \mathrm{m}$ flux prediction of $207 \mathrm{Jy}$ (color corrected), a value which we cannot confirm by our extended source photometry.

Figure 16 shows the spectral energy distribution of NGC 7008 resulting from ISOPHOT multi-aperture and IRAS extended source photometry. Due to the few data points the SED is not as well constrained as in the case of NGC 6543. However, the detailed SED of NGC 6543 gave us some hints for the current solution: We have adopted the same emissivity index, $\beta=1.5$ which was also the prefered one by Bowey et al. (2002). The 

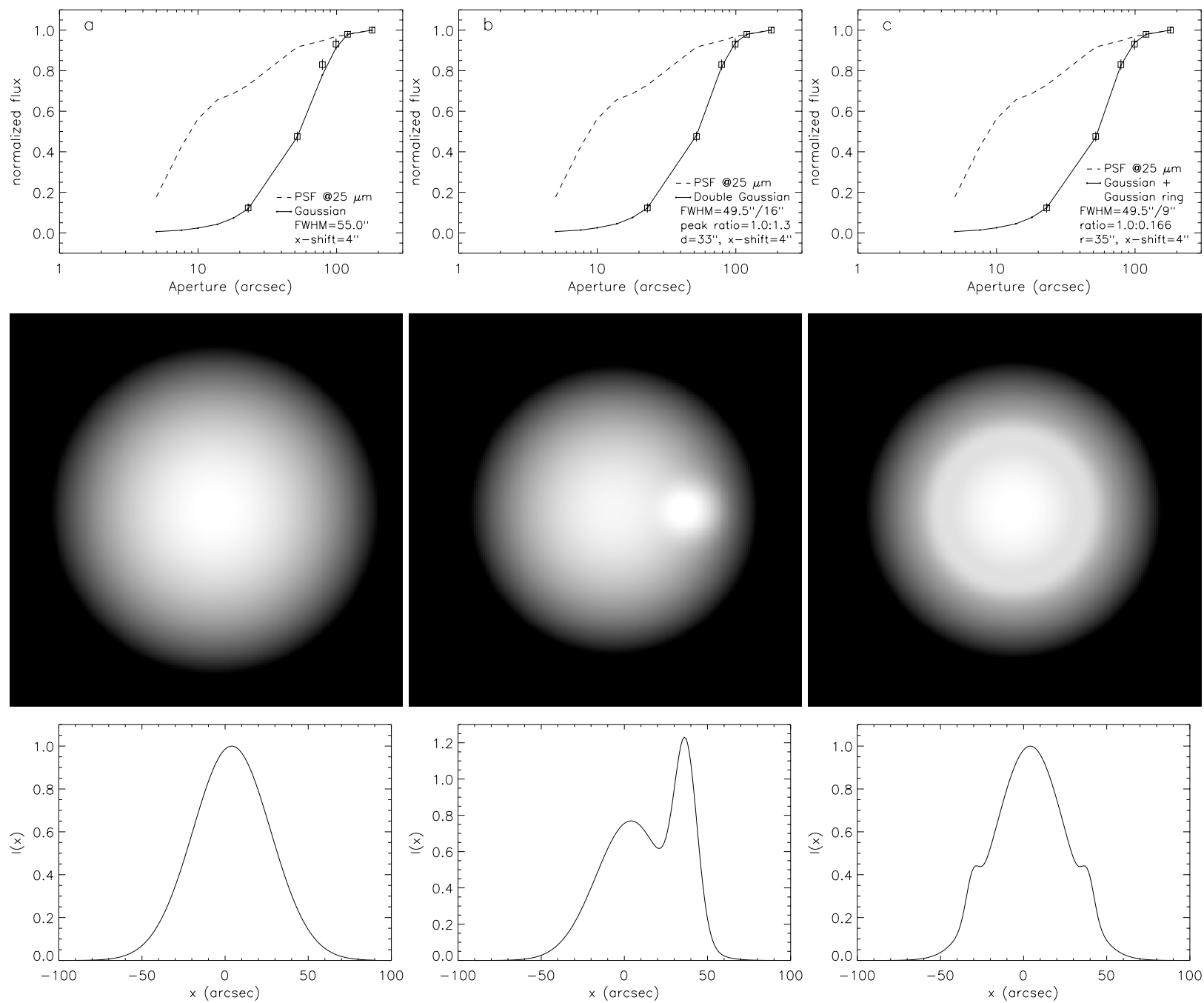

Fig. 12. Modelling of the NGC $700825 \mu$ m multi-aperture profile. Details of the model parameters have been compiled in Table 3. Upper panels: fits of different models to the measured data points; middle panels: grey-scale representation of the modelled intensity distributions - the size of the grey-scale images is $180^{\prime \prime} \times 180^{\prime \prime}$; lower panels: cut through the (normalized) intensity distributions along the $x$-axis. a) Model 1 : Gaussian with $F W H M=55^{\prime \prime}$ shifted by $4^{\prime \prime}$ off the centre. b) Model 2: Gaussian with $F W H M=49$ '. 5 plus offset $\left(d=33^{\prime \prime}\right)$ Gaussian with $F W H M=16^{\prime \prime}$ both shifted by $4^{\prime \prime}$ off the centre; the peak ratio of the two Gaussians is 1:1.3. c) Model 5: Central Gaussian with FWHM=49.5 plus offset Gaussian ring $\left(r=35^{\prime \prime}\right)$ with $F W H M=9^{\prime \prime}$ both shifted by $4^{\prime \prime}$ off the centre; the peak ratio of the central Gaussian and the ring is 1:0.166.

Table 3. Compilation of model parameters for the NGC $700825 \mu \mathrm{m}$ profile (see Figs. 11 and 12 for models 1,2 and 5). The positional offset indicates that the total intensity distribution has been shifted (arbitrarily in the $+x$-direction) with regard to the origin of the multi-aperture sequence taking into account the offset with regard to the SIMBAD position.

\begin{tabular}{|c|c|c|c|c|c|}
\hline $\begin{array}{l}\text { Model } \\
\quad \#\end{array}$ & Component 1 & $\begin{array}{l}\text { Parameter } \\
\text { and range }\end{array}$ & Component 2 & $\begin{array}{l}\text { Parameter } \\
\text { and range }\end{array}$ & $\begin{array}{l}\text { Positional } \\
\text { offset }\end{array}$ \\
\hline 1 & central Gaussian & $F W H M=555^{\prime \prime} 0 \pm 2{ }^{\prime \prime} 0$ & _- & - & $4^{\prime \prime}$ \\
\hline 2 & central Gaussian & $\begin{array}{l}F W H M=49.5 \pm 1^{\prime \prime} .5 \\
I_{\mathrm{rel}}=1.0\end{array}$ & offset Gaussian & $\begin{array}{l}F W H M=16^{\prime \prime} 0 \pm 33^{\prime \prime} 0 \\
d=33^{\prime \prime} 0 \pm 33^{\prime \prime} 0 \\
I_{\mathrm{rel}}=1.3 \pm 0.6\end{array}$ & $4^{\prime \prime}$ \\
\hline 3 & central Gaussian & $\begin{array}{l}F W H M=49.5 \pm 1 . .5 \\
I_{\mathrm{rel}}=1.0\end{array}$ & Gaussian ring & $\begin{array}{l}F W H M=166^{\prime \prime} 0 \pm 33^{\prime \prime} 0 \\
r=33^{\prime \prime} \cdot 0 \pm 3{ }^{\prime \prime} 0 \\
I_{\text {rel }}=0.107 \pm 0.050\end{array}$ & $4^{\prime \prime}$ \\
\hline 4 & central Gaussian & $F W H M=49 . .5 \pm 2 . .0$ & offset Gaussian & $\begin{array}{l}F W H M=95^{\prime} 0 \pm 1^{\prime \prime} 5 \\
d=35^{\prime \prime} 0 \pm 22^{\prime \prime} 5\end{array}$ & $4^{\prime \prime}$ \\
\hline 5 & central Gaussian & $\begin{array}{l}I_{\text {rel }}=1.0 \\
F W H M=49 . ' 5 \pm 2 . .0\end{array}$ & Gaussian ring & $\begin{array}{l}I_{\text {rel }}=3.8 \pm 1.4 \\
F W H M=9.0 \pm 1^{\prime \prime} .5 \\
r=35^{\prime \prime} 0 \pm 2, .5\end{array}$ & $4^{\prime \prime}$ \\
\hline & & $I_{\mathrm{rel}}=1.0$ & & $I_{\text {rel }}=0.167 \pm 0.062$ & \\
\hline
\end{tabular}



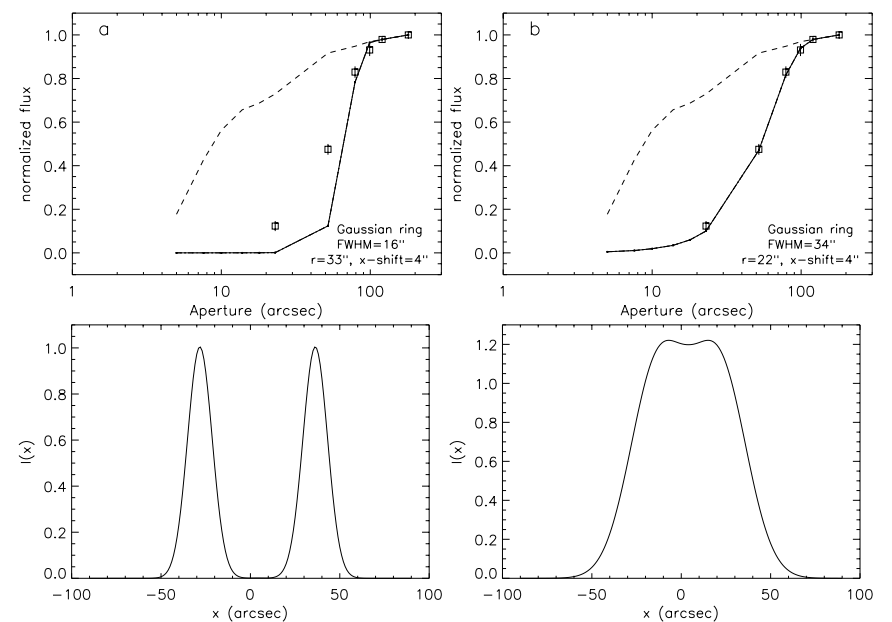

Fig. 13. Resulting profiles for NGC 7008, if the source is modelled only by a ring-like structure. Case a): ring structure of model 3 in Table 3, the centre is free of emission. Case b): ring structure that gives a good fit to the measured data points. The centre is filled with emission.

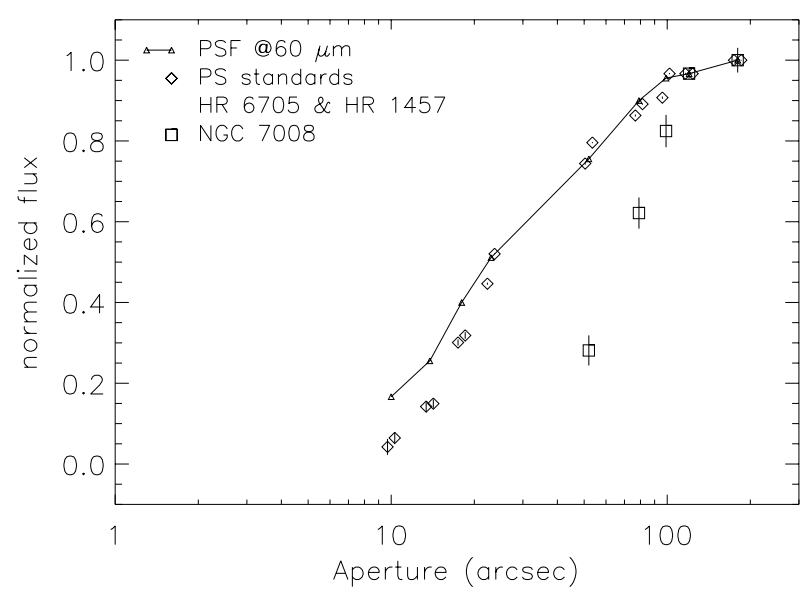

Fig. 14. Multi-aperture profile of NGC 7008 at $60 \mu \mathrm{m}$. The profiles of the theoretical PSF and the point sources HR 6705 and HR 1457 are shown for comparison.

flux ratio $\frac{f_{25}}{f_{60}}$ is smaller for NGC 7008 than for NGC 6543, implying a lower temperature for the long wavelength part. However, the 25,60 and $100 \mu \mathrm{m}$ fluxes cannot be simultaneously fitted by a single modified blackbody with $\beta=1.5$. On the other hand, the higher ratio $\frac{f_{12}}{f_{25}}$ for NGC 7008 suggests a higher temperature. Both constraints can only be fulfilled by the superposition of two blackbodies with temperatures $T_{1}=120 \mathrm{~K}$ and $T_{2}=55 \mathrm{~K}$. The hotter one dominates the $25 \mu \mathrm{m}$ emission, the cooler one the $60 \mu \mathrm{m}$ emission. This is consistent with our finding that the $60 \mu \mathrm{m}$ emission is more extended. Su et al. (2004) found two temperature components determining the SED shape of the PN NGC 2346.

Some authors have emphasized that the IRAS $12 \mu \mathrm{m}$ flux may be heavily contaminated by line emission (e.g. Lenzuni et al. 1989; Stasińska \& Szcerba 1999). However, since the $12 \mu \mathrm{m}$ point is on the Wien branch of the warm blackbody, reducing its temperature by a few $\mathrm{K}$ will still fit the $25 \mu \mathrm{m}$ flux point, but the model will then be significantly below the $12 \mu \mathrm{m}$ flux point, with the excess attributable to line emission. This case is indicated by the dashed-dotted line in Fig. 16, where a $110 \mathrm{~K}$ blackbody can give a $40 \%$ flux reduction at $12 \mu \mathrm{m}$ but reproduces the $25 \mu \mathrm{m}$ emission well.
For two sources in their sample Bowey et al. (2002) found a significant difference between the forsterite $69 \mu \mathrm{m}$ peak temperatures $(\approx 130 \mathrm{~K})$ and the continuum temperatures $(\approx 60 \mathrm{~K})$. An emissivity index of $\beta=0$, implying large grains, could produce agreement between the forsterite and continuum temperatures, however the authors prefered the explanation that the forsterite and continuum emitting grains are spatially separated. A similar condition may prevail in NGC 7008 with two dust populations of different properties.

We use a distance of $1.0 \mathrm{kpc}$, averaging values of $0.8 \mathrm{kpc}$ published by Daub (1982), $1.1 \mathrm{kpc}$ by Pottasch (1983), $0.9 \mathrm{kpc}$ by Maciel (1984), $0.9 \mathrm{kpc}$ by Cahn et al. (1992) and $1.3 \mathrm{kpc}$ by Zhang (1995). However Ciardullo et al. (1999) derived a distance of only $370 \mathrm{pc}$ by deriving a direct distance from the brightness of a close main sequence companion detected in a HST snapshot survey. According to the formula of Eq. (1) and with the same assumptions for the dust opacity $\kappa_{850 \mu \mathrm{m}}(\beta=1.5)$ as used for NGC 6543 (see Sect. 2.5), and $S_{850 \mu \mathrm{m}}=0.073 \mathrm{Jy}$ we derive a dust mass $M_{\text {dust }}=1.2 \times 10^{-3} M_{\odot}$, i.e. about twice as much as in NGC 6543. Lenzuni et al. (1989) obtained $M_{\text {dust }}=8.5 \times 10^{-5} M_{\odot}$ with a distance of $790 \mathrm{pc}$, Pottasch et al. (1984) $M_{\text {dust }}=2.5 \times 10^{-4} M_{\odot}$ with a distance of $1.0 \mathrm{kpc}$.

We calculate the gas mass according to formula (4) in Stasińska \& Szcerba (1999) together with their data in on-line Table 1, in particular using the reddening corrected nebular flux in $\mathrm{H}_{\beta}$. This yields a gas mass $M_{\mathrm{gas}}=6.9 \times 10^{-2} M_{\odot}$ and a dust-togas mass ratio of $\frac{M_{\text {dust }}}{M_{\text {gas }}}=1.7 \times 10^{-2}$, higher than in the interstellar medium of the Milky Way with $\frac{M_{\text {dust }}}{M_{\text {gas }}}{ }_{\text {MilkyWay }}^{\text {average }}=6.25_{-1.71}^{+3.75} \times 10^{-3}$ (Sodroski et al. 1994). Lenzuni et al. (1989) derived a ratio $\frac{M_{\text {dust }}}{M_{\text {gas }}}=5.0 \times 10^{-4}$, Pottasch et al. (1984) $\frac{M_{\text {dust }}}{M_{\text {gas }}}=6.7 \times 10^{-4}$. However, from the data of Pottasch et al. (1984) a gas mass of $M_{\text {gas }}=3.7 \times 10^{-2} M_{\odot}$ is implied, which is only a factor of 2 smaller than ours. It is therefore our revised view of the dust emission which leads to a considerably higher dust mass and dust-to-gas mass ratio. The dust-to-gas mass ratio suggests that dust enrichment occurred in this source.

\section{Summary and conclusions}

The multi-aperture photometry mode is an efficient observing technique for determining whether a particular object has extended emission in a specific size range. It has proved to be a useful exploration tool before executing full mapping in a timelimited situation such as prevails with satellite observations.

We have used multi-aperture mode data products from the ISO Data Archive to search for and investigate extended IR emission in the two PNe NGC 6543 and NGC 7008. This analysis, complemented by ISOPHOT and ISOCAM spectra as well as IRAS and ISOPHOT photometry, led to the following results:

\section{For NGC 6543:}

- the $3.6 \mu \mathrm{m}$ emission of NGC 6543 is extended and well described by a Gaussian with $F W H M=14$ " which coincides with the core region of the optical nebula. The homogeneously distributed emission consists of $65 \%$ continuum emission and $35 \%$ line emission by hydrogen recombination lines of $\operatorname{Br} \alpha, \operatorname{Pf} \gamma$ and $\operatorname{Pf} \delta$. The shape of the continuum suggests that it is a mixture of radiation from the hot central star and radiation from very small dust grains;

- for the $12 \mu \mathrm{m}$ emission of NGC 6543 the profile suggests a more complex morphology: a bright central component with 

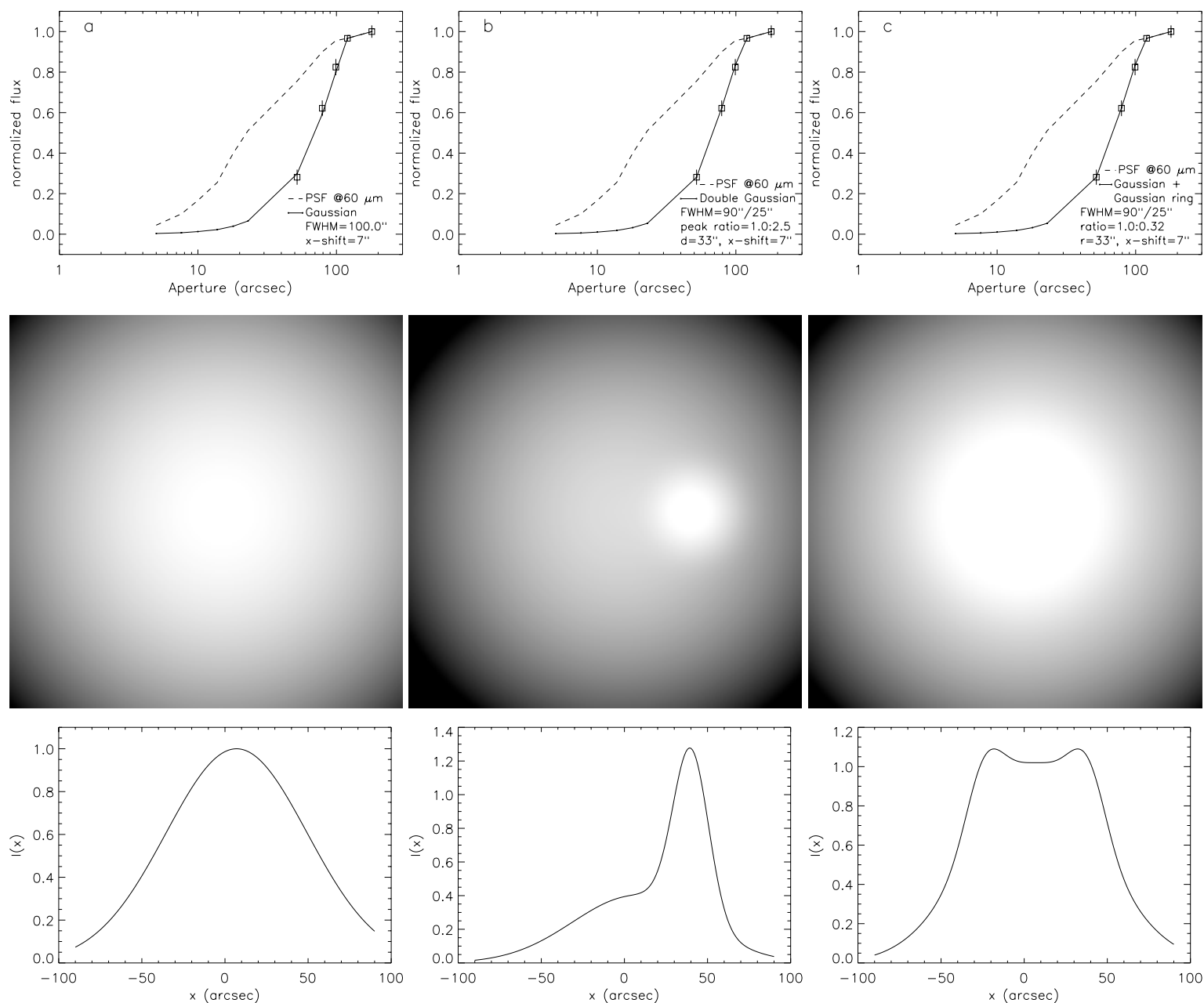

Fig. 15. Modelling of the NGC $700860 \mu \mathrm{m}$ multi-aperture profile. Upper panels: fits of different models to the measured data points; middle panels: grey-scale representation of the modelled intensity distributions - the size of the grey-scale images is $180^{\prime \prime} \times 180^{\prime \prime}$; lower panels: cut through the (normalized) intensity distributions along the $x$-axis. a) Model 1: Gaussian with $F W H M=100^{\prime \prime}$ shifted by 7". b) Model 2: Gaussian with $F W H M=90^{\prime \prime}$ plus offset $\left(d=33^{\prime \prime}\right)$ Gaussian with $F W H M=25^{\prime \prime}$ both shifted by $7^{\prime \prime}$ with regard to the centre; the peak ratio of the two Gaussians is 1:2.5. c) Model 3: central Gaussian with $F W H M=90^{\prime \prime}$ plus offset Gaussian ring $\left(r=33^{\prime \prime}\right)$ with $F W H M=25^{\prime \prime}$ both shifted by 7 " with regard to the centre; the peak ratio of the central Gaussian and the ring is 1:0.32. Details of the model parameters have been compiled in Table 4.

Table 4. Compilation of model parameters for the NGC $700860 \mu \mathrm{m}$ profile (see Figs. 14 and 15). The positional offset indicates that the total intensity distribution has been shifted (arbitrarily in the $+x$-direction) with regard to the origin of the multi-aperture sequence taking into account the offset with regard to the SIMBAD position.

\begin{tabular}{|c|c|c|c|c|c|}
\hline $\begin{array}{c}\text { Model } \\
\#\end{array}$ & Component 1 & $\begin{array}{l}\text { Parameter } \\
\text { and range }\end{array}$ & Component 2 & $\begin{array}{l}\text { Parameter } \\
\text { and range }\end{array}$ & $\begin{array}{c}\text { Positional } \\
\text { offset }\end{array}$ \\
\hline 1 & central Gaussian & $F W H M=100^{\prime \prime} \pm 10^{\prime \prime}$ & - & - & $7^{\prime \prime}$ \\
\hline 2 & central Gaussian & $\begin{array}{l}F W H M=90^{\prime \prime+32^{\prime \prime}}-18^{\prime \prime} \\
I_{\text {rel }}=1.0\end{array}$ & offset Gaussian & $\begin{array}{l}F W H M=25^{\prime \prime} \pm 20^{\prime \prime} \\
d=33^{\prime \prime} 0 \pm 4^{\prime \prime} 0 \\
I_{\text {rel }}=2.5_{-2.47}^{+4.5}\end{array}$ & $7^{\prime \prime}$ \\
\hline 3 & central Gaussian & $\begin{array}{l}F W H M=90^{\prime \prime+32^{\prime \prime}}-18^{\prime \prime} \\
I_{\text {rel }}=1.0\end{array}$ & Gaussian ring & $\begin{array}{l}F W H M=25^{\prime \prime} \pm 20^{\prime \prime} \\
r=33^{\prime \prime} 0 \pm 4^{\prime \prime} 0 \\
I_{\text {rel }}=0.323_{-0.319}^{+0.581}\end{array}$ & $7^{\prime \prime}$ \\
\hline
\end{tabular}

a FWHM of $4^{\prime \prime}$ is surrounded by a second component of similar width at a distance of 7" with an integrated relative intensity of $60 \%$ of the central one. A third component with a relative intensity of $10 \%$ is located at a distance of $17^{\prime \prime}$;

- the $12 \mu \mathrm{m}$ multi-aperture analysis was the only one which could be checked with a direct image constructed from an ISOCAM-CVF scan which was convolved with the
ISOPHOT $12 \mu \mathrm{m}$ bandpass response. This direct comparison confirms that a compact double source with a relative distance of 7 " determines the inner structure of the profile; - the double peak can be explained by strong line emission of [ArIII] $8.99 \mu \mathrm{m}$ and [SIV] $10.51 \mu \mathrm{m}$;

- at $60 \mu \mathrm{m}$ NGC 6543 could not be resolved down to the diffraction limited aperture size of 52" . A detailed ISOPHOT 
Table 5. Photometry of NGC 7008 from IRAS (Point Source Catalog, PSC, Small Scale Structure Catalog, X, and Chopped Photometric Channel, CPC, from Leene \& Pottasch 1988) and ISOPHOT multi-aperture (ma) photometry. Fluxes are not color corrected. The ratio $\frac{I_{120}}{I_{180}}$ is the essential model parameter for the flux reconstruction. For the double Gaussian case the fraction of flux associated with both components is given.

\begin{tabular}{|c|c|c|c|c|c|c|c|c|c|c|c|}
\hline \multirow{4}{*}{$\begin{array}{c}\lambda_{\mathrm{c}} \\
(\mu \mathrm{m})\end{array}$} & \multicolumn{4}{|c|}{ IRAS } & \multicolumn{7}{|c|}{ ISOPHOT ma photometry } \\
\hline & \multirow{3}{*}{$\begin{array}{c}\text { PSC20590+5420 } \\
\text { flux } \\
\text { (Jy) }\end{array}$} & \multicolumn{2}{|c|}{$X 20590+543$} & \multirow{3}{*}{$\begin{array}{c}\text { CPC } 9 \times 9 \\
\text { flux } \\
(\mathrm{Jy})\end{array}$} & \multirow{3}{*}{$\begin{array}{l}180^{\prime \prime} \\
\text { flux } \\
(\mathrm{Jy})\end{array}$} & \multicolumn{2}{|c|}{ Gaussian profile } & \multicolumn{4}{|c|}{ Double Gaussian } \\
\hline & & flux & size & & & $\frac{I_{120}}{I_{180}}$ & flux & $\frac{I_{120}}{I_{180}}$ & flux & comp1 & comp2 \\
\hline & & (Jy) & $\left({ }^{\prime}\right)$ & & & & (Jy) & & (Jy) & $(\%)$ & $(\%)$ \\
\hline 12 & 1.5 & 4 & 1.3 & & - & - & - & - & - & - & - \\
\hline 25 & 24.5 & 41 & 1.1 & - & $35.2 \pm 0.7$ & $0.963_{-0.006}^{+0.007}$ & $36.3 \pm 0.8$ & $0.985_{-0.001}^{+0.001}$ & $34.8 \pm 0.7$ & 88 & 12 \\
\hline $60^{*}$ & 44.3 & 60 & 1.7 & $53-69$ & $40.1 \pm 1.6$ & $0.705_{-0.04}^{+0.005}$ & $85.4 \pm 6.8$ & $0.795_{-0.014}^{+0.0113}$ & $61.5 \pm 2.7$ & 84 & 16 \\
\hline 100 & 35.4 & - & - & $26-44$ & - & - & - & - & - & - & - \\
\hline
\end{tabular}

* $50 \mu \mathrm{m}$ for IRAS CPC.

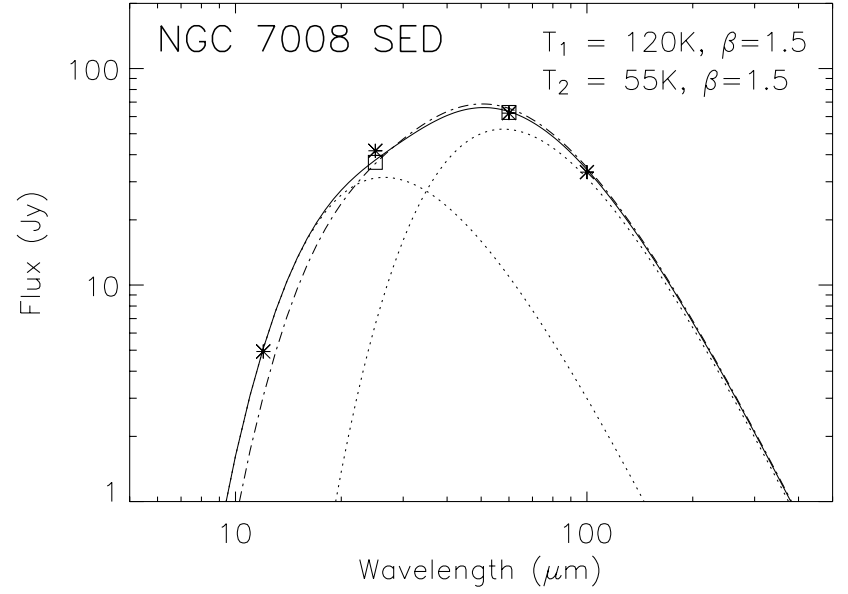

Fig. 16. Spectral energy distribution of NGC 7008 from ISOPHOT multi-aperture photometry (flux reconstruction, assuming double Gaussian, squares). Asterisks indicate IRAS Small Scale Structure Catalog and Chopped Photometric Channel photometry. Fluxes are compiled in Table 5. They have been color corrected according to the dominating modified blackbody emission in the band. Photometric accuracies are about $10 \%$. The solid line represents the superposition of the emission of the two modified blackbodies with the indicated parameters, the dashed lines represent the contributions by the individual ones. The dashed-dotted line represents the corresponding superposition of a $110 \mathrm{~K}$ and $55 \mathrm{~K}$ modified blackbody allowing for a significant contribution of emission lines to the $12 \mu \mathrm{m}$ band.

$3-200 \mu \mathrm{m}$ SED suggests that the $60 \mu \mathrm{m}$ flux is thermal dust continuum emission characterized by a modified blackbody with a temperature of $85 \mathrm{~K}$ and an emissivity index of $\beta=1.5$. The dust, with $M_{\text {dust }}=6.4 \times 10^{-4} M_{\odot}$, seems to be well mixed with the ionised gas of the core region. We derive a dust-to-gas mass ratio of $4.6 \times 10^{-3}$.

\section{For NGC 7008:}

- at $25 \mu \mathrm{m}$ the central component of NGC 7008 is extended, with a $F W H M$ of about $50^{\prime \prime}$. On top of that is a ring- or spotlike structure at a radius of $35^{\prime \prime}$ which corresponds to the rim of the optical nebula;

- the $60 \mu \mathrm{m}$ profile of NGC7008 is more extended with a $F W H M$ of $90^{\prime \prime}$ and the same additional ring-or spot-like structure at a radius of $33^{\prime \prime}$ which results in the emission peaking in this ring;

- the morphological difference in the 25 and $60 \mu \mathrm{m}$ filters suggests that the $60 \mu \mathrm{m}$ measurement traces colder dust which reaches farther out. Analysis of the SED with limited data points might indeed suggest that there are two main temperature components, the warmer one, dominating the central $25 \mu \mathrm{m}$ emission, with a temperature of $120 \mathrm{~K}$, and the colder one, dominating the outer $60 \mu \mathrm{m}$ emission, with a temperature of $55 \mathrm{~K}$. For the latter component a mass of $1.2 \times 10^{-3} M_{\odot}$ is derived, which is significantly higher than found previously. We also derive a dust-to-gas mass ratio of $1.7 \times 10^{-2}$.

The ISOCAM image of NGC 6543 shows that this PN is a complex gas-filled structure, and one of the emission peaks in each image of Fig. 7 is located at the stellar position, as identified from the SIMBAD coordinates, so strong emission lines should be expected there due to the Of $+\mathrm{WR}$ star. The estimates of $\frac{M_{\text {gas }}}{M_{\text {dust }}}$ for NGC 6543 of around 190 to 260, and for NGC 7008 of around 59, suggests that the gas-to-dust ratio in NGC 7008 is significantly lower than the average value of 160 for the interstellar medium which hints at dust enrichment by this source.

From their statistical analysis of dust temperatures, Lenzuni et al. (1989) found a systematic decrease with the nebula radius. This implied also a decrease of the dust mass with the nebula radius and an increase of the number of dust grains which was ascribed to significant evolution and processing of the grains in the nebula environment. We cannot revise their statistical analysis on the basis of two objects, however, our results for NGC 6543 and NGC 7008 and the result by Su et al. (2004) for NGC 2346 show that their approach was too simplified: the grain emission in PNe is not always characterised by one single temperature, and low dust temperatures increase the dust mass considerably. Therefore more detailed spectral and spatial data are necessary when attempting to find evolutionary signatures.

In the on-line Appendix A we present the general analysis and interpretation of the multi-aperture observations with ISOPHOT, implemented via the Astronomical Observation Template (AOT) PHT04:

- We have developed a method for consistent background scaling and subtraction in ISOPHOT multi-aperture observations.

- We present a revised method of uncertainty calculation with regard to the one given in the ISOPHOT Handbook (Laureijs et al. 2003). This takes the source-to-background contrast into account and thus allows a better handle in the assessment of sufficient $\mathrm{S} / \mathrm{N}$ and hence the reliability of a given multi-aperture profile.

- We describe a method how to correct for signal transients by fitting the signal time series with combinations of exponential functions and adapting their time constants to the curvature of the measured signals. We demonstrate the improvements for the $12 \mu \mathrm{m}$ multi-aperture profile of NGC 6543 . 
- By means of normalized multi-aperture profiles a direct comparison with point source and flat-sky references is possible. Several such references are available from in-flight calibration measurements and an overview of those has been given here.

- We have given recipes to interprete the resulting source profiles more quantitatively by means of simple model images, including Gaussians, multiple PSFs and ring structures. They determine the radii at which source emission maxima are located.

- The method to obtain absolute flux levels for these measurements where the calibration was not established in the $180^{\prime \prime}$ aperture has been described and its accuracy has been verified against photometric standard stars.

- Given a source profile which extends into the annulus used for background subtraction, we have described a method to reconstruct the total source flux.

- We have also outlined the limitations of this mode.

The data analysis method described in Sect. A.1.2 has been implemented in the ISOPHOT data reduction pipeline (OLP) and data products of this observing mode in the ISO Data Archive contain all the relevant information to produce multi-aperture profiles for immediate interpretation with the methods outlined in this paper. In the case of transient flags being set, it is advisable to perform an interactive re-analysis of the signal time series as outlined in Sect. A.1.4.

Acknowledgements. The ISOPHOT Data Centre at MPIA is supported by Deutsches Zentrum für Luft- und Raumfahrt e.V. (DLR) with funds of Bundesministerium für Bildung und Forschung, grant No. 50 QI 0201. It is a pleasure for us to thank our former colleagues of the ISOPHOT Instrument Dedicated Team in Villafranca and the ISOPHOT Data Centre in Heidelberg for their extreme engagement in the commissioning and scientific validation of the observing modes and the calibration of the instrument. We thank the referee Dr. R. J. Laureijs for valuable and constructive comments.

\section{References}

Acosta-Pulido, J., Gabriel, C., \& Castañeda, H., Experimental Astronomy, 10, 333

Balick, B. 2004, AJ, 127, 2262

Balick, B., Gonzalez, G., Frank, A., \& Jacoby, G. J. 1992, ApJ, 392, 582

Balick, B., Wilson, J., \& Hajian, A. R. 2001, AJ, 121, 354

Bianchi, L., Cerrato, S., \& Grewing, M. 1986, A\&A, 169, 227

Blommaert, J., Siebenmorgen, R., Coulais, A., et al. 2003, The ISO Handbook, II, CAM - The ISO Camera, ESA SP-1262
Boulanger, F., Lorente, R., Miville Deschênes, M. A., et al. 2005, A\&A, 436, 1151

Bowey, J. E., Barlow, M. J., Molster, F. J., et al. 2002, MNRAS, 331, L1

Cahn, J. H., Kaler, J. B., \& Stanghellini, L. 1992, A\&AS, 94, 399

Ciardullo, R., Bond, H. E., Sipior, M. S., et al. 1999, AJ, 118, 488

Cohen, M. 2003, Proc. of the Conference The calibration legacy of the ISO mission, Villafranca, 05-09 February 2001, ESA SP-481, 135

Daub, C. T. 1982, ApJ, 260, 612

Gabriel, C., Acosta-Pulido, J., Heinrichsen, I., Morris, H., \& Tai, W. M. 1997, The ISOPHOT Interactive Analysis PIA, a Calibration and Scientific Analysis Tool, in ADASS VI., ed. G. Hunt, \& H. E. Payne, ASP Conf. Ser., 125, 108 Górny, S. K., Stasińska, G., \& Tylenda, R. 1997, A\&A, 318, 256

Gry, C., Swinyard, B., Harwood, A., et al. 2003, The ISO Handbook, III, LWS The Long Wavelength Spectrometer, ESA SP-1262

Hammersley, P. L., \& Jourdain de Muizon, M. 2003, Proc. of the Conference The calibration legacy of the ISO mission, Villafranca, 05-09 February 2001, ESA SP-481, 129

Hora, J. L., Latter, W. B., Allen, L. E., et al. 2004, ApJS, 154, 296

Hua, C. T., \& Louise, R. 1972, A\&A, 21, 193

Jewitt, D. C., Danielson, G. E., \& Kupferman, P. N. 1986, ApJ, 302, 727

Kessler, M. F., Steinz, J. A., Anderegg, M. E., et al. 1996, A\&A, 315, L27

Kessler, M. F., Müller, T., Leech, K., et al. 2003, The ISO Handbook, I, ISO Mission \& Satellite Overview, ESA SP-1262

Klaas, U., Haas, M., Müller, S. A. H., et al. 2001, A\&A, 379, 823

Kwok, S. 2001, in Planetary Nebulae: Their Evolution and Role in the Universe, Proc. 209th Symp. IAU, Canberra, Australia, ed. Kwok et al., 3

Kwok, S. 2004, Nature, 430, 985

Laureijs, R. J., Klaas, U., Richards, P. J., \& Schulz, B., \& Ábrahám, P. 2003, The ISO Handbook, IV, PHT - The Imaging Photo-Polarimeter, ESA SP-1262

Leene, A., \& Pottasch, S. R. 1988, A\&A, 202, 203

Leinert, Ch., Ábrahám, P., Acosta-Pulido, J., Lemke, D., \& Siebenmorgen, R. 2002, A\&A, 393, 1073

Lemke, D., Klaas, U., Abolins, J., et al. 1996, A\&A, 315, L64

Lenzuni, P., Natta, A., \& Panagia, N., et al. 1989, ApJ, 345, 306

Maciel, W. J. 1984, A\&AS, 55, 253

Müller, T. G. 1999, Proc. of the Workshop ISO Beyond Point Sources: Studies of Extended Infrared Emission, Villafranca, 14-17 September 1999, ESA SP455,33

Müller, T. G. 1999, Proc. of the Workshop ISO Beyond Point Sources: Studies of Extended Infrared Emission, Villafranca, 14-17 September 1999, ESA SP455, 41

Persi, P., Cesarsky, D., Marenzi, A. R., et al. 1999, A\&A, 351, 201

Pottasch, S. R. 1983, Proc. IAU Symp., 103, Planetary Nebulae, London, 9-13 August 1983, 103

Pottasch, S. R., Baud, B., Beintema, D., et al. 1984, A\&A, 138, 10

Reimers, C., Dorfi, E. A., \& Höfner, S. 2000, Ap\&SS, 272, 205

Schroeder, D. J. 1987, Astronomical Optics (San Diego: Academic Press)

Siebenmorgen, R., Prusti, T., Natta, A., \& Müller, T. G. 2000, A\&A, 361, 258

Sodroski, T. J., Bennett, C., Boggess, N., et al. 1994, ApJ, 428, 638

Stasińska, G., \& Szcerba, R. 1999, A\&A, 352, 297

Su, K. Y. L., Kelly, D. M., Latter, W. B., et al. 2004, ApJS, 154, 302

Tajitsu, A., \& Tamura, S. 1998, AJ, 115, 1989

Zhang, C. Y. 1995, ApJS, 98, 659 
U. Klaas et al.: ISOPHOT multi-aperture photometry. I., Online Material $p 1$

\section{Online Material}




\section{Appendix A: The technique and calibration of ISOPHOT multi-aperture photometry}

The ISOPHOT instrument (Lemke et al. 1996) onboard the Infrared Space Observatory ISO (Kessler et al. 1996) contained, as one subinstrument, the aperture photometer ISOPHOT-P. Three single detectors, covering the wavelength ranges 3.3-15 $\mu \mathrm{m}$ (detector P1), 20-25 $\mu \mathrm{m}$ (P2) and 60-100 $\mu \mathrm{m}(\mathrm{P} 3)$, respectively, could be used in combination with 11 circular apertures with a diameter between $5^{\prime \prime}$ and $180^{\prime \prime}$ and two rectangular apertures. One observing mode was multiaperture photometry (Astronomical Observation Template, AOT PHT04) which, for a single selected photometric filter, switches through a set of circular apertures on a target to assess the growth of flux with radius; for details please refer to the ISOPHOT Handbook (Laureijs et al. 2003).

The original purpose in designing the observing mode was to provide a quicker method of determining whether an object included extended emission than by making a fully sampled map. It took around half the time to make multi-aperture observations as it did to make maps. Because of the integration over circular annuli, multi-aperture photometry is sensititive to detecting faint extended surface brightness around compact sources. One constraint is, however, that the detailed structure and exact location of the peaks in the extended emission cannot be determined.

In this on-line appendix we describe an analysis technique and calibration for the ISOPHOT-P multi-aperture mode, provided the $120^{\prime \prime}$ and $180^{\prime \prime}$ apertures plus at least two smaller ones were selected for the observation. Around sixty objects were observed with this multi-aperture mode of ISOPHOT, including embedded young stellar objects, main sequence stars, evolved stars, and planetary nebulae. An overview of the targets and the observing details is given in Table A.6.

In Sect. A.1 we outline the construction of source profiles from the sequences, discuss the involved uncertainties, describe a method of correcting signal transients and consider the two types of reference source, namely point sources without any extension, and flat extended emission, from the sky background. In Sect. A.2 we describe how to quantitatively interpret the resulting profiles with simple intensity distributions. In Sect. A.3 we discuss and verify the photometric calibration for the $180^{\prime \prime}$ aperture, which is not the standard photometric aperture for the P1 and $\mathrm{P} 2$ detectors, and we describe a method of total flux reconstruction with the help of the intensity model.

\section{A.1. Data analysis method}

\section{A.1.1. Background}

A key element in the analysis of aperture photometry measurements is the sky background subtraction. Since the aperture sequence measurements were obtained in staring mode, the observers performed an independent background measurement which was often restricted to the largest aperture in use. This observing strategy turned out to have the following disadvantages:

1) an accurate absolute calibration of ISOPHOT-P could not be established for all apertures in the end. For the the absolute calibration of a multi-aperture sequence, a measurement illuminating the detector with the internal calibration source, the FCS, was obtained in the largest aperture of the sequence, usually the $180^{\prime \prime}$ one, in order to derive the actual detector responsivity. In particular for the filters of the P1 and P2 detectors the illumination of the $180^{\prime \prime}$ aperture by the FCS was not calibrated against celestial standards;
2) the uncertainties in the signal determination of the FCS measurements associated with the independent source and the separate background measurement could lead to some mismatch in the absolute flux calibration between both measurements;

3) due to non-flat aperture responses the background contribution did not scale like the geometrical aperture sizes.

In order to overcome disadvantage \#2 Müller (1999b) proposed determining the background directly from the measurement sequence, namely from the annulus between the $120^{\prime \prime}$ and $180^{\prime \prime}$ aperture borders. This method was first applied to multi-aperture sequences on Herbig Ae/Be stars which included a direct comparison with 2D images (Siebenmorgen et al. 2000). This procedure may suggest that the source should not be more extended than $2^{\prime}$ in diameter. However, as will be shown below, this works even if part of the source is located inside this annulus. Nevertheless the source should not be more extended than $3^{\prime}$ in diameter, the size of the largest aperture, which would be also a limitation for the conventional measurement and evaluation strategy. The background subtraction method outlined by Müller (1999b) was eventually implemented, with some slight modifications, into the ISOPHOT Offline Processing Pipeline OLP V10.0 (Laureijs et al. 2003).

\section{A.1.2. Construction of normalised source profiles}

The analysis is done on the signal level (in units of $\mathrm{V} \mathrm{s}^{-1}$ ), the relation to the absolute flux density is shown in Sect. A.3.1. However, we will use signal ratios in the end, and since the absolute flux scaling cancels out, the signal ratios are equivalent to flux ratios. Hence the signal ratios can be called "normalized fluxes".

The total signal received in any aperture is the sum of the contributions by the source and the background,

$S_{\text {aper }}=S_{\text {aper }}^{\text {source }}+S_{\text {aper }}^{\text {bg }}$.

The background is most reliably determined from the signals in the two largest apertures, since there its contribution is largest. The background signal scales with the area of the aperture. This can be written as a product of the measured background per area $\mathrm{bg}_{\text {per area }}$ with the aperture area:

$S_{\text {aper }}^{\mathrm{bg}}=\mathrm{bg}_{\text {per area }} \cdot A_{\text {aper }} \cdot a_{\text {aper }}^{\text {corr }}$

with $A_{\text {aper }}$ being the nominal aperture area as given in Laureijs et al. (2003), Appendix A.1.2. Due to non-flatness of the apertures, for examples see Laureijs et al. (2003), Fig. 4.9 in Sect. 4.5.1.2, a non-linear scaling between different apertures, $a_{\text {aper }}^{\text {corr }}$, has to be introduced (Müller 1999a).

As a reference and for the purpose of the calibration we assume that the source is a point source and its signal in each aperture scales with the fraction of the Point Spread Function (PSF) contained in the aperture, $f_{\text {aper }}^{\mathrm{psf}}$ :

$S_{\text {aper }}^{\text {source }}=S_{\text {tot }}^{\text {source }} \cdot f_{\text {aper }}^{\text {psf }}$.

The values of $f_{\mathrm{aper}}^{\mathrm{psf}}$ are tabulated in Laureijs et al. (2003), Table 2.3. Hence, from Eqs. (A.1)-(A.3),

$S_{\text {tot }}^{\text {source }}=\frac{S_{\text {aper }}-\mathrm{bg}_{\text {per area }} \cdot A_{\text {aper }} \cdot a_{\text {aper }}^{\text {corr }}}{f_{\text {aper }}^{\text {psf }}}$. 
Table A.1. Empirical aperture correction factors $a_{\text {aper }}^{\text {corr }}$ for scaling the background level. The method for determining these factors and its associated uncertainties is described in the text. Values marked by an "e" are adopted from measurements in larger apertures - for small apertures the background contribution is mostly negligible. Values in italics are updates with regard to the ones in Table 7.2 in the ISOPHOT Handbook (Laureijs et al. 2003). For those filters with all values set to 1.00 no correction could be derived, see text for a discussion of the relevance.

\begin{tabular}{cccccccccccc}
\hline \hline \multirow{2}{*}{$\begin{array}{c}\text { Filter } \\
\lambda_{\mathrm{c}}\end{array}$} & 5 & 7.6 & 10 & 13.8 & 18 & 23 & 52 & 79 & 99 & 120 & 180 \\
$(\mu \mathrm{m})$ & & & & \multicolumn{10}{c}{ Correction factors for aperture } \\
\hline 3.3 & 1.00 & 1.00 & 1.00 & 1.00 & 1.00 & 1.00 & 1.00 & 1.00 & 1.00 & 1.00 & 1.00 \\
3.6 & 1.00 & 1.00 & 1.00 & 1.00 & 1.00 & 1.00 & 1.00 & 1.00 & 1.00 & 1.00 & 1.00 \\
4.8 & 1.00 & 1.00 & 1.00 & 1.00 & 1.00 & 1.00 & 1.00 & 1.00 & 1.00 & 1.00 & 1.00 \\
7.3 & 0.50 & 0.50 & 0.50 & 0.50 & 0.50 & 0.50 & 0.50 & 0.50 & 0.65 & 0.80 & 1.00 \\
7.7 & 1.00 & 1.00 & 1.00 & 1.00 & 1.00 & 1.00 & 1.00 & 1.00 & 1.00 & 1.00 & 1.00 \\
10 & 0.40 & 0.40 & 0.40 & 0.40 & 0.40 & 0.40 & 0.40 & 0.40 & 0.50 & 0.65 & 1.00 \\
11.3 & 1.00 & 1.00 & 1.00 & 1.00 & 1.00 & 1.00 & 1.00 & 1.00 & 1.00 & 1.00 & 1.00 \\
12 & $0.90 \mathrm{e}$ & 0.90 & 0.90 & 0.75 & 0.75 & 0.80 & 0.62 & 0.68 & 0.70 & 0.78 & 1.00 \\
12.8 & 1.00 & 1.00 & 1.00 & 1.00 & 1.00 & 1.00 & 1.00 & 1.00 & 1.00 & 1.00 & 1.00 \\
15 & $0.35 \mathrm{e}$ & $0.35 \mathrm{e}$ & 0.35 & 0.35 & 0.35 & 0.35 & 0.55 & 0.62 & 0.63 & 0.70 & 1.00 \\
20 & $0.40 \mathrm{e}$ & $0.40 \mathrm{e}$ & 0.40 & 0.40 & 0.40 & 0.65 & 1.00 & 1.08 & 1.04 & 1.00 & 1.00 \\
25 & $0.45 \mathrm{e}$ & $0.45 \mathrm{e}$ & $0.45 \mathrm{e}$ & $0.45 \mathrm{e}$ & $0.45 \mathrm{e}$ & 0.45 & 0.48 & 0.80 & 0.91 & 0.97 & 1.00 \\
60 & $0.30 \mathrm{e}$ & $0.30 \mathrm{e}$ & 0.30 & 0.30 & 0.40 & 0.40 & 2.30 & 1.75 & 1.17 & 1.08 & 1.00 \\
100 & 1.00 & 1.00 & 1.00 & 1.00 & 1.00 & 1.00 & 1.00 & 1.00 & 1.00 & 1.00 & 1.00 \\
\hline
\end{tabular}

For the purpose of the calculation we assume that the increase in source signal from the $120^{\prime \prime}$ to the $180^{\prime \prime}$ aperture is negligible and most of the source intensity is located inside the $120^{\prime \prime}$ aperture diameter (we will later show that this condition needs not to be strictly fulfilled). Hence, from the measurements of the total signals in the $120^{\prime \prime}$ and $180^{\prime \prime}$ apertures, $S_{120}$ and $S_{180}$, and with $a_{180}^{\text {corr }}=1.0$ the background per area is

$\mathrm{bg}_{\text {per area }}=\frac{S_{180}-S_{120} \cdot \frac{f_{180}^{\mathrm{psf}}}{f_{120}^{\mathrm{psf}}}}{A_{180}-A_{120} \cdot a_{120}^{\mathrm{corr}} \cdot \frac{f_{180}^{\mathrm{psf}}}{f_{120}^{\mathrm{psf}}}}$.

The background signal for each aperture can then be calculated according to Eq. (A.2) and the source signal for each aperture is derived by solving Eq. (A.1).

The signals are normalised relative to the $180^{\prime \prime}$ aperture in order to enable straightforward comparison of profiles of different sources, in particular with those of the point source standards,

$S_{\text {aper }}^{\text {norm }}=\frac{S_{\text {aper }}^{\text {source }}}{S_{180}^{\text {source }}}$.

It should be noted that the normalisation and the procedure to derive the background obviously yield a value of 1.0 for the $180^{\prime \prime}$ aperture point, and the $120^{\prime \prime}$ aperture point is always fixed at $\frac{f_{120}}{f_{180}}$, which is only a wavelength dependent ratio.

In case of a point source, according to the definition given in Eq. (A.3), the normalised signal is given by the ratio of the PSF fractions for all apertures.

PHT04 observations listed in Table A.6 were processed using this method implemented as an algorithm in OLP V10.0. The normalised signals are compiled in the headers of the product types PPAP and PPAE under the keyword "NRMFLX". They are accessible via the ISO Data Archive, http: //www . iso.vilspa. esa. es (Kessler et al. 2003).

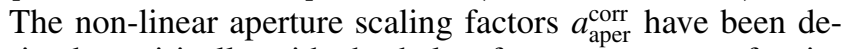
termined empirically with the help of measurements of point source standards for the 12, 20, 25 and $60 \mu \mathrm{m}$ filters. Following the procedure above, normalised profiles have been derived and the aperture scaling factors were iterated until the resulting profiles followed as much as possible the theoretical one (for a verification see Fig. A.5). For those filters with two point source standards the uncertainty in the correction factor can be estimated from the variation of the optimal value for each individual source. It amounts between $3 \%$ and $15 \%$. The impact of this uncertainty is largest for the $120^{\prime \prime}$ aperture, because it affects the determination of the background. For smaller apertures this uncertainty gets less and less important, even if it is relatively large, because the background contribution becomes more and more negligible. The point source standard for the $3.6 \mu \mathrm{m}$ filter is so bright and the background, due to a minimum in the Zodiacal Light, is so faint, that the background subtraction is irrelevant and therefore no correction factors could be determined. In general, due to the faintness of the Zodiacal Light background in the 3 to $5 \mu \mathrm{m}$ range and a steep rise only thereafter (Leinert et al. 2002 ), the $3.3 \mu \mathrm{m}, 3.6 \mu \mathrm{m}$, and $4.8 \mu \mathrm{m}$ aperture sequences are not much affected by unknown aperture correction factors.

For the $7.3 \mu \mathrm{m}$ filter the point source standard turned out to be not reliable, see the discussion in Sect. A.1.5. For this filter and the 10 and $15 \mu \mathrm{m}$ filters the correction factors were tuned so that all available measurements showed consistent profiles; if possible information from other filters, in particular the ones with reliable point source standards, was taken into account. For these filters the uncertainties in $a_{\text {aper }}^{\text {corr }}$ are consequently somewhat larger.

Measurement sequences of point source standards are sometimes not available for the smallest apertures. In these cases the values were set to the value of the smallest measured aperture. The uncertainty introduced by that is quite small, because the background contribution in these apertures is negligible due to the small areas.

Table A.1 lists the values of the correction factor $a_{\text {aper }}^{\text {corr }}$ for all filters. Note that updates have been included with regard to the version contained in Table 7.2 in the ISOPHOT Handbook 
(Laureijs et al. 2003) as a result of the interactive processing we describe in Sects. A.1.4, A.1.5, and A.1.6. For the $7.7 \mu \mathrm{m}$, $11.3 \mu \mathrm{m}, 12.8 \mu \mathrm{m}$, and $100 \mu \mathrm{m}$ filters no correction factors could be derived because of lack of suitable point source reference

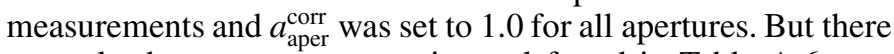
are only three measurements in total found in Table A.6, one each in the latter three filters. For those filters it is advisable to apply the correction factors of adjacent filters as a first order refined correction.

\section{A.1.3. Uncertainties of source profiles}

In order to estimate the impact of various factors on the uncertainty we re-write Eq. (A.6) by applying first Eq. (A.1) and then Eq. (A.2) to its numerator and replacing $b_{\text {perarea }}=\frac{S_{180}^{\mathrm{bg}}}{A_{180}}$. This gives

$S_{\text {aper }}^{\text {norm }}=\frac{S_{\text {aper }}}{S_{180}^{\text {Source }}}-\frac{A_{\text {aper }}}{A_{180}} \cdot \frac{a_{\text {aper }}^{\text {corr }}}{\frac{S_{180}^{\text {sourc }}}{S_{180}^{\text {bg }}}}$.

In the case that the source is very bright with regard to the background, i.e. $\frac{S_{180}^{\text {sourc }}}{S_{180}^{\text {bg }}} \gg 1$, then

$S_{\text {aper }}^{\text {norm }} \approx \frac{S_{\text {aper }}}{S_{180}}$

and $\Delta S_{\text {aper }}^{\text {norm }}$ is determined from the uncertainties of $\Delta S_{\text {aper }}$ and $\Delta S_{180}$ only.

In the case that the source-to-background contrast $\frac{S_{180}^{\text {source }}}{S_{180}^{\text {bg }}} \ll 1$ and hence $S_{180}^{\text {source }} \ll S_{180}$, the first term of Eq. (A.7) can be re-written as

$$
\begin{aligned}
\frac{S_{\mathrm{aper}}}{S_{180}^{\text {source }}} & =\frac{S_{\mathrm{aper}}}{S_{180}^{\mathrm{bg}} \cdot \frac{S_{180 \mathrm{cou}}^{\text {sour }}}{S_{180}^{\mathrm{bg}}}}=\frac{S_{\mathrm{aper}}}{\left(S_{180}-S_{180}^{\text {source }}\right) \cdot \frac{S_{180}^{\text {source }}}{S_{180}^{\text {bg }}}} \\
& \approx \frac{S_{\mathrm{aper}}}{S_{180} \cdot \frac{S_{180}^{\text {source }}}{S_{180}^{\mathrm{bg}}}}
\end{aligned}
$$

giving

$S_{\text {aper }}^{\text {norm }} \approx \frac{S_{180}^{\text {bg }}}{S_{180}^{\text {source }}} \cdot\left(\frac{S_{\text {aper }}}{S_{180}}-a_{\text {aper }}^{\text {corr }} \cdot \frac{A_{\text {aper }}}{A_{180}}\right)$.

As a rule of thumb one should become cautious about the reliability of the derived profiles, once the second term gets in the order of 1 . This is reached for different apertures, if $\frac{S_{180}^{\text {source }}}{S_{180}^{\text {bg }}} \approx \frac{A_{\text {aper }}}{A_{180}}$. For the $99^{\prime \prime}$ aperture $\frac{A_{99}}{A_{180}}=0.3$, for the $79^{\prime \prime}$ aperture $\frac{A_{79}}{A_{180}}=0.19$, for the $52^{\prime \prime}$ aperture $\frac{A_{52}}{A_{180}}=0.09$, for the $23^{\prime \prime}$ aperture $\frac{A_{23}}{A_{180}}=0.02$, and for the $5^{\prime \prime}$ aperture $\frac{A_{5}}{A_{180}}=0.001$. Therefore, profiles with $\frac{S_{180}^{\text {source }}}{S_{180}^{\text {bg }}}<20 \%$ can become quite uncertain. This is backed by the consideration that in the case of $\frac{S_{180}^{\text {soure }}}{S_{180}^{\text {bg }}} \approx 0.1-0.2$ an uncertainty in the background signal determination of 10-20\% corresponds to $100 \%$ of the source signal. However, the exact limit for each individual sequence depends on the accuracy of measuring $S_{\text {aper }}$ for the source signal in the respective aperture, $S_{180}$ for the normalisation, and $S_{120}$ and $S_{180}$ for the determination of $\mathrm{bg}_{\text {perarea }}$. For the ISO Data Archive Products this contrast dependence of the final normalised source signal uncertainty has not been considered in the uncertainty calculation. Based on Eqs. (A.6), (A.1), (A.2) and (A.5) with $R_{\mathrm{psf}}=\frac{f_{\mathrm{psf}}^{180}}{f_{\mathrm{psf}}^{120}}$

$$
\begin{array}{r}
S_{\text {aper }}^{\text {norm }}=\frac{S_{\text {aper }}^{\text {source }}}{S_{180}^{\text {source }}}=\frac{S_{\text {aper }}-S_{\text {aper }}^{\mathrm{bg}}}{S_{180}-S_{180}^{\mathrm{bg}}} \\
S_{\text {aper }}^{\mathrm{bg}}=A_{\text {aper }} \cdot a_{\text {aper }}^{\text {corr }} \cdot \mathrm{bg}_{\text {per area }} \\
\operatorname{bg}_{\text {per area }}=\frac{S_{180}^{\mathrm{bg}}=A_{180} \cdot S_{120} \cdot R_{\mathrm{psf}}}{A_{180}-A_{120} \cdot a_{120}^{\mathrm{corr}} \cdot R_{\mathrm{psf}}}
\end{array}
$$

we derived the following revised uncertainty calculation - with respect to the one described in Sect. 7.9.3 of Laureijs et al. (2003):

$\Delta S_{\text {aper }}^{\text {norm }}=\frac{\sqrt{\Delta S_{\text {aper }}^{2}+\Delta S_{180}^{2}+w_{\mathrm{bg}}^{2} \cdot\left(\Delta S_{120}^{2}+\Delta S_{180}^{2}\right)}}{S_{180}^{\text {source }}}$

$w_{\mathrm{bg}}=\left(1+\frac{A_{\mathrm{aper}}}{A_{180}}\right) \cdot\left(\frac{S_{180}^{\mathrm{bg}}}{S_{180}^{\text {source }}}\right)$.

The factor $w_{\mathrm{bg}}$ guarantees the proper weighting of the uncertainty in the background determination both for the relevant aperture and the $180^{\prime \prime}$ aperture depending on the source-tobackground contrast. Since $S_{120}^{\text {norm }}$ is fixed to $\frac{f_{120}^{\text {psf }}}{f_{180}^{\text {psf }}}$ and $S_{180}^{\text {norm }}=$ 1 , we associate only the simple uncertainties $\frac{\Delta S_{120}}{S_{180}}$ and $\frac{\Delta S_{180}}{S_{180}}$, respectively with them. For the sequences on flat sky backgrounds, which we discuss in Sect. A.1.6, we set $w_{\mathrm{bg}}=2$ to account for the double background subtraction. The term source-to-background contrast is meaningless in this context and drives the uncertainties towards infinity. Since these sequences provide ratios of very small "source" signals the uncertainties should be considered as a lower limit.

Note that the revised uncertainty calculation can be performed from ISO Data Archive products by making use of the SPD products PP1S, PP2S or PP3S, depending on the detector. The header keyword "SRCSIG" contains the source signal $S_{\text {aper }}^{\text {source }}$, and the keyword "BCKSIG" the background signal $S_{\mathrm{aper}}^{\mathrm{bg}}$. The standard uncertainties are contained in the keywords "SCSIGU" and "BKSIGU". "SCSIGU" holds the source signal uncertainty after background subtraction $\Delta s$ aper and "BKSIGU" the uncertainty of the background determination

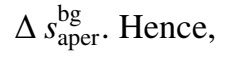

$$
\Delta S_{\text {aper }}=\sqrt{\left(\Delta s_{\text {aper }}^{\text {source }}\right)^{2}-\left(\Delta s_{\mathrm{aper}}^{\mathrm{bg}}\right)^{2}} .
$$

For profiles in filters with $\lambda>5 \mu \mathrm{m}$ and no proper determination of $a_{\text {aper }}^{\text {corr }}$, i.e. all $a_{\text {aper }}^{\text {corr }}=1$, the reliability limit of the source profile has to be set appropriately higher by a factor of 2 .

This error propagation due to statistical uncertainties in signal determination does not include the uncertainty in determining the non-linear aperture scaling factors $a_{\text {aper }}^{\text {corr }}$ and possible systematic uncertainties.

The latter ones may be due to

1) signal transients;

2) non-flat aperture responses. 
The uncertainties due to the non-flat aperture responses cannot be fully characterised currently, in particular for the long wavelength filters (12 to $16 \mu \mathrm{m}$ ) associated with the ISOPHOT-P1 detector. The aperture profiles for these filters are quite asymmetric as shown in Müller (1999a) and in Fig. 4.9 of Sect. 4.5.1.2 in Laureijs et al. (2003). For an extended source this may mean that regions falling onto a particularly sensitive area of the profile will have more weight in the resulting profile. Therefore, aperture sequences in these filters may provide only a qualitative assessment of the parameters used to quantify the source extent.

\section{A.1.4. Signal transient correction}

Signal transients are introduced due to the flux jumps inbetween measurements in the different apertures. Their behaviour depends on the size of the flux jump and the previous history of illumination. There exists no general and consistent transient correction method for the ISOPHOT-P detectors. However, the processing software performs a transient recognition and sets a flag in the resulting data product telling whether signals have stabilized to a final value or whether the signal sequence was still drifting. These processing flags have been made visible more clearly in the ISO Data Archive via the data quality reports. In the compilation of all PHT04 observations with normalised profile processing in Table A.6 we flag those measurements with non-stable signal flagging. When consulting this table whether transients are preferentially associated with shortly exposed measurements, no clear trend can be found. The exposure times per aperture of scientific measurements are $32 \mathrm{~s}, 64 \mathrm{~s}$, or $128 \mathrm{~s}$. Also $128 \mathrm{~s}$ measurements can be affected by transients. It appears that measurements with the ISOPHOT-P3 detector, i.e. $60 \mu \mathrm{m}$ sequences, are less affected than sequences at shorter wavelengths.

In the case of transients it is advisable to perform interactive processing with the ISOPHOT Interactive Analysis software $\left(\mathrm{PIA}^{2}\right)$ to assess the impact of non-stabilised signals more quantitatively. Of course, measurements with longer exposure times can be fitted more reliably.

Our approach described in the following applies for transients of the ISOPHOT-P1, P2 and P3 detectors, as demonstrated in the transient overview in Fig. A.12. As an example we consider and investigate here in detail transients of the ISOPHOT-P1 detector and in particular we derive corrections for the $12 \mu \mathrm{m}$ multi-aperture sequence of NGC 6543, ISO TDT No. 05702402. Figure A.1 displays the temporal suite of signals when switching through the 9 apertures between $10^{\prime \prime}$ and $180^{\prime \prime}$. They were processed with PIA in the same way as the archive data. The archive quality report flags the signals in the $79^{\prime \prime}, 120^{\prime \prime}$, and $180^{\prime \prime}$ apertures as non-stabilised. While the signals for the measurements in the first four apertures are drifting downwards, the signals in the $52^{\prime \prime}$ aperture are quite constant, and for the last four apertures upward signal drifts are observed. The strong upward drift in the $180^{\prime \prime}$ aperture measurement, which is not stabilised in the end, is obvious.

In Fig. A.2a a zoomed view of measurements with transients is displayed. All show more or less strong trends, upwards, downwards or, as in the last case, with a hook response. We

${ }^{2}$ PIA is a joint development by the ESA Astrophysics Division and the ISOPHOT consortium led by the Max-Planck-Institut für Astronomie, Heidelberg.

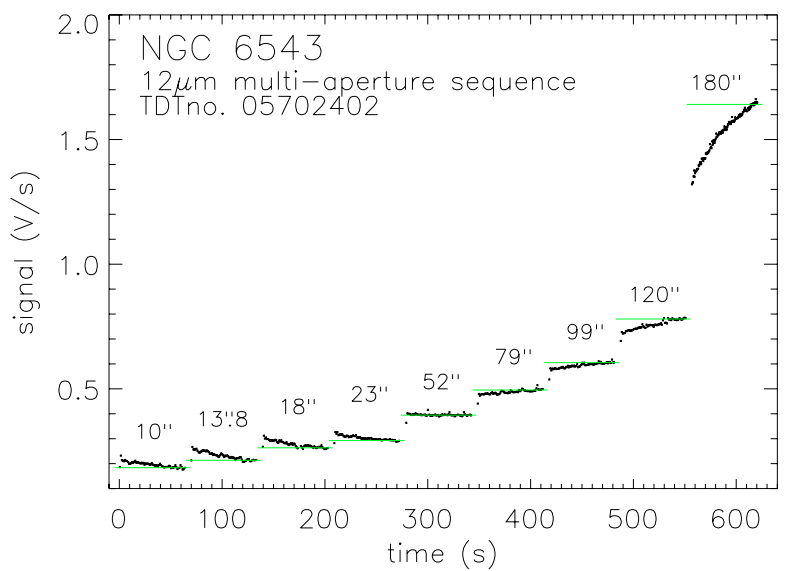

Fig. A.1. Signal behaviour along the sequence of 9 apertures between $10^{\prime \prime}$ and $120^{\prime \prime}$ for the $12 \mu \mathrm{m}$ measurement on NGC 6543. The green lines indicate the "average" signals computed by the processing software after drift recognition analysis. The signals in the 79", 120", and $180^{\prime \prime}$ apertures have been flagged as non-stabilised.

attempted to empirically model the transients by expressions with exponential functions of the form

$S(t)=S_{\infty}-\sum_{i=1}^{3} \Delta S_{i} \cdot \mathrm{e}^{\frac{t}{\tau_{i}}}$

similar to the form described by Acosta-Pulido et al. (2000) for the ISOPHOT FIR detectors. $S_{\infty}$ is the final signal level (at infinity), $\Delta S_{i}=S_{\infty} \pm S_{0}$ are positive or negative initial deviations and $\tau_{i}$ are characteristic time constants of the transients. Depending on the kind of transient $\Delta S_{2}$ and/or $\Delta S_{3}$ can be zero, if the transient is only downward or upward. Two and more time constants are only needed in the case of hook responses. There does not seem to be a unique time constant. Factors which determine the time constant are the illumination history and the flux jump at the beginning of the measurement. The extrapolation of the fit functions, displayed in Fig. A.2b, shows the corrected final signal values relative to the one from the standard products. Responsivity jumps in the signals due to glitches during the measurement have not been fitted by extra transient components; they would require also the introduction of time shifts $\Delta t$ in the numerator of the exponential function. Neither occasional superimposed oscillations have been attempted to be fitted.

For the $12 \mu \mathrm{m}$ multi-aperture sequence of NGC 6543 we found reasonable fits by applying time constants $\tau_{1}=50 \mathrm{~s}$ for the upwards trends and $\tau_{2}=40 \mathrm{~s}$ for the downward trends (see Fig. A.2 for examples). The average final signals determined from the last part of the transient curve by the standard processing are off with regard to these corrected values by $+6.4 \%$, $+7.6 \%,+5.2 \%,+3.2 \%,-2.0 \%,-2.1 \%,-3.5 \%$, and $-7.6 \%$ for the $10^{\prime \prime}, 13^{\prime \prime} 8,18^{\prime \prime}, 23^{\prime \prime}, 79^{\prime \prime}, 99^{\prime \prime}, 120^{\prime \prime}$, and $180^{\prime \prime}$ apertures, respectively. The $52^{\prime \prime}$ aperture signals need no correction.

In Fig. A.3 the transient corrected sequence is shown with the signals adjusted to the final level and the curvature taken out by subtracting the fit function. We fed the corrected signals back into PIA and completed the processing steps necessary for derivation of the normalised source profile.

Figure A.4 and Table A. 2 show a comparison of the source profiles obtained from automatic pipeline processing (OLP), i.e. the current ISO Data Archive product, from standard PIA processing and from PIA processing plus our transient correction. While the profiles look quite similar for apertures below $23^{\prime \prime}$, the transient corrected profile shows a significant difference: There 
U. Klaas et al.: ISOPHOT multi-aperture photometry. I., Online Material $p 6$
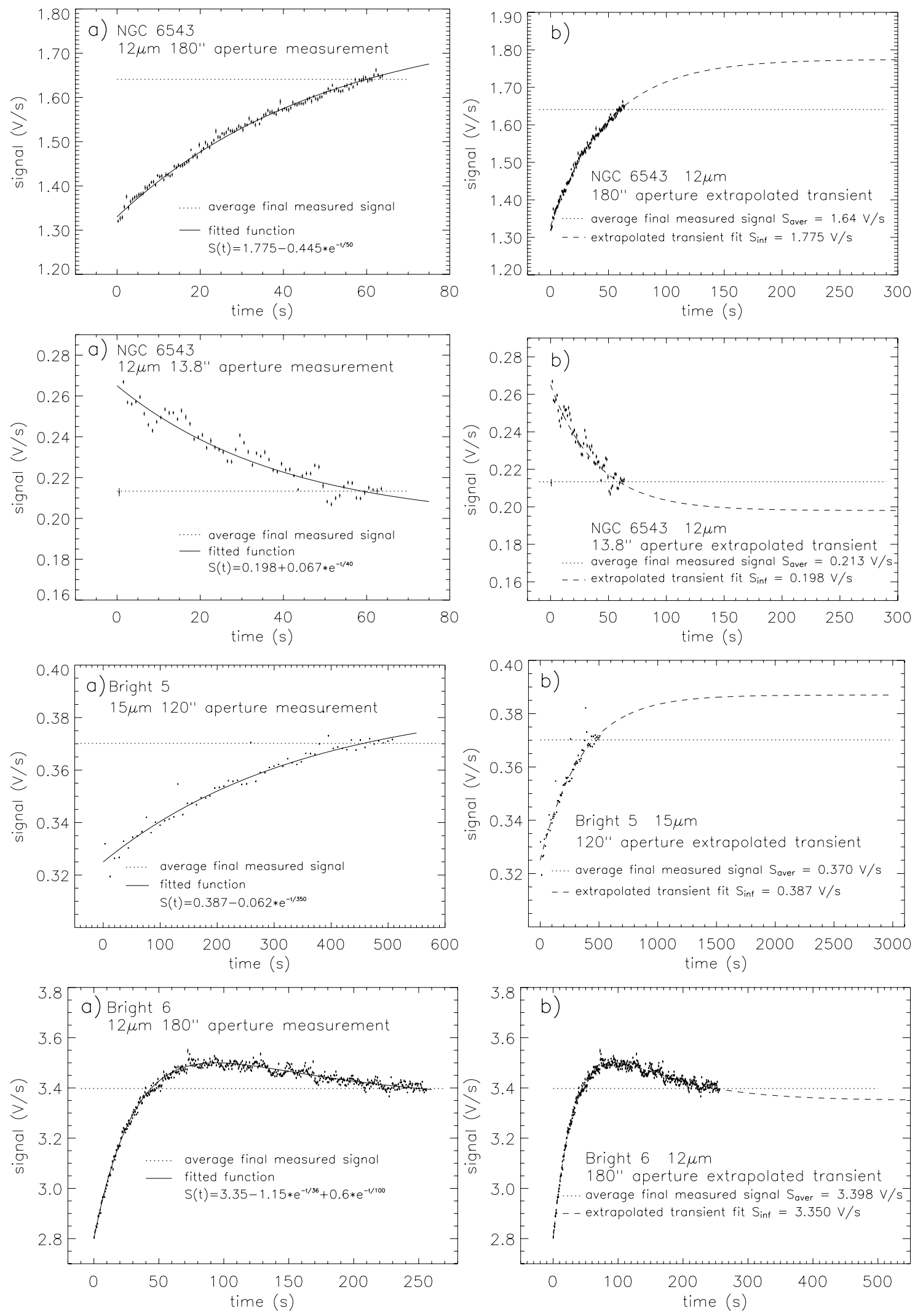

Fig. A.2. a) Zoomed view of measurements with transients. Error bars of the individual signals per ramp are indicated. The dotted line indicates the average of the final part of the signals as derived by the standard data processing. The solid line is a fit with a function of the form $S(t)=$ $S_{\infty}-\Delta S_{1} \cdot \mathrm{e}^{\frac{t}{\tau_{1}}}+\Delta S_{2} \cdot \mathrm{e}^{\frac{t}{\tau_{2}}} . \Delta S_{1}$ or $\Delta S_{2}$ may be zero, depending on whether the transient is upwards or downwards. Both $\Delta S$ are non-zero in case of a hook response (bottom panels). The parameters of the fit functions are indicated in the plot. b) Extrapolation of the fit functions (dashed line) to show the final signal level. 
U. Klaas et al.: ISOPHOT multi-aperture photometry. I., Online Material $p 7$

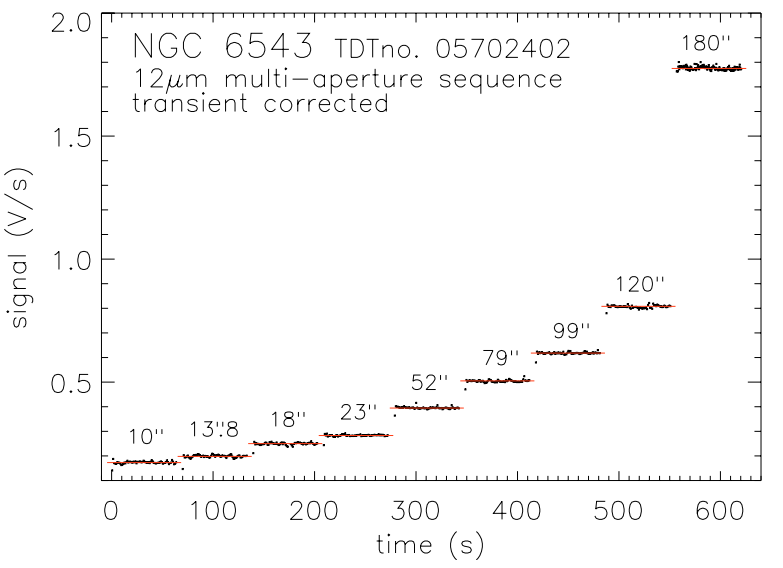

Fig. A.3. Transient corrected signals of the $12 \mu \mathrm{m}$ multi-aperture sequence on NGC 6543. The red lines indicate the final signal levels at infinity.

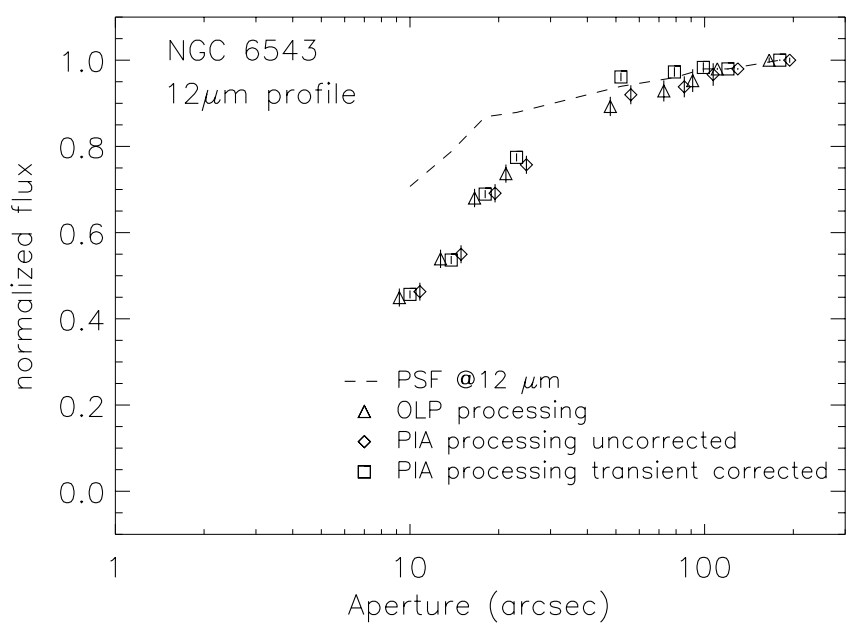

Fig. A.4. Comparison of source profiles resulting from the NGC 6543 $12 \mu \mathrm{m}$ multi-aperture observation by a) OLP Version 10 processing, identical with ISO Data Archive product, b) PIA Version 10 standard processing, c) PIA Version 10 processing with transient correction on signal per ramp (SRD) level. For better visibility the data points of a) and $\mathbf{b})$ are displaced to the left and right of the proper aperture position, respectively. Uncertainties have been calculated according to Eq. (A.10).

is no extended emission for aperture sizes greater equal 52", hence this is an artefact in the standard processed products. Uncertainties from OLP and uncorrected PIA processing are the same, while the transient corrected processing gives a gain of approximately a factor of 2.2 , because more signals of the measurement are used in the averaging process.

\section{A.1.5. Source profiles of point source standards}

Figure A.5 shows the resulting profiles for point sources which are also photometric standard stars. These are HR 617 ( $\alpha$ Ari: K2III), HR 1457 ( $\alpha$ Tau: K2III), HR 6705 ( $\gamma$ Dra: K5III), and HR7127 ( $\omega$ Pav: K2III). Details of the observations are given in Table A.6. In order to ensure the best quality reference profiles for point sources all measurements were processed interactively and transient correction as described in Sect. A.1.4 was performed, see Fig. A.12 for details. The corrections for the final signals were usually small, typically $1-2 \%$, however, straightening the time series of the signals led to a considerable reduction of the signal uncertainty.

By the definition of the procedure for constructing the source profiles (Sect. A.1.2) they should lie on the curve determined by the theoretical PSF fractions. For 3.6, 12, 20, 25, and $60 \mu \mathrm{m}$ there is good agreement for the larger apertures. For the smaller ones some deviations of the measured profiles towards smaller values than the PSF ones are observed, which would correspond to a slight broadening of the profiles. The exact explanation for this is currently not known, but multiple instrumental effects like slight telescope mis-pointings (partially accounted for in the error bars), asymmetries in the beam profiles, etc. may play a role. As pointed out in Sect. A.1.2, the empirical non-linear aperture correction does not play any role anymore, since the background contribution is practically not measurable.

The $7.3 \mu \mathrm{m}$ profile of HR 7127 shows a peculiar behaviour with a significant deviation from the theoretical profile, despite signal transient correction. However, this observation was the very last ISOPHOT observation, on revolution 875 after helium boil-off, when the cryostat was warming up and the detector was operated under non-standard and non-stable conditions. It is therefore not such a reliable PSF reference.

\section{A.1.6. Source profiles of the flat sky background}

For flat sky backgrounds the application of the background subtraction and normalisation procedure outlined in Sect. A.1.2 should ideally result in the profile dropping to zero for apertures with diameters smaller than $120^{\prime \prime}$ because of zero source signal, $S_{\text {aper }}^{\text {source }}=0$. Practically, due to noise and non-perfect aperture scaling corrections, the derivation of the "source" signal for each aperture leads to small residual values, $S_{\text {aper }}^{\text {soure }}= \pm \varepsilon$, and dividing small numbers by each other in the normalisation (Eq. A.6) can result in relatively large ratios and hence strong gradients between neighboring measurement points can result.

In order to ensure the best quality reference profiles for flat sky background sequences also all these measurements were processed interactively and transient correction as described in Sect. A.1.4 was performed. Figure A.6 shows examples of such sequences. Observations of this type are compiled in Table A.6 with the flag "flat sky". There are several sequences at 25 and $60 \mu \mathrm{m}$ which show the relatively good reproducibility of the profiles with the strong negative dip at $52^{\prime \prime}$ and $79^{\prime \prime}$ aperture size. The $15 \mu \mathrm{m}$ profile behaves similarly. The $20 \mu \mathrm{m}$ profile on the other hand looks much shallower and the $7.3 \mu \mathrm{m}$ profiles and $12 \mu \mathrm{m}$ profile show partially values above 1.0 for the $79^{\prime \prime}$ and $99^{\prime \prime}$ apertures. This is due to the fact that the residuals remain all positive and are slightly larger than for the $180^{\prime \prime}$ aperture.

One crucial step in the analysis of multi-aperture profiles is therefore to determine the source-to-background contrast for the $180^{\prime \prime}$ aperture, $\frac{S_{180}^{\text {source }}}{S_{180}^{\text {bg }}}$. When the source flux makes up only a few per cent of the background flux in the 180" aperture, care is needed and it is mandatory to do a comparison with the flat sky background sequences before interpreting the source profile. In particular, at $20 \mu \mathrm{m}$, the flat sky background profile mimics a broad Gaussian distribution.

\section{A.2. Modelling and quantitative interpretation of multi-aperture profiles}

In order to interpret the measured profiles in some quantitative way, the profiles of theoretical intensity distributions are 
Table A.2. Comparison of normalised source profiles and their uncertainties derived with a) OLP Version 10 processing, identical with ISO Data Archive product, b) PIA Version 10 standard processing, c) PIA Version 10 processing with transient correction on signal per ramp (SRD) level. For the OLP product two uncertainties are given: 1) the standard one provided in the ISO Data Archive product and 2) a revised one calculated according to Eq. (A.10).

\begin{tabular}{rccccccc}
\hline \hline $\begin{array}{r}\text { Aperture } \\
\left({ }^{\prime \prime}\right)\end{array}$ & source & OLP 10 & standard & revised & \multicolumn{2}{c}{ PIA 10 standard } & \multicolumn{2}{c}{ PIA 10+ transient correction } \\
& profile & uncertainty & uncertainty & revised & source & revised \\
profile & uncertainty & profile & uncertainty \\
\hline 10 & 0.449 & 0.005 & 0.021 & 0.463 & 0.021 & 0.457 & 0.009 \\
13.8 & 0.539 & 0.006 & 0.021 & 0.550 & 0.021 & 0.536 & 0.009 \\
18 & 0.680 & 0.007 & 0.021 & 0.691 & 0.021 & 0.689 & 0.009 \\
23 & 0.737 & 0.006 & 0.021 & 0.757 & 0.021 & 0.775 & 0.009 \\
52 & 0.893 & 0.008 & 0.022 & 0.920 & 0.022 & 0.961 & 0.010 \\
79 & 0.929 & 0.008 & 0.024 & 0.938 & 0.024 & 0.973 & 0.011 \\
99 & 0.953 & 0.008 & 0.026 & 0.967 & 0.026 & 0.983 & 0.012 \\
120 & 0.980 & 0.009 & 0.002 & 0.980 & 0.002 & 0.980 & 0.002 \\
180 & 1.000 & 0.012 & 0.006 & 1.000 & 0.006 & 1.000 & 0.002 \\
\hline
\end{tabular}

determined for comparison. These are superpositions of the two simple components

1) Gaussians (with various $F W H M$ );

2) Point Spread Functions (PSF),

with various intensity ratios and with various offsets with regard to the origin. For these calculations relative intensities are used.

\section{A.2.1. Basic method}

For a given intensity distribution, the local intensity is calculated on a $181 \times 181$ grid with a spatial resolution of $1^{\prime \prime}$ (for the shortest wavelengths $<5 \mu \mathrm{m}$ a $361 \times 361$ grid with a spatial resolution of 0.5 has been chosen),

$I\left(r_{x_{i}, y_{j}}=\sqrt{x_{i}^{2}+y_{j}^{2}}\right)$

$-90 \leq x_{i} \leq+90,-90 \leq y_{j} \leq+90, \Delta x_{i}, \Delta y_{j}=1$.

For each aperture, the intensity is summed up within the radius of the aperture

$I_{\text {aper }}=\sum_{i, j}^{r_{x_{i}, y_{j}} \leq r_{\text {aper }}} I\left(r_{x_{i}, y_{j}}\right)$

and then the same procedure as for the measured signals is used, except that the empirical aperture correction factors $\left(a_{\text {aper }}^{\text {corr }}\right)$ are not applied, since the theoretical intensities have been calculated for an ideal flat aperture response $(=1$ inside aperture, $=0$ outside). Using Eqs. (A.5), (A.4), and (A.6) the normalised model flux profiles are derived.

\section{A.2.2. Source profiles of Gaussians}

One of the simplest extended source intensity distributions is a Gaussian which would correspond to a shell-like source structure. The intensity distribution is given by

$I(r)=\mathrm{e}^{-0.5 \cdot\left(\frac{r}{\sigma_{1,2}}\right)^{2}}$

with $\sigma_{1,2}=\frac{x_{1,2}}{2 \cdot \sqrt{2 \cdot \log (2)}}, \mathrm{x}_{1,2}=F W H M$. For a given wavelength the $F W H M$ is never chosen smaller than the $F W H M$ of the PSF which is approximately given by

$x_{1,2}^{P S F} \quad\left[{ }^{\prime \prime}\right]=0.336 \cdot \lambda_{\mathrm{c}}[\mu \mathrm{m}]$.
Figure A.7 shows the profiles for a suite of Gaussians with FWHM between $5^{\prime \prime}$ and $100^{\prime \prime}$. Two features of the Gaussian profiles are obvious:

1) the profiles with a $F W H M$ equal to the $F W H M$ of the PSF $\left(\approx 5^{\prime \prime}\right.$ at $\left.15 \mu \mathrm{m}\right)$ rise faster close to a value of 1 and flatten out afterwards;

2) the curves with large $F W H M$ lie quite close to each other and an increase of the FWHM beyond $100^{\prime \prime}$ does not push the curves much farther towards smaller normalised flux values for the corresponding aperture.

\section{A.2.3. Source profiles of double point sources}

For simplicity and reasonable computation times the PSF of the ISO telescope has been modelled as the intensity distribution of a folded two-mirror system with central obscuration $\varepsilon$ (cf. Schroeder 1987).

With

$v=\frac{\pi}{\lambda \cdot F_{\text {rat }}} \cdot r$

$F_{\text {rat }}=15$ being the telescope aperture ratio, the PSF can be described as

$\operatorname{PSF}(v)=\frac{1}{\left(1-\varepsilon^{2}\right)^{2}} \cdot\left(\frac{2 J_{1}(v)}{v}-\varepsilon^{2} \cdot \frac{2 J_{1}(\varepsilon v)}{\varepsilon v}\right)^{2}$.

The intensity distribution of a double point source is then described by

$I(r)=\operatorname{PSF}\left(v_{1}\right)+\alpha \cdot \operatorname{PSF}\left(v_{2}\right)$

with $r_{1}-r_{2}=d_{1,2}$ being the offset between the two point sources and $\alpha$ their relative intensity ratio.

Figure A. 8 shows the profiles for a suite of double PSFs with distances between $10^{\prime \prime}$ and $80^{\prime \prime}$ and one being centered in the origin. Two different families of curves can be recognized. Up to a distance of $50^{\prime \prime}$ they steadily rise up to the fixed value for the $120^{\prime \prime}$ aperture. From $d_{1,2}=60^{\prime \prime}$ onward the shape of the profile changes significantly, for distances of $70^{\prime \prime}$ and $80^{\prime \prime}$ it is completely above that of the single PSF. The reason is that a significant fraction of the intensity of the second offset source is 
U. Klaas et al.: ISOPHOT multi-aperture photometry. I., Online Material $p 9$
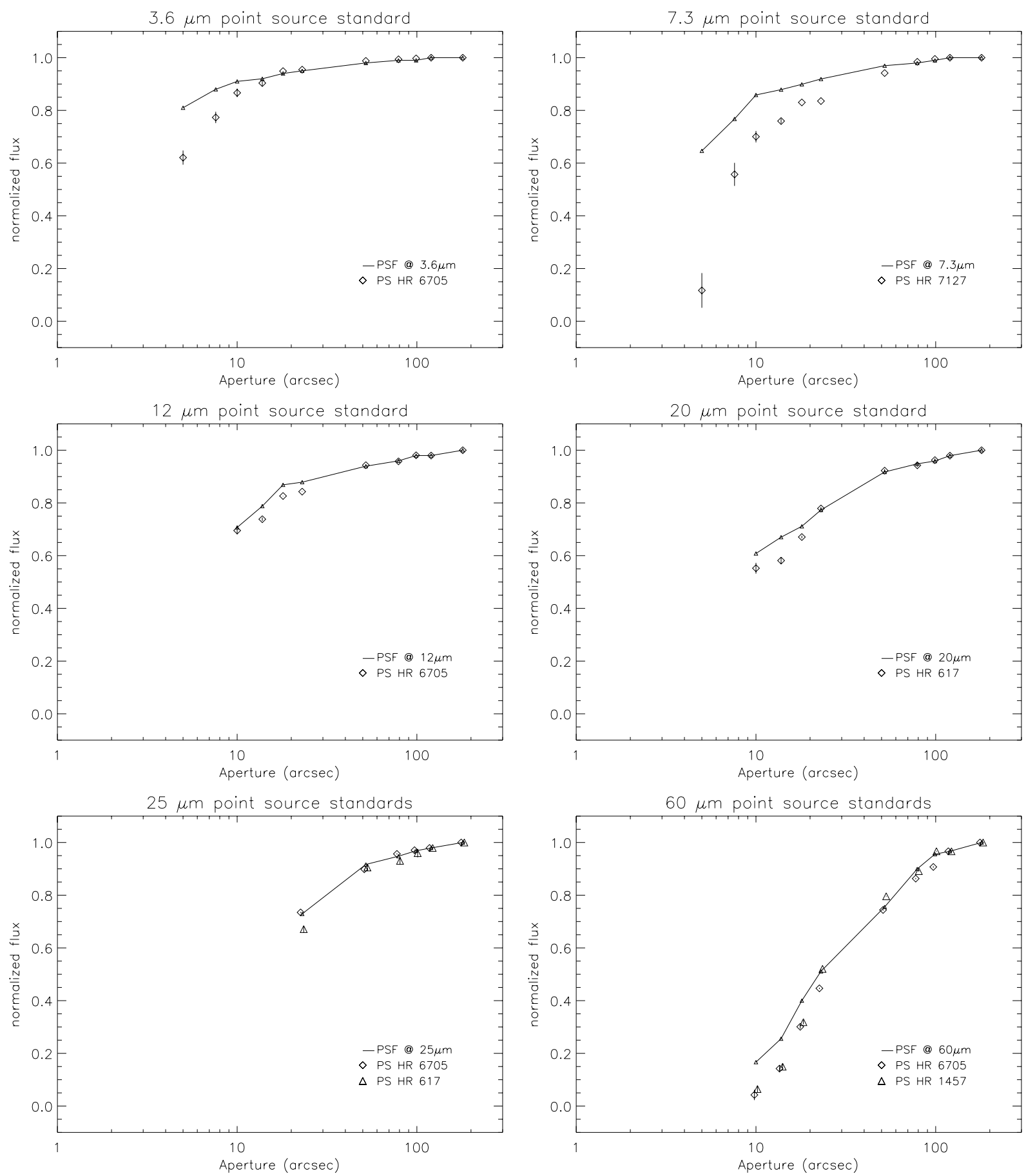

Fig. A.5. Multi-aperture profiles of point source standards. Note that, in the case where two stars are plotted on the same graph, the data points have been slightly displaced with regard to the aperture value for better visibility. The course of the theoretical Point Spread Function (PSF) is indicated by the solid line, measured data points of the point source (PS) reference profiles are labelled by either a " $\diamond$ " or a " $\triangle$ ". Error bars represent the uncertainties in the signal processing as outlined in Sect. A.1.3. For the smallest apertures $\left(\leq 18^{\prime \prime}\right)$ uncertainties due to telescope pointing jitter have been added.

located in the annulus between the $120^{\prime \prime}$ and the $180^{\prime \prime}$ aperture rims and is therefore subtracted as background. This leads to the result that the source appears to be "narrower" than a point source. Therefore, these profiles represent a definite signature of this particular source configuration.

\section{A.2.4. Ring-like structures}

Since the integration of the intensity is done inside circular annuli, it cannot be distinguished between spot-like sources, like point sources, and intensities distributed over rings. An equivalent intensity distribution to the one described in Eq. (A.16) is therefore

$I(r)=\operatorname{PSF}\left(v_{0}\right)+\sum_{i=1}^{n_{\text {ring }}} \beta \cdot \operatorname{PSF}\left(v_{i}\right)$,

with $\left|r_{i}\right|=r_{\text {ring. }}$. The factor $\beta$ is related to the factor $\alpha$ in Eq. (A.16) via $\beta=\frac{\alpha}{n_{\text {ring }}}$, with $n_{\text {ring }}$ being the integer part of $\pi \cdot \frac{r_{\text {ring }}}{1^{\prime \prime}}$, using a step size of $2^{\prime \prime}$ in the spacing of the ring PSFs. 
U. Klaas et al.: ISOPHOT multi-aperture photometry. I., Online Material p 10
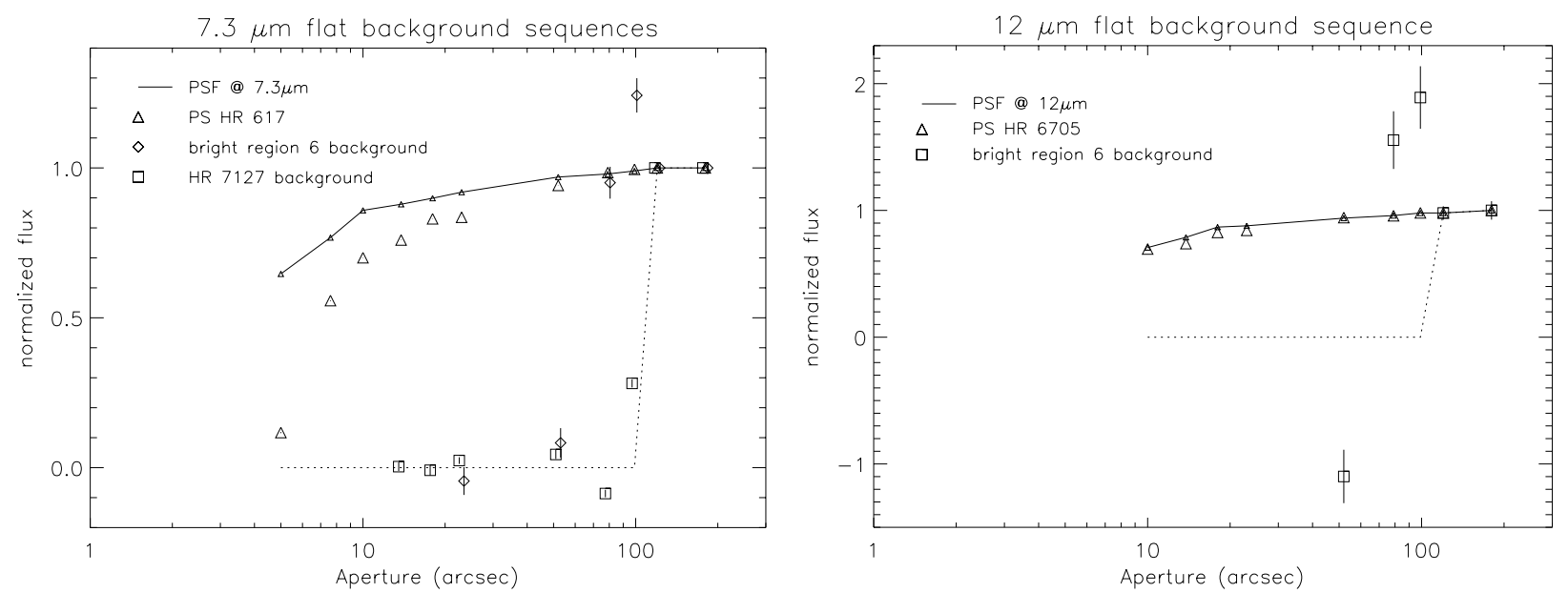

$15 \mu \mathrm{m}$ flat background sequence
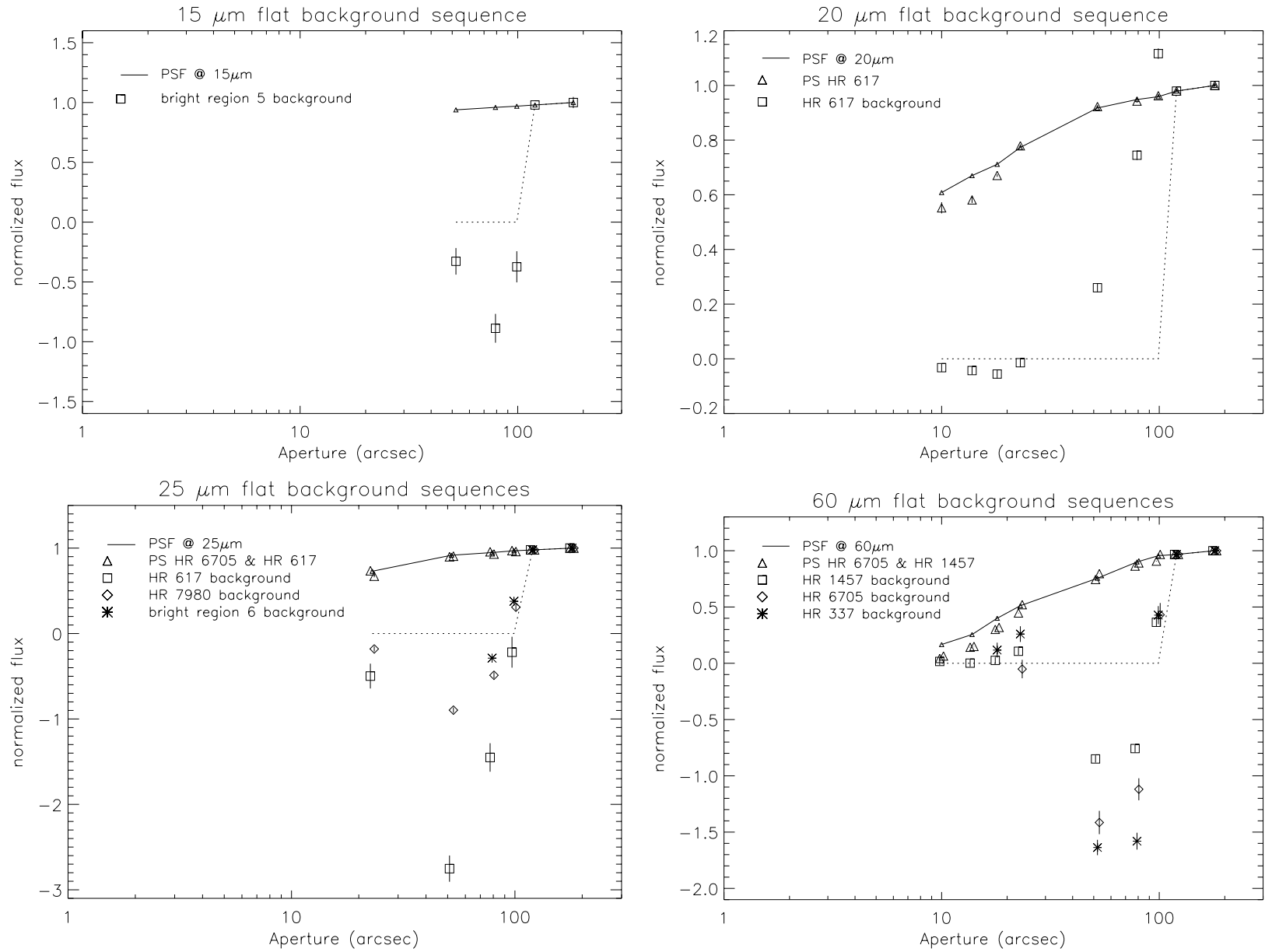

Fig. A.6. Multi-aperture profiles of flat sky backgrounds. The dotted line outlines the ideal behaviour for such a sequence. Note that, in the case where two or more backgrounds are plotted on the same graph, the data points have been slightly displaced with regard to the aperture value for better visibility. The course of the theoretical Point Spread Function (PSF) is indicated by the solid line, measured data points of the point source (PS) reference profiles are always indicated by a " $\triangle$ ". The data points of the background profiles are labelled by either a " $\square$ ", a " $\diamond$ ", or a “ $* "$. Error bars represent the uncertainties in the signal processing as outlined in Sect. A.1.3.

For an elliptical ring the number of ring sources is $n_{\mathrm{ell}}$ ring $=$ $\frac{\pi}{2} \cdot\left(1.5 \cdot \frac{a}{1^{\prime \prime}} \cdot\left(\frac{b}{a}-1\right)-\frac{a}{1^{\prime \prime}} \cdot \sqrt{\frac{b}{a}}\right)$ with a and $b$ being the major and minor axis length, respectively. Due to the superposition of the point sources making up the ring the resulting peak intensity of the ring is related to the peak intensity of the single point source by

$I_{0}^{\mathrm{PSFring}} \approx \frac{1.834 \cdot \lambda[\mu \mathrm{m}]}{10[\mu \mathrm{m}] \cdot n_{\text {ring }}} \cdot I_{0}^{\text {singlePSF }}$ with the radius of the ring $r_{\text {ring }}$ being equivalent to the offcentre distance $d_{\text {off }}$ of the individual point source. The factor 1.834 is approximately the integral of PSFs along one axis, $\sum_{i=-n}^{+n} \operatorname{PSF}\left(x_{i}\right), n \geq 40, \Delta x_{i}=2^{\prime \prime}$ for $\lambda=10 \mu \mathrm{m}$. The upper panel of Fig. A.9 demonstrates this equivalence of the normalized flux for an intensity uniformly distributed in a ring to the off-centre point source intensity distribution as shown in the upper panel of Fig. A.8. 


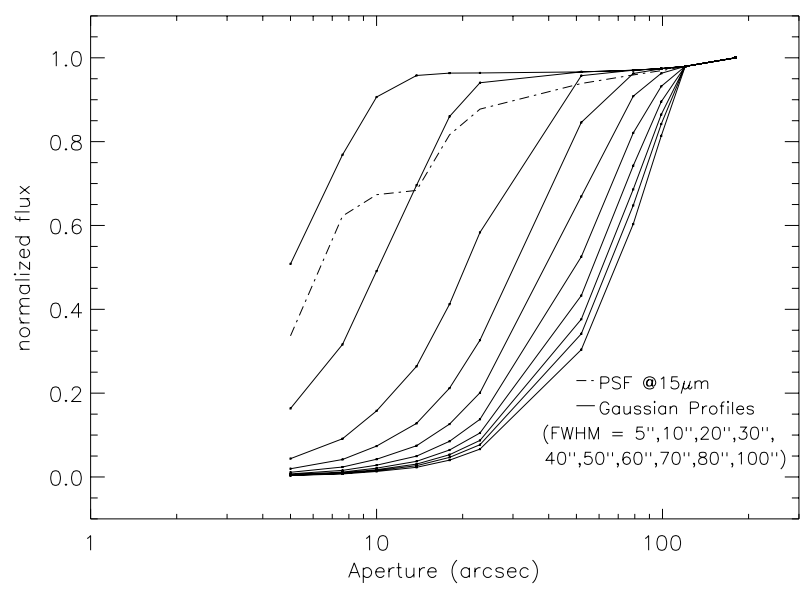

Fig. A.7. Suite of Gaussian profiles with a FWHM between $5^{\prime \prime}$ (most left solid curve) and 100" (most right solid curve) centered at the origin of the apertures. For comparison the profile of a point source at $15 \mu \mathrm{m}$ is drawn (dashed-dotted line). Please note that the normalised flux is only calculated for the actual ISOPHOT aperture diameters, and these points are connected by straight lines.

In case of a Gaussian ring the relation is

$$
I_{0}^{\text {Gauss ring }} \approx \frac{5.322 \cdot F W H M\left[^{\prime \prime}\right]}{10\left[{ }^{\prime \prime}\right] \cdot n_{\text {ring }}} \cdot I_{0}^{\text {single Gaussian }} \text {. }
$$

Here, the factor 5.322 is the integral of Gaussian functions along one axis, $\sum_{i=-n}^{+n} \mathrm{e}^{-0.5 \cdot\left(\frac{x_{i}}{\sigma_{1,2}}\right)^{2}}, n \geq 40, \Delta x_{i}=2^{\prime \prime}$ for a $F W H M=10^{\prime \prime}$.

\section{A.2.5. More complex 2-D structures}

In particular for shorter wavelengths, where the spatial resolution improves, a two component intensity distribution does often not reflect all the details seen in the multi-aperture profile. Figure A.10 shows an example how several different intensity distributions can yield the same radial flux profile within the measurement uncertainties.

The first case is a triple point source, a central one and two off-centre point sources. The three sources need not to be aligned as in the example, they can be located at any angle. The second case is a central point source plus two unresolved rings (made up of PSFs). The third case is a central point source plus an unresolved elliptical ring centered on the point source and variable intensity along the ring with the highest intensities in the two points on the minor axis. The fourth case is a central point source plus an unresolved eccentric elliptical ring (shifted in $x$-direction) and variable intensity along the ring with the highest intensity in the point closest to the central point source.

Despite the differences in the 2D appearance of these four intensity distributions there are some commonalities. In Table A.3 we compare several of the crucial model parameters. It can be seen that both the radial distances of important source structures and the total intensities are quite similar. Therefore, it is important to note that the solution given by the "linear" source model (all components on the $x$-axis) provides a first good guess on the distribution and relative intensity of the extended emission.

\section{A.2.6. Ranges of model parameters}

The model parameters, like relative intensity ratios, relative distances or FWHM are adjusted such that the profile of the adopted
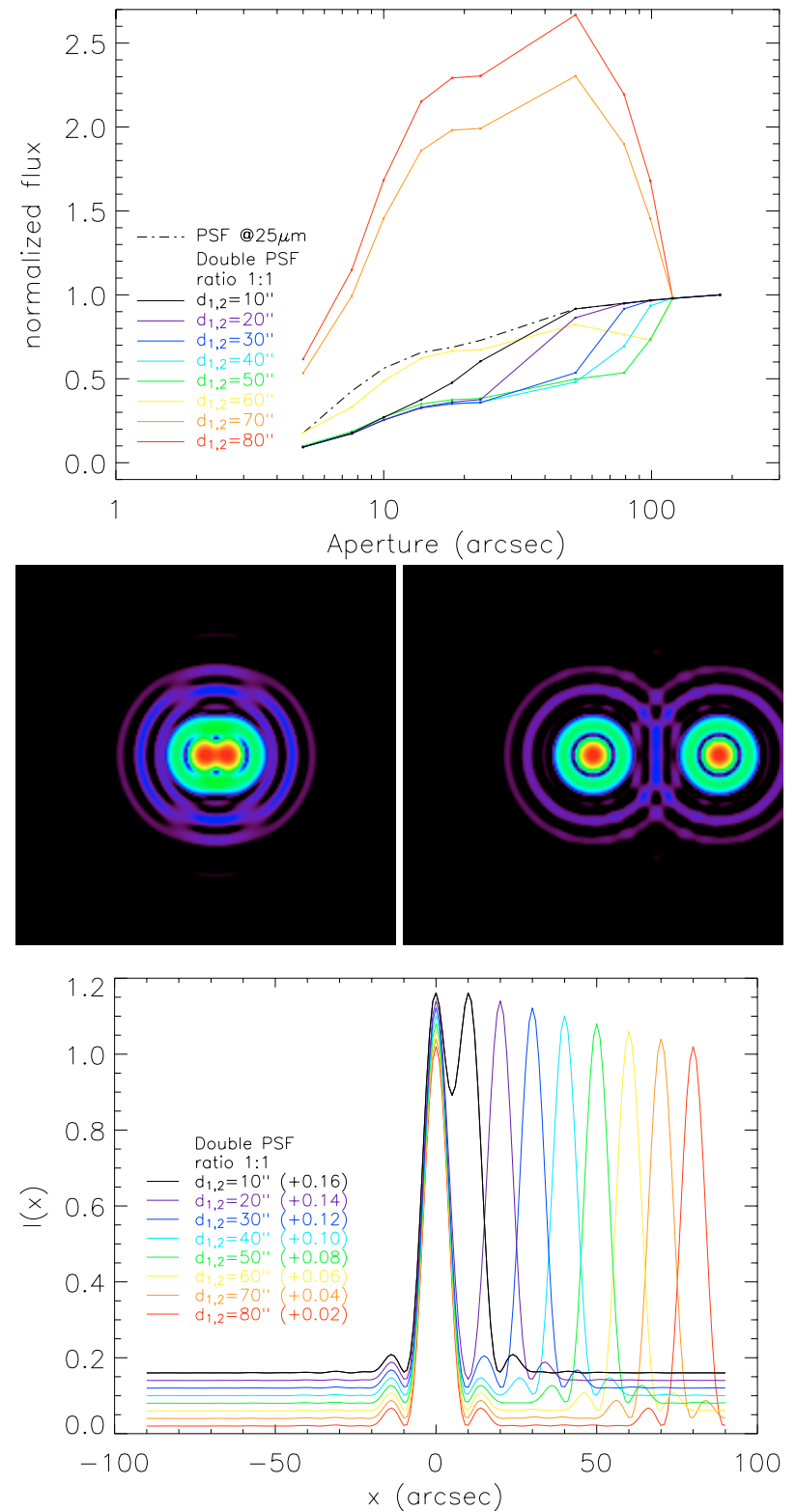

Fig. A.8. Upper panel: multi-aperture profiles for two equally bright point sources at various distances $d_{1,2}=10-80^{\prime \prime}$, in steps of $10^{\prime \prime}$. For comparison the profile of a single point source (PSF) at $25 \mu \mathrm{m}$ is drawn. The normalised flux is only calculated for the actual ISOPHOT aperture diameters, and these points are connected by straight lines. Middle two panels: false colour images of the intensity distribution in the $181 \times$ 181 grid for the two cases with $d_{1,2}=10^{\prime \prime}$ and $d_{1,2}=60^{\prime \prime}$, respectively. Lower panel: cut through the intensity distributions along the $x$-axis. For better visibility the profiles are offset by steps of +0.02 , increasing from the largest distance to the smaller ones.

model fits the measured data points as close as possible. Each individual parameter is then varied so that the model profile is still within the error bars of the measurement points. The parameters of the best fit and the parameter ranges corresponding to the measurement uncertainties are then taken as the best parameter values and their uncertainties for the specific model. This does not rule out considerably different intensity distribution models which fit the radial profile equally well as shown in the previous section. 

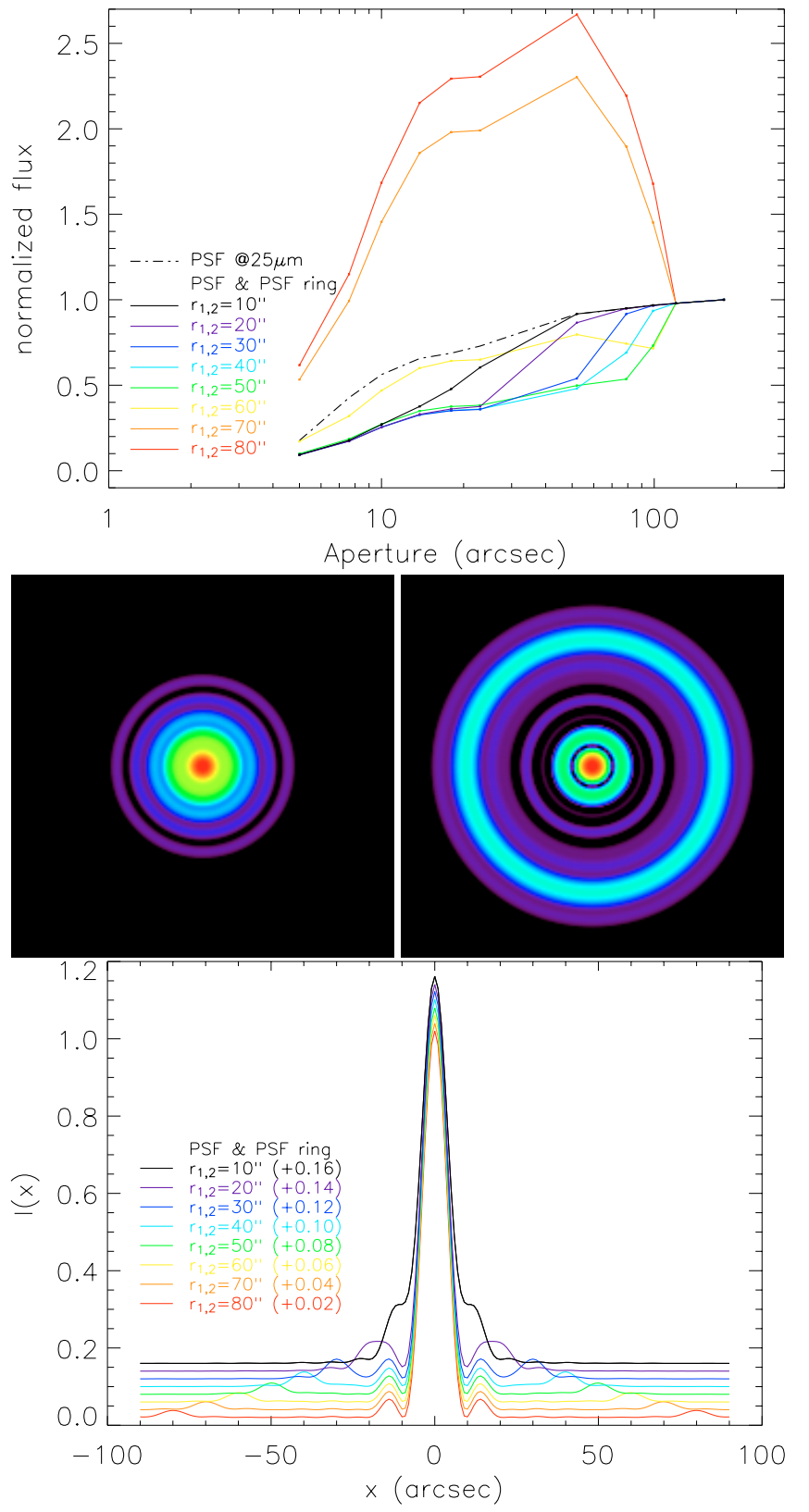

Fig. A.9. Upper panel: multi-aperture profiles for a point source surrounded by a "ring of point sources" at various radii $r_{1,2}=10-80^{\prime}$, in steps of $10^{\prime \prime}$. For comparison the profile of a point source (PSF) at $25 \mu \mathrm{m}$ is drawn. The normalised flux is only calculated for the actual ISOPHOT aperture diameters, and these points are connected by straight lines. Middle two panels: false colour images of the intensity distribution in the $181 \times 181$ grid for the two cases with $r_{1,2}=10^{\prime \prime}$ and $r_{1,2}=60^{\prime \prime}$, respectively. Lower panel: cut through the intensity distributions along the $x$-axis. For better visibility the profiles are offset by steps of +0.02 , increasing from the largest radius to the smaller ones.

\section{A.3. Photometry from multi-aperture sequences}

\section{A.3.1. Assignment of absolute flux level}

One issue listed in Sect. A.1.1 was that the internal Fine Calibration Source could not be calibrated against celestial standards in combination with the $180^{\prime \prime}$ aperture for the P1 and P2 detectors. This, however, turned out to be no handicap, since both detectors were relatively stable during the mission and the orbit dependent default responsivities can be applied, see the
ISOPHOT Handbook (Laureijs et al. 2003) for more details. For the P3 detector, the $180^{\prime \prime}$ aperture was the default calibration aperture and therefore the actual responsivity values can be derived from the FCS measurement.

$R\left[\mathrm{~A} \mathrm{~W}^{-1}\right]=\left\{\begin{aligned} R\left(\phi_{\text {orbit }}\right) & \text { for } P 1, P 2 \\ R(t) & \text { for } P 3\end{aligned}\right.$

For the derivation of the fluxes the source signals $S_{180}^{\text {source (in units }}$ of $\mathrm{V} \mathrm{s}^{-1}$ ) as listed in the SPD product (Laureijs et al. 2003) headers under keywords "SRCSIG" are used. They are converted to a detector inband power, $P_{180}$

$P_{180}=\frac{S_{180}^{\text {source }} \cdot C_{\text {int }}}{R} \quad[\mathrm{~W}]$

with $C_{\text {int }}=9.0 \times 10^{-14} \mathrm{~A} \mathrm{~s} \mathrm{~V}^{-1}$ being the integration capacity of the cold read-out electronics. Note that in the case of interactive signal processing and making use of the SCP data (Signal per Chopper Plateau; for the multi-aperture observations which are in staring mode this is the average signal of the entire measurement in one aperture) the relative photometric filter-to-filter correction (cf. chapter 7.10.3 in Laureijs et al. 2003) has to be applied before in order to get consistent photometric results:

$$
S_{\text {phot }}^{\mathrm{IA}}=\frac{S_{\mathrm{SCP}}^{\mathrm{IA}}}{\chi_{\text {det }}(\text { filter })} .
$$

For convenience we have compiled these corrections factors from CALG file PPFTOF in Table A.4.

The monochromatic flux densities $F_{v}$ (for SED $F_{v} \cdot v=$ const.) are then derived by applying the band conversion factors, $C_{1}\left(\lambda_{\mathrm{c}}\right)$ (in units of $\mathrm{m}^{2} \mathrm{~Hz}$ ), as listed in the CALG file PFLUXCONV (Laureijs et al. 2003).

$$
F_{v, 180}=\frac{P_{180}}{C_{1}\left(\lambda_{\mathrm{c}}\right) \cdot 10^{-26} \cdot f_{180}^{\mathrm{psf}}} \quad[\mathrm{Jy}] .
$$

There are two sources of uncertainty in the flux determination. These are

1) the uncertainty in the source signal. This is already an error propagation of the signal uncertainties obtained for the individual aperture measurements;

2) the uncertainty of the signal in the responsivity measurement.

Since both are of statistical nature, Gaussian error addition applies

$$
\frac{\Delta F_{v}}{F_{v}}=\sqrt{\left(\frac{\Delta S_{180}^{\text {source }}}{S_{180}^{\text {source }}}\right)^{2}+\left(\frac{\Delta R}{R}\right)^{2}} .
$$

For the standard stars presented in Sect. A.1.5 we compare the derived flux densities, appropriately colour corrected (see Laureijs et al. 2003, Appendix C), with their photometric model fluxes in Table A.5. It can be seen that quite good consistency with the model fluxes is achieved, the agreement with the model deteriorates when the source-to-background contrast, $\frac{S_{180}^{\text {source }}}{S_{180}^{\mathrm{bg}}}$ decreases. 
U. Klaas et al.: ISOPHOT multi-aperture photometry. I., Online Material p 13
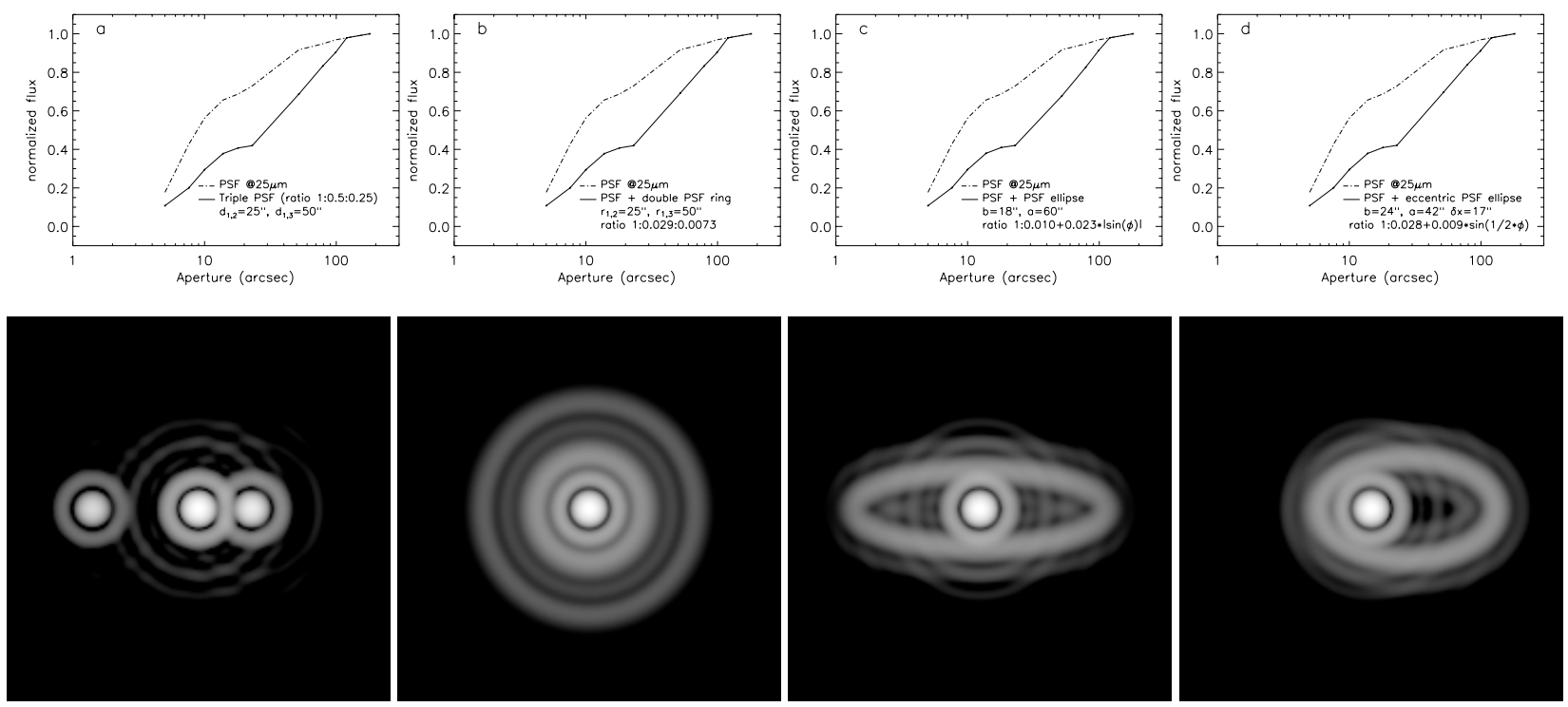

Fig. A.10. 2D intensity distributions at $25 \mu \mathrm{m}$ (lower panel) which result in a similar radial profile (upper panel): a) triple PSF (note: the sources need not to be linearly aligned), the two off-centre sources are at $d_{1}=25^{\prime \prime}$ and $d_{2}=50^{\prime \prime}$, respectively, the relative peak intensity ratio is 1:0.5:0.25 from the centre outwards; b) central PSF plus double PSF ring with radii $r_{1}=25^{\prime \prime}$ and $r_{2}=50^{\prime \prime}$, respectively, the resulting relative peak intensity ratio is 1:0.029:0.0073; c) central PSF plus elliptical PSF ring with semi-major axis $a=60^{\prime \prime}$ and semi-minor axis $b=18^{\prime \prime}\left(\frac{b}{a}=0.3\right)$, the intensity along the ring, made up of 132 individual PSFs, varies with $0.010+0.023 \cdot|\sin (\phi)|$ with the highest intensity along the minor axis; d) central PSF plus eccentric elliptical PSF ring with semi-major axis $a=42^{\prime \prime}$ and semi-minor axis $b=24^{\prime \prime}\left(\frac{b}{a}=0.57\right)$, the shift in direction of the $x$-axis is $17^{\prime \prime}$, the intensity along the ring, made up of 105 individual PSFs, varies with $0.028+0.009 \cdot \sin \left(\frac{1}{2} \phi\right)$ with the highest intensity at the closest point to the central point source.

Table A.3. Comparison of the model parameters of the intensity distributions of Fig. A.10.

\begin{tabular}{ccc}
\hline \hline $\begin{array}{c}\text { Closest } \\
\text { off-centre } \\
\text { (") }\end{array}$ & $\begin{array}{c}\text { Farthest } \\
\left({ }^{\prime \prime}\right)\end{array}$ & $\begin{array}{c}\text { Total integrated relative } \\
\text { off-centre intensity }\end{array}$ \\
\hline$d_{1}=25$ & $d_{2}=50$ & 0.75 \\
$r_{1}=25$ & $r_{2}=50$ & 0.75 \\
$b=18$ & $a=60$ & 0.78 \\
$a_{1} \approx b \leq 25$ & $a_{2}=59$ & 0.78 \\
\hline
\end{tabular}

Table A.4. Relative photometric ISOPHOT-P filter-to-filter correction factors from CALG file PPFTOF to be applied to interactively processed signals according to Eq. (A.21).

\begin{tabular}{lrc}
\hline \hline Detector & $\lambda_{\mathrm{c}}^{\text {filter }}$ & $\chi_{\text {det }}($ filter $)$ \\
\hline P1 & 3.3 & 0.916 \\
P1 & 3.6 & 0.962 \\
P1 & 4.8 & 1.000 \\
P1 & 7.3 & 0.839 \\
P1 & 7.7 & 0.849 \\
P1 & 10.0 & 0.659 \\
P1 & 11.3 & 0.673 \\
P1 & 12.0 & 0.293 \\
P1 & 12.8 & 0.354 \\
P1 & 15.0 & 0.289 \\
P2 & 20.0 & 0.615 \\
P2 & 25.0 & 1.000 \\
P3 & 60.0 & 1.000 \\
P3 & 100.0 & 0.995 \\
\hline
\end{tabular}

\section{A.3.2. Reconstruction of the total source flux using the model in case of sources extending into the background annulus}

As pointed out in Sect. A.1.2 and shown by the examples in Figs. A. 8 and A.9 part of the source may be located inside the
Table A.5. Comparison between the colour corrected monochromatic flux densities derived for point source standards from the $180^{\prime \prime}$ aperture measurement of the multi-aperture sequences and model fluxes provided for the general photometric calibration of ISO (Cohen 2003; and Hammersley \& Jourdain de Muizon 2003, and references therein, see also http://www.iso.vilspa. esa.es/users/expl_lib/ISO/wwwcal/). Quoted flux uncertainties calculated according to Eq. (A.23) take only statistical uncertainties of the signal determination into account and correspond to $1 \sigma . \Delta$ gives the consistency with regard to the model flux $\left|\frac{F_{180}^{\mathrm{cc}}-F_{\text {model }}}{F_{\text {model }}}\right| . \frac{S_{180}^{\text {source }}}{S_{180}^{\mathrm{bg}}}$ is the source-to-background contrast.

\begin{tabular}{lccccc}
\hline \hline Name & $\begin{array}{c}\lambda_{\mathrm{c}} \\
(\mu \mathrm{m})\end{array}$ & $\begin{array}{c}F_{180}^{\mathrm{cc}} \\
(\mathrm{Jy})\end{array}$ & $\begin{array}{c}F_{\text {model }} \\
(\mathrm{Jy})\end{array}$ & $\begin{array}{c}\Delta \\
(\%)\end{array}$ & $\begin{array}{c}\frac{S_{180}^{\text {souce }}}{S_{180}^{\text {bg }}} \\
(\%)\end{array}$ \\
\hline HR 6705 & 3.6 & $991 \pm 9$ & 1031 & 4 & $>1000$ \\
HR 6705 & 12 & $103 \pm 3$ & 109 & 6 & 400 \\
HR 617 & 20 & $17.3 \pm 0.3$ & 20.5 & 16 & 36 \\
HR 617 & 25 & $11.3 \pm 0.3$ & 13.2 & 14 & 26 \\
HR 6705 & 25 & $24.0 \pm 0.3$ & 25.2 & 5 & 122 \\
HR 7980 & 25 & $5.6 \pm 0.2$ & 6.5 & 14 & 14 \\
HR 1457 & 60 & $18.9 \pm 0.3$ & 19.1 & 1 & 136 \\
HR 6705 & 60 & $4.9 \pm 0.1$ & 4.5 & 9 & 170 \\
\hline
\end{tabular}

annulus between the $120^{\prime \prime}$ and $180^{\prime \prime}$ aperture borders, so that part of the source signal will be subtracted as background. With the intensity ratio $\frac{I_{120}^{\text {model }}}{I_{180}^{\text {model }}}$ from the model it is possible to reconstruct a total source flux from the uncorrected signals. For this purpose the model is recalculated on a larger grid extending out to $\pm 180^{\prime \prime}$ and the intensity within a $360^{\prime \prime}$ aperture is set to $1, I_{360}^{\text {model }}=1.0$.

The uncorrected source and background signals are given in the SPD file headers and we will now establish a relation between the corrected source signal in the 180" aperture 
U. Klaas et al.: ISOPHOT multi-aperture photometry. I., Online Material p 14
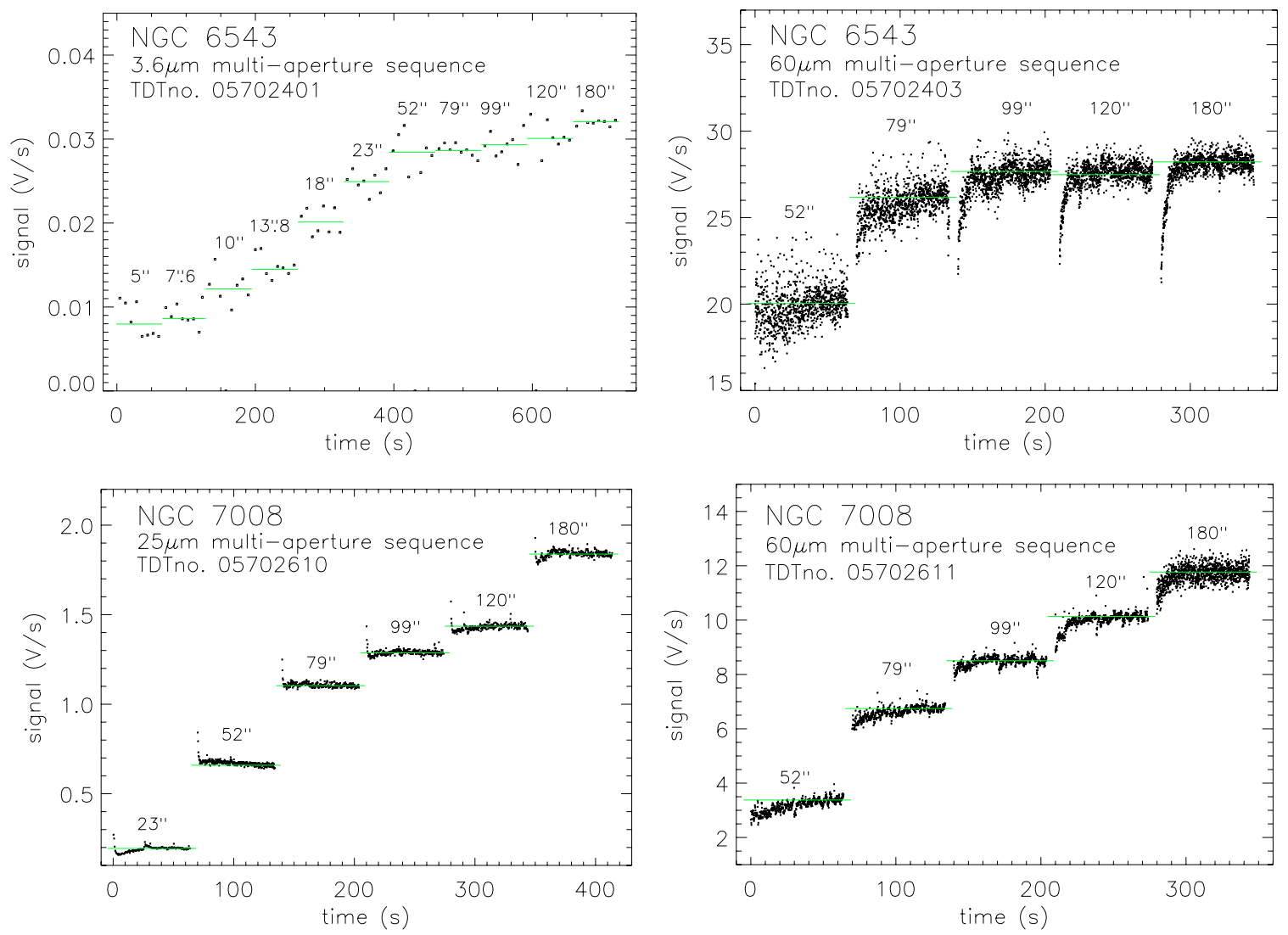

Fig. A.11. Signal time series of multi-aperture sequences on the PNe NGC 6543 and NGC 7008 discussed in the main part of the paper.

and the uncorrected source and background signals of the $120^{\prime \prime}$ and $180^{\prime \prime}$ apertures.

The total signal in any aperture remains constant, only the relative contributions of source and background signals will change. Hence, Eq. (A.1) is fulfilled both for the uncorrected signals, $S_{\mathrm{u}}$ and the corrected signals, $S_{\mathrm{c}}$ :

$S_{\text {aper }}^{\text {tot }}=S_{\text {aper,u }}^{\text {source }}+S_{\text {aper,u }}^{\mathrm{bg}}=S_{\text {aper,c }}^{\text {source }}+S_{\text {aper,c }}^{\mathrm{bg}}$.

With Eq. (A.2) both the uncorrected and corrected background signal in the $120^{\prime \prime}$ aperture can be expressed as

$S_{120, \mathrm{u} / \mathrm{c}}^{\mathrm{bg}}=S_{180, \mathrm{u} / \mathrm{c}}^{\mathrm{bg}} \cdot \frac{A_{120}}{A_{180}} \cdot a_{120}^{\mathrm{corr}}$.

The corrected source signal in the $120^{\prime \prime}$ aperture is scaled with regard to the one in the $180^{\prime \prime}$ aperture via the model intensity ratio

$S_{120, c}^{\text {source }}=\frac{I_{120}^{\text {model }}}{I_{180}^{\text {model }}} \cdot S_{180, \mathrm{c}}^{\text {source }}$.

Using $A^{*}=\frac{A_{120}}{A_{180}} \cdot a_{120}^{\text {corr }}$, the total signal in the $120^{\prime \prime}$ aperture can then be expressed as

$S_{120}^{\text {tot }}=\frac{I_{120}^{\text {model }}}{I_{180}^{\text {model }}} \cdot S_{180, \mathrm{c}}^{\text {source }}+A^{*} \cdot S_{180, \mathrm{c}}^{\mathrm{bg}}$.

The total signal in the $180^{\prime \prime}$ aperture, $S_{180}^{\text {tot }}=S_{180, \mathrm{c}}^{\text {source }}+S_{180, \mathrm{c}}^{\mathrm{bg}}$ is scaled with $A^{*}$ yielding

$A^{*} \cdot S_{180}^{\text {tot }}=A^{*} \cdot S_{180, \mathrm{c}}^{\text {source }}+A^{*} \cdot S_{180, \mathrm{c}}^{\mathrm{bg}}$.
Subtracting Eq. (A.28) from Eq. (A.27) gives

$S_{120}^{\text {tot }}-A^{*} \cdot S_{180}^{\text {tot }}=\frac{I_{120}^{\text {model }}}{I_{180}^{\text {model }}} \cdot S_{180, \mathrm{c}}^{\text {source }}-A^{*} \cdot S_{180, \mathrm{c}}^{\text {source }}$.

Solving this equation for $S_{180, c}^{\text {source }}$ and replacing the total signals by the sums of the uncorrected signals of the source and the background yields

$S_{180, \mathrm{c}}^{\text {source }}=\frac{\left(S_{120, \mathrm{u}}^{\text {source }}+S_{120, \mathrm{u}}^{\mathrm{bg}}\right)-A^{*} \cdot\left(S_{180, \mathrm{u}}^{\text {source }}+S_{180, \mathrm{u}}^{\mathrm{bg}}\right)}{\frac{I_{120}^{\text {model }}}{I_{180}^{\text {model }}}-A^{*}}$.

The variation of the model intensity ratio $\Delta \frac{I_{120}^{\text {model }}}{I_{180}^{\text {model }}}$, within the error bars of the measured profile, is an additional uncertainty factor of the corrected flux density $F_{v, 180}^{\text {corr }}$. 
Table A.6. Observations in ISOPHOT multi-aperture mode PHT04 for which the source profile technique can be applied. This required measurements in the 120" and 180" aperture and at least 2 smaller apertures. The ISO TDT no. is a unique identifier of each observation. The different mission phases of ISO are indicated by $\mathrm{SC}=$ Satellite Commissioning Phase, $\mathrm{PV}=$ Performance Verification Phase and RT $=$ Routine Phase (execution of the science program); an additional $\mathrm{C}$ denotes calibration observations.

\begin{tabular}{|c|c|c|c|c|c|c|c|c|c|c|c|c|c|c|}
\hline \multirow[t]{2}{*}{ Target } & \multirow{2}{*}{$\begin{array}{l}\text { ISO TDT } \\
\text { No. }\end{array}$} & \multirow{2}{*}{$\begin{array}{c}\lambda_{\mathrm{c}} \\
(\mu \mathrm{m})\end{array}$} & \multirow[b]{2}{*}{$\begin{array}{l}5^{\prime \prime} \\
(s)\end{array}$} & \multirow[b]{2}{*}{$\begin{array}{l}7 " .6 \\
(\mathrm{~s})\end{array}$} & \multicolumn{7}{|c|}{ Exposure time per aperture / transient flag } & \multirow[b]{2}{*}{$\begin{array}{l}120^{\prime \prime} \\
(\mathrm{s})\end{array}$} & \multirow[b]{2}{*}{$\begin{array}{c}180^{\prime \prime} \\
(\mathrm{s})\end{array}$} & \multirow[t]{2}{*}{ Comment } \\
\hline & & & & & $\begin{array}{l}10^{\prime \prime} \\
(\mathrm{s})\end{array}$ & $\begin{array}{c}13^{\prime \prime} 8 \\
\text { (s) }\end{array}$ & $\begin{array}{l}18^{\prime \prime} \\
(\mathrm{s})\end{array}$ & $\begin{array}{c}23^{\prime \prime} \\
(\mathrm{s})\end{array}$ & $\begin{array}{l}52^{\prime \prime} \\
(\mathrm{s})\end{array}$ & $\begin{array}{l}79^{\prime \prime} \\
(\mathrm{s})\end{array}$ & $\begin{array}{l}99^{\prime \prime} \\
(\mathrm{s})\end{array}$ & & & \\
\hline NGC 6090 & 01301302 & 12 & - & 64 & 64 & 64 & 64 & 64 & 64 & 64 & 64 & 64 & $64^{t}$ & $\mathrm{SC}$ \\
\hline NGC 6090 & 01301402 & 12 & - & 64 & 64 & 64 & 64 & 64 & 64 & 64 & 64 & $64^{t}$ & 64 & $\mathrm{SC}$ \\
\hline NGC 6543 & 05702401 & 3.6 & $64^{t}$ & 64 & 64 & 64 & 64 & 64 & 64 & $64^{t}$ & 64 & 64 & 64 & $\mathrm{PV}$, commissioning \\
\hline NGC 6543 & 05702402 & 12 & - & - & 64 & 64 & 64 & 64 & 64 & $64^{t}$ & 64 & $64^{t}$ & $64^{t}$ & $\mathrm{PV}$, commissioning \\
\hline NGC 6543 & 05702403 & 60 & - & - & - & - & - & - & 64 & 64 & 64 & 64 & 64 & $\mathrm{PV}$, commissioning \\
\hline HR 6705 & 05702505 & 3.6 & 32 & 32 & 32 & 32 & 32 & 32 & 32 & 32 & 32 & 32 & 32 & PVC, PS standard \\
\hline HR 6705 & 05702506 & 12 & - & - & 32 & 32 & 32 & 32 & 32 & 32 & 32 & 32 & 32 & PVC, PS standard \\
\hline NGC 7008 & 05702610 & 25 & - & - & - & - & - & 64 & 64 & 64 & 64 & 64 & 64 & $\mathrm{PV}$, commissioning \\
\hline NGC 7008 & 05702611 & 60 & - & - & - & - & - & - & $64^{t}$ & 64 & 64 & 64 & 64 & $\mathrm{PV}$, commissioning \\
\hline HR 6705 & 06101501 & 25 & - & - & - & - & - & 32 & 32 & 32 & 32 & 32 & 32 & PVC, PS standard \\
\hline HD 97048 & 08400325 & 60 & - & - & - & - & - & - & 32 & 32 & 32 & 32 & 32 & RT \\
\hline RS Lib & 08402042 & 60 & - & - & - & - & - & - & $32^{t}$ & 32 & 32 & $32^{t}$ & 32 & RT \\
\hline $\mathrm{R} \mathrm{CrB}$ & 08500516 & 60 & - & - & - & - & - & - & 32 & $32^{t}$ & 32 & $32^{t}$ & 32 & $\mathrm{RT}$ \\
\hline WX Ser & 08500730 & 60 & - & - & - & - & - & - & 32 & 32 & 32 & 32 & 32 & $\mathrm{RT}$ \\
\hline $51 \mathrm{Oph}$ & 09100503 & 60 & - & - & - & - & - & - & $32^{t}$ & $32^{t}$ & $32^{t}$ & $32^{t}$ & 32 & RT \\
\hline HD 135344 & 10401743 & 15 & - & - & - & 32 & 32 & 32 & 32 & 32 & 32 & 32 & $32^{t}$ & $\mathrm{RT}$ \\
\hline HD 135344 & 10401744 & 60 & - & - & - & - & - & - & 32 & 32 & 32 & 32 & 32 & RT \\
\hline HD 139664 & 10402178 & 15 & - & - & - & 32 & 32 & 32 & 32 & 32 & 32 & 32 & 32 & $\mathrm{RT}$ \\
\hline HD 139664 & 10402179 & 60 & - & - & - & - & - & - & 32 & 32 & 32 & 32 & 32 & RT \\
\hline HD 139614 & 10402323 & 15 & - & - & - & 32 & 32 & 32 & 32 & $32^{t}$ & 32 & 32 & 32 & $\mathrm{RT}$ \\
\hline HD 139614 & 10402324 & 60 & - & - & - & - & - & - & 32 & 32 & 32 & 32 & 32 & RT \\
\hline HD 144432 & 10402663 & 15 & - & - & - & 32 & 32 & 32 & 32 & 32 & 32 & 32 & $32^{t}$ & $\mathrm{RT}$ \\
\hline HD 144432 & 10402664 & 60 & - & - & - & - & - & - & 32 & 32 & 32 & 32 & 32 & $\mathrm{RT}$ \\
\hline HD 142666 & 10402848 & 15 & - & - & - & 32 & 32 & 32 & 32 & 32 & 32 & 32 & $32^{t}$ & RT \\
\hline HD 142666 & 10402849 & 60 & - & - & - & - & - & - & 32 & 32 & 32 & 32 & 32 & RT \\
\hline HR 7001 & 10601103 & 25 & - & - & - & - & - & 32 & 32 & 32 & $32^{t}$ & 32 & $32^{t}$ & $\mathrm{RT}$ \\
\hline HR 7001 & 10601104 & 60 & - & - & - & - & - & - & 32 & 32 & 32 & 32 & $32^{t}$ & $\mathrm{RT}$ \\
\hline V Aql & 12100841 & 60 & - & - & - & - & - & - & 32 & 32 & 32 & 32 & 32 & RT \\
\hline $19521-5131$ & 12401516 & 25 & - & - & - & - & - & 32 & - & - & $32^{t}$ & $32^{t}$ & $32^{t}$ & RT \\
\hline $20174-7853$ & 12501222 & 25 & - & - & - & - & - & 32 & - & - & $32^{t}$ & $32^{t}$ & 32 & $\mathrm{RT}$ \\
\hline HD 188037 & 13101188 & 15 & - & - & - & 32 & 32 & 32 & 32 & 32 & $32^{t}$ & 32 & 32 & $\mathrm{RT}$ \\
\hline HD 188037 & 13101189 & 60 & - & - & - & - & - & - & 32 & 32 & 32 & 32 & 32 & $\mathrm{RT}$ \\
\hline $20270-2858$ & 13500328 & 25 & - & - & - & - & - & 32 & - & - & 32 & $32^{t}$ & 32 & $\mathrm{RT}$ \\
\hline HD 169142 & 13601438 & 15 & - & - & - & 32 & 32 & 32 & 32 & 32 & 32 & 32 & $32^{t}$ & RT \\
\hline HD 169142 & 13601439 & 60 & - & - & - & - & - & - & 32 & 32 & 32 & 32 & 32 & RT \\
\hline N 3998 & 14301342 & 20 & - & - & - & - & - & 128 & $128^{t}$ & 128 & 128 & 128 & 128 & RT \\
\hline $20240-2142$ & 14400425 & 25 & - & - & - & - & - & 32 & - & - & $32^{t}$ & 32 & 32 & RT \\
\hline HR 8728 & 16402913 & 25 & - & - & - & - & - & $32^{t}$ & 32 & 32 & 32 & 32 & 32 & $\mathrm{RT}$ \\
\hline HR 8728 & 16402914 & 60 & - & - & - & - & - & - & $32^{t}$ & 32 & $32^{t}$ & 32 & 32 & RT \\
\hline $09371+1212$ & 16900507 & 60 & - & - & - & - & - & - & 32 & 32 & 32 & 32 & 32 & $\mathrm{RT}$ \\
\hline N 5173 & 17201309 & 20 & - & - & - & - & - & 128 & 128 & $128^{t}$ & 128 & 128 & 128 & $\mathrm{RT}$ \\
\hline $22296+2004$ & 17301031 & 25 & - & - & - & - & - & 32 & - & - & 32 & 32 & 32 & RT \\
\hline HR 4534 & 18401559 & 60 & - & - & - & - & - & - & 32 & 32 & 32 & $32^{t}$ & 32 & RT \\
\hline HD 213985 & 18600322 & 60 & - & - & - & - & - & - & $32^{t}$ & 32 & 32 & 32 & $32^{t}$ & RT \\
\hline HR 4534 & 22700258 & 25 & - & - & - & - & - & 32 & 32 & 32 & 32 & 32 & 32 & $\mathrm{RT}$ \\
\hline HR 4534 & 22700259 & 60 & - & - & - & - & - & - & 32 & 32 & 32 & 32 & 32 & RT \\
\hline N 4261 & 23900143 & 20 & - & - & - & - & - & 128 & 128 & 128 & 128 & 128 & 128 & RT \\
\hline N 4486 & 23901503 & 20 & - & - & - & - & - & 128 & 128 & 128 & 128 & 128 & 128 & $\mathrm{RT}$ \\
\hline $\mathrm{N} 4552$ & 23902004 & 20 & - & - & - & - & - & $128^{t}$ & 128 & 128 & $128^{t}$ & 128 & 128 & RT \\
\hline N 4374 & 23902245 & 20 & - & - & - & - & - & 128 & 128 & 128 & $128^{t}$ & 128 & 128 & $\mathrm{RT}$ \\
\hline HD 98800 & 24001018 & 15 & - & - & - & 32 & 32 & 32 & 32 & 32 & 32 & 32 & $32^{t}$ & RT \\
\hline HD 98800 & 24001019 & 60 & - & - & - & - & - & - & $32^{t}$ & $32^{t}$ & $32^{t}$ & 32 & 32 & $\mathrm{RT}$ \\
\hline
\end{tabular}


Table A.6. continued.

\begin{tabular}{|c|c|c|c|c|c|c|c|c|c|c|c|c|c|c|}
\hline \multirow[t]{2}{*}{ Target } & \multirow{2}{*}{$\begin{array}{l}\text { ISO TDT } \\
\text { No. }\end{array}$} & \multirow{2}{*}{$\begin{array}{c}\lambda_{\mathrm{c}} \\
(\mu \mathrm{m})\end{array}$} & \multicolumn{11}{|c|}{ Exposure time per aperture / transient flag } & \multirow[t]{2}{*}{ Comment } \\
\hline & & & $\begin{array}{l}5^{\prime \prime} \\
(\mathrm{s})\end{array}$ & $\begin{array}{l}7{ }^{\prime \prime} 6 \\
\text { (s) }\end{array}$ & $\begin{array}{c}10^{\prime \prime} \\
(\mathrm{s})\end{array}$ & $\begin{array}{c}133^{\prime \prime} 8 \\
\text { (s) }\end{array}$ & $\begin{array}{c}18^{\prime \prime} \\
(\mathrm{s})\end{array}$ & $\begin{array}{c}23^{\prime \prime} \\
(\mathrm{s})\end{array}$ & $\begin{array}{c}52^{\prime \prime} \\
(\mathrm{s})\end{array}$ & $\begin{array}{c}79^{\prime \prime} \\
(\mathrm{s})\end{array}$ & $\begin{array}{c}99^{\prime \prime} \\
(\mathrm{s})\end{array}$ & $\begin{array}{c}120^{\prime \prime} \\
(\mathrm{s})\end{array}$ & $\begin{array}{c}180^{\prime \prime} \\
(\mathrm{s})\end{array}$ & \\
\hline R Cas & 26300812 & 60 & - & - & - & - & - & - & 32 & 32 & 32 & 32 & 32 & RT \\
\hline HR 21 & 28200973 & 25 & - & - & - & - & - & 32 & 32 & 32 & $32^{t}$ & 32 & $32^{t}$ & RT \\
\hline HR 21 & 28200974 & 60 & - & - & - & - & - & - & 32 & 32 & 32 & 32 & $32^{t}$ & RT \\
\hline N 1052 & 28302633 & 20 & - & - & - & - & - & 128 & 128 & 128 & $128^{t}$ & 128 & 128 & RT \\
\hline V854 Cen & 29701055 & 60 & - & - & - & - & - & - & 32 & 32 & 32 & 32 & 32 & RT \\
\hline T Dra & 30200832 & 60 & - & - & - & - & - & - & 32 & 32 & 32 & $32^{t}$ & 32 & $\mathrm{RT}$ \\
\hline HR 8728 & 35300713 & 25 & - & - & - & - & - & 32 & $32^{t}$ & 32 & 32 & 32 & 32 & RT \\
\hline HR 8728 & 35300714 & 60 & - & - & - & - & - & - & $32^{t}$ & 32 & 32 & 32 & 32 & RT \\
\hline YCVn & 35700412 & 60 & - & - & - & - & - & - & 32 & 32 & 32 & 32 & 32 & RT \\
\hline$\chi$ Cyg & 35700824 & 60 & - & - & - & - & - & - & 32 & 32 & 32 & 32 & 32 & $\mathrm{RT}$ \\
\hline HD 179218 & 36400888 & 60 & - & - & 32 & 32 & 32 & 32 & 32 & 32 & 32 & 32 & 32 & RT \\
\hline OH104.9+2.4 & 36802046 & 60 & - & - & - & - & - & - & 32 & 32 & 32 & 32 & 32 & RT \\
\hline HR 451 & 39300928 & 25 & - & - & - & - & - & 32 & 32 & $32^{t}$ & $32^{t}$ & 32 & 32 & RT \\
\hline $\mathrm{HR} 451$ & 39300929 & 60 & - & - & - & - & - & - & 32 & 32 & 32 & 32 & $32^{t}$ & RT \\
\hline HR 509 & 39301183 & 25 & - & - & - & - & - & 32 & 32 & 32 & 32 & 32 & 32 & RT \\
\hline HR 509 & 39301184 & 60 & - & - & - & - & - & - & 32 & 32 & 32 & 32 & 32 & RT \\
\hline CRL 3068 & 39500607 & 15 & - & - & - & 32 & 32 & 32 & 32 & 32 & 32 & 32 & 32 & $\mathrm{RT}$ \\
\hline N 5363 & 39600411 & 20 & - & - & - & - & - & 128 & 128 & $128^{t}$ & 128 & 128 & 128 & RT \\
\hline HR 818 & 40101833 & 25 & - & - & - & - & - & 32 & 32 & $32^{t}$ & 32 & $32^{t}$ & 32 & RT \\
\hline HR 818 & 40101834 & 60 & - & - & - & - & - & - & $32^{t}$ & 32 & 32 & 32 & 32 & RT \\
\hline $\mathrm{R} \mathrm{CrB}$ & 41700944 & 60 & - & - & - & - & - & - & 64 & 64 & $64^{t}$ & 64 & 64 & RT \\
\hline HD 161796 & 41905010 & 60 & - & - & - & - & - & - & 64 & 64 & 64 & 64 & 64 & $\mathrm{RT}$ \\
\hline IRC+100011 & 42201916 & 15 & - & - & - & 32 & 32 & 32 & 32 & 32 & 32 & 32 & 32 & RT \\
\hline$\mu \mathrm{CEP}$ & 42604804 & 60 & - & - & - & - & - & - & 64 & 64 & 64 & 64 & 64 & RT \\
\hline $\mathrm{OH} 127.8+0.0$ & 43306020 & 60 & - & - & - & - & - & - & 64 & 64 & 64 & 64 & 64 & RT \\
\hline N 0855 & 44004832 & 20 & - & - & - & - & - & $128^{t}$ & 128 & 128 & 128 & 128 & 128 & $\mathrm{RT}$ \\
\hline N 0807 & 44004931 & 20 & - & - & - & - & - & 128 & 128 & 128 & 128 & 128 & 128 & RT \\
\hline N 5666 & 44400712 & 20 & - & - & - & - & - & 128 & $128^{t}$ & $128^{t}$ & 128 & 128 & 128 & $\mathrm{RT}$ \\
\hline Mira & 44602008 & 15 & - & - & - & 32 & 32 & 32 & 32 & 32 & 32 & 32 & 32 & RT \\
\hline $51 \mathrm{Oph}$ & 45602027 & 60 & - & - & - & - & - & - & 64 & 64 & 64 & 64 & 64 & $\mathrm{RT}$ \\
\hline HR 5999 & 45801043 & 7.3 & 32 & 32 & 32 & 32 & 32 & 32 & 32 & 32 & 32 & 32 & 32 & $\mathrm{RT}$ \\
\hline HR 5999 & 45801044 & 10 & 32 & 32 & 32 & 32 & 32 & 32 & 32 & 32 & 32 & 32 & $32^{t}$ & RT \\
\hline HR 5999 & 45801047 & 20 & - & - & 32 & 32 & 32 & 32 & 32 & 32 & 32 & 32 & 32 & $\mathrm{RT}$ \\
\hline HR 5999 & 45801048 & 60 & - & - & 32 & 32 & 32 & $32^{t}$ & 32 & 32 & 32 & 32 & $32^{t}$ & RT \\
\hline HR 6000 & 45801253 & 7.3 & 32 & 32 & 32 & 32 & 32 & $32^{t}$ & 32 & 32 & $32^{t}$ & $32^{t}$ & $32^{t}$ & $\mathrm{RT}$ \\
\hline HR 6000 & 45801254 & 10 & 32 & 32 & 32 & 32 & 32 & 32 & 32 & 32 & $32^{t}$ & 32 & $32^{t}$ & RT \\
\hline HR 6000 & 45801257 & 20 & - & - & 32 & 32 & 32 & 32 & $32^{t}$ & $32^{t}$ & 32 & 32 & 32 & RT \\
\hline HR 6000 & 45801258 & 60 & - & - & 32 & 32 & 32 & 32 & 32 & $32^{t}$ & 32 & 32 & $32^{t}$ & $\mathrm{RT}$ \\
\hline $16342-3814$ & 45801419 & 15 & - & - & - & 32 & 32 & 32 & 32 & 32 & 32 & 32 & $32^{t}$ & RT \\
\hline VX Sgr & 47000926 & 15 & - & - & - & 32 & 32 & 32 & 32 & 32 & 32 & 32 & $32^{t}$ & $\mathrm{RT}$ \\
\hline RY Sgr & 49302620 & 60 & - & - & - & - & - & - & 64 & 64 & 64 & 64 & 64 & RT \\
\hline HD 176386 & 52301433 & 7.3 & 32 & 32 & $32^{t}$ & $32^{t}$ & 32 & 32 & 32 & $32^{t}$ & $32^{t}$ & $32^{t}$ & $32^{t}$ & RT \\
\hline HD 176386 & 52301434 & 10 & 32 & 32 & 32 & 32 & 32 & 32 & 32 & 32 & 32 & $32^{t}$ & $32^{t}$ & RT \\
\hline HD 176386 & 52301437 & 20 & - & - & $32^{t}$ & $32^{t}$ & $32^{t}$ & $32^{t}$ & 32 & 32 & 32 & 32 & 32 & $\mathrm{RT}$ \\
\hline HD 176386 & 52301438 & 60 & - & - & 32 & 32 & 32 & 32 & $32^{t}$ & 32 & 32 & 32 & 32 & RT \\
\hline TY CrA & 52301523 & 7.3 & 32 & 32 & 32 & 32 & 32 & 32 & 32 & 32 & 32 & 32 & $32^{t}$ & $\mathrm{RT}$ \\
\hline TY CrA & 52301524 & 10 & 32 & 32 & 32 & 32 & 32 & 32 & 32 & 32 & 32 & 32 & $32^{t}$ & RT \\
\hline TY CrA & 52301527 & 20 & - & - & 32 & 32 & 32 & 32 & 32 & 32 & 32 & 32 & 32 & RT \\
\hline TY CrA & 52301528 & 60 & - & - & 32 & 32 & 32 & 32 & 32 & 32 & 32 & 32 & 32 & $\mathrm{RT}$ \\
\hline HD 179218 & 52502083 & 7.3 & 32 & 32 & 32 & 32 & 32 & 32 & 32 & 32 & $32^{t}$ & $32^{t}$ & $32^{t}$ & RT \\
\hline HD 179218 & 52502084 & 10 & 32 & 32 & 32 & 32 & 32 & 32 & 32 & 32 & 32 & 32 & $32^{t}$ & RT \\
\hline HD 179218 & 52502087 & 20 & - & - & 32 & 32 & 32 & 32 & 32 & 32 & 32 & 32 & 32 & RT \\
\hline HD 179218 & 52502088 & 60 & - & - & 32 & 32 & 32 & 32 & 32 & 32 & 32 & 32 & 32 & $\mathrm{RT}$ \\
\hline $19575-4317$ & 53401319 & 25 & - & - & - & - & - & 32 & - & - & $32^{t}$ & 32 & 32 & RT \\
\hline I 1459 & 54701313 & 20 & - & - & - & - & - & 128 & $128^{t}$ & $128^{t}$ & 128 & 128 & 128 & RT \\
\hline N 7768 & 56500520 & 20 & - & - & - & - & - & 128 & 128 & 128 & 128 & $128^{t}$ & 128 & $\mathrm{RT}$ \\
\hline
\end{tabular}


Table A.6. continued.

\begin{tabular}{|c|c|c|c|c|c|c|c|c|c|c|c|c|c|c|}
\hline \multirow[t]{2}{*}{ Target } & \multirow{2}{*}{$\begin{array}{l}\text { ISO TDT } \\
\text { No. }\end{array}$} & \multirow{2}{*}{$\begin{array}{c}\lambda_{\mathrm{c}} \\
(\mu \mathrm{m})\end{array}$} & \multirow[b]{2}{*}{$\begin{array}{l}5^{\prime \prime} \\
(\mathrm{s})\end{array}$} & \multicolumn{10}{|c|}{ Exposure time per aperture / transient flag } & \multirow[t]{2}{*}{ Comment } \\
\hline & & & & $\begin{array}{l}7 . \prime 6 \\
\text { (s) }\end{array}$ & $\begin{array}{l}10^{\prime \prime} \\
(\mathrm{s})\end{array}$ & $\begin{array}{c}13^{\prime \prime} 8 \\
\text { (s) }\end{array}$ & $\begin{array}{c}18^{\prime \prime} \\
(\mathrm{s})\end{array}$ & $\begin{array}{l}23^{\prime \prime} \\
(\mathrm{s})\end{array}$ & $\begin{array}{c}52^{\prime \prime} \\
(\mathrm{s})\end{array}$ & $\begin{array}{c}79^{\prime \prime} \\
(\mathrm{s})\end{array}$ & $\begin{array}{l}99^{\prime \prime} \\
(\mathrm{s})\end{array}$ & $\begin{array}{c}120^{\prime \prime} \\
(\mathrm{s})\end{array}$ & $\begin{array}{c}180^{\prime \prime} \\
(\mathrm{s})\end{array}$ & \\
\hline Bright Region 5 & 56600502 & 15 & - & - & - & - & - & - & 128 & 128 & 128 & $128^{t}$ & $128^{t}$ & RTC, flat sky \\
\hline I 2006 & 58500535 & 20 & - & - & - & - & - & 64 & 64 & 64 & 64 & $64^{t}$ & 64 & RT \\
\hline UW Cen & 60700417 & 60 & - & - & - & - & - & - & 32 & 32 & 32 & 32 & 32 & $\mathrm{RT}$ \\
\hline Bright Region 5 & 60802402 & 15 & - & - & - & - & - & - & 512 & 512 & 512 & $512^{t}$ & 512 & RTC, flat sky \\
\hline HR 2020 & 62402208 & 15 & - & - & - & 64 & 64 & 64 & 64 & 64 & 64 & 64 & $64^{t}$ & $\mathrm{RT}$ \\
\hline HR 2020 & 62402209 & 60 & - & - & - & - & - & - & 64 & 64 & 64 & 64 & 64 & RT \\
\hline HD 34700 & 63602295 & 15 & - & - & - & 64 & 64 & 64 & 64 & 64 & 64 & 64 & $64^{t}$ & $\mathrm{RT}$ \\
\hline HD 34700 & 63602296 & 60 & - & - & - & - & - & - & 64 & 64 & 64 & 64 & 64 & RT \\
\hline HD 97300 & 67901411 & 3.3 & 64 & 64 & 64 & 64 & 64 & 64 & 64 & 64 & 64 & 64 & 64 & RT \\
\hline HD 97300 & 67901412 & 4.8 & 32 & 32 & 32 & 32 & 32 & 32 & 32 & 32 & 32 & 32 & 32 & RT \\
\hline HD 97300 & 67901413 & 7.3 & 32 & 32 & 32 & 32 & $32^{t}$ & 32 & 32 & $32^{t}$ & $32^{t}$ & $32^{t}$ & $32^{t}$ & $\mathrm{RT}$ \\
\hline HD 97300 & 67901414 & 10 & $32^{t}$ & 32 & 32 & 32 & 32 & 32 & 32 & 32 & 32 & $32^{t}$ & $32^{t}$ & $\mathrm{RT}$ \\
\hline HD 97300 & 67901415 & 11.3 & 32 & 32 & 32 & 32 & 32 & 32 & 32 & 32 & 32 & 32 & $32^{t}$ & RT \\
\hline HD 97300 & 67901416 & 12.8 & 32 & 32 & 32 & 32 & 32 & 32 & 32 & 32 & 32 & $32^{t}$ & $32^{t}$ & $\mathrm{RT}$ \\
\hline HD 97300 & 67901417 & 15 & - & - & 32 & 32 & 32 & 32 & 32 & 32 & 32 & 32 & $32^{t}$ & RT \\
\hline HD 97300 & 67901418 & 60 & - & - & 32 & 32 & 32 & 32 & 32 & 32 & 32 & 32 & 32 & $\mathrm{RT}$ \\
\hline HD 97300 & 67901419 & 100 & - & - & 32 & 32 & 32 & 32 & 32 & 32 & 32 & 32 & 32 & RT \\
\hline HD 97300 & 70401010 & 20 & - & - & 32 & 32 & 32 & $32^{t}$ & 32 & 32 & 32 & 32 & 32 & $\mathrm{RT}$ \\
\hline HD 97300 & 70401020 & 25 & - & - & 32 & 32 & $32^{t}$ & 32 & 32 & 32 & 32 & 32 & 32 & $\mathrm{RT}$ \\
\hline HD 97048 & 70401603 & 7.3 & 32 & 32 & 32 & 32 & 32 & 32 & 32 & $32^{t}$ & $32^{t}$ & $32^{t}$ & $32^{t}$ & $\mathrm{RT}$ \\
\hline HD 97048 & 70401604 & 10 & 32 & 32 & 32 & 32 & 32 & 32 & $32^{t}$ & 32 & 32 & 32 & $32^{t}$ & $\mathrm{RT}$ \\
\hline HD 97048 & 70401607 & 20 & - & - & 32 & 32 & 32 & 32 & 32 & 32 & 32 & 32 & 32 & RT \\
\hline HD 97048 & 70401608 & 60 & - & - & 32 & 32 & 32 & 32 & 32 & 32 & 32 & 32 & 32 & $\mathrm{RT}$ \\
\hline W Aql & 71101028 & 60 & - & - & - & - & - & - & 64 & 64 & 64 & 64 & 64 & RT \\
\hline Bright Region 6 & 71301001 & 7.3 & - & - & - & - & - & 512 & 512 & 512 & 512 & 512 & 512 & RTC, flat sky \\
\hline Bright Region 6 & 71301103 & 12 & - & - & - & - & - & - & 256 & 256 & 256 & 256 & 256 & RTC, flat sky \\
\hline Bright Region 6 & 71301204 & 25 & - & - & - & - & - & - & - & 256 & 256 & 256 & 256 & RTC, flat sky \\
\hline Bright Region 6 & 71302105 & 60 & - & - & - & - & - & - & - & 256 & 256 & 256 & 256 & RTC, flat sky \\
\hline$\iota \mathrm{Gru}$ & 71800733 & 20 & - & - & - & - & - & $64^{t}$ & 64 & 64 & 64 & 64 & 64 & RT \\
\hline$\gamma$ Tuc & 71801434 & 20 & - & - & - & - & - & 64 & $64^{t}$ & 64 & $64^{t}$ & $64^{t}$ & 64 & $\mathrm{RT}$ \\
\hline T Cha & 71901773 & 7.3 & 32 & 32 & 32 & 32 & 32 & 32 & 32 & 32 & 32 & 32 & 32 & RT \\
\hline TCha & 71901774 & 10 & 32 & 32 & 32 & 32 & 32 & 32 & 32 & 32 & 32 & 32 & $32^{t}$ & $\mathrm{RT}$ \\
\hline TCha & 71901777 & 20 & - & - & 32 & 32 & 32 & 32 & $32^{t}$ & 32 & $32^{t}$ & $32^{t}$ & 32 & RT \\
\hline T Cha & 71901778 & 60 & - & - & 32 & 32 & 32 & 32 & 32 & 32 & 32 & 32 & 32 & $\mathrm{RT}$ \\
\hline HR 7980 off & 72002401 & 25 & - & - & - & - & - & $128^{t}$ & 128 & 128 & 128 & 128 & 128 & RTC, flat sky \\
\hline HR 7980 & 72002402 & 25 & - & - & - & - & - & 128 & 128 & 128 & 128 & 128 & 128 & RTC, PS standard \\
\hline HR 13 & 73500731 & 20 & - & - & - & - & - & 64 & $64^{t}$ & 64 & $64^{t}$ & $64^{t}$ & 64 & RT \\
\hline $03287-1535$ & 78000601 & 12 & - & - & 32 & - & 32 & - & $32^{t}$ & - & - & 32 & 32 & $\mathrm{RT}$ \\
\hline 03287-1535 & 78000611 & 25 & - & - & - & - & - & 32 & 32 & - & - & 32 & 32 & $\mathrm{RT}$ \\
\hline HR 6705 off & 79600205 & 60 & - & - & - & - & - & $128^{t}$ & 128 & 128 & 128 & 128 & 128 & RTC, flat sky \\
\hline HR 6705 & 79600206 & 60 & - & - & 128 & 128 & $128^{t}$ & 128 & 128 & 128 & 128 & 128 & 128 & RTC, PS standard \\
\hline HR 1084 & 79601207 & 60 & - & - & - & - & - & - & 64 & 64 & 64 & 64 & 64 & RT \\
\hline N 1172 & 79601517 & 20 & - & - & - & - & - & 128 & 128 & $128^{t}$ & $128^{t}$ & 128 & 128 & RT \\
\hline HR 617 off & 80500701 & 25 & - & - & - & - & - & 128 & 128 & 128 & 128 & 128 & 128 & RTC, flat sky \\
\hline HR 617 & 80500702 & 25 & - & - & - & - & - & 128 & 128 & 128 & 128 & 128 & 128 & RTC, PS standard \\
\hline HR 617 off & 80500803 & 20 & - & - & 128 & 128 & 128 & 128 & 128 & 128 & 128 & 128 & 128 & RTC, flat sky \\
\hline HR 617 & 80500804 & 20 & - & - & 128 & 128 & 128 & $128^{t}$ & 128 & 128 & 128 & 128 & 128 & RTC, PS standard \\
\hline HR 337 off & 80501507 & 60 & - & - & - & - & $128^{t}$ & 128 & 128 & 128 & 128 & 128 & 128 & RTC, flat sky \\
\hline HR 337 & 80501508 & 60 & - & - & 128 & 128 & 128 & 128 & 128 & 128 & 128 & 128 & 128 & RTC, PS standard \\
\hline HR 1457 off & 81500709 & 60 & - & - & 128 & 128 & 128 & 128 & 128 & 128 & 128 & 128 & 128 & RTC, flat sky \\
\hline HR 1457 & 81500710 & 60 & - & - & 128 & 128 & 128 & 128 & 128 & 128 & 128 & 128 & 128 & RTC, PS standard \\
\hline HR 7127 off & 87500103 & 7.3 & - & - & - & 128 & 128 & 128 & 128 & 128 & 128 & 128 & $128^{t}$ & RTC, flat sky \\
\hline HR 7127 & 87500104 & 7.3 & 128 & 128 & 128 & 128 & 128 & 128 & 128 & 128 & 128 & 128 & 128 & RTC, PS standard \\
\hline
\end{tabular}

${ }^{t}$ Data quality report of this TDT flags non-stable signals due to transients for this aperture. 
U. Klaas et al.: ISOPHOT multi-aperture photometry. I., Online Material p 18
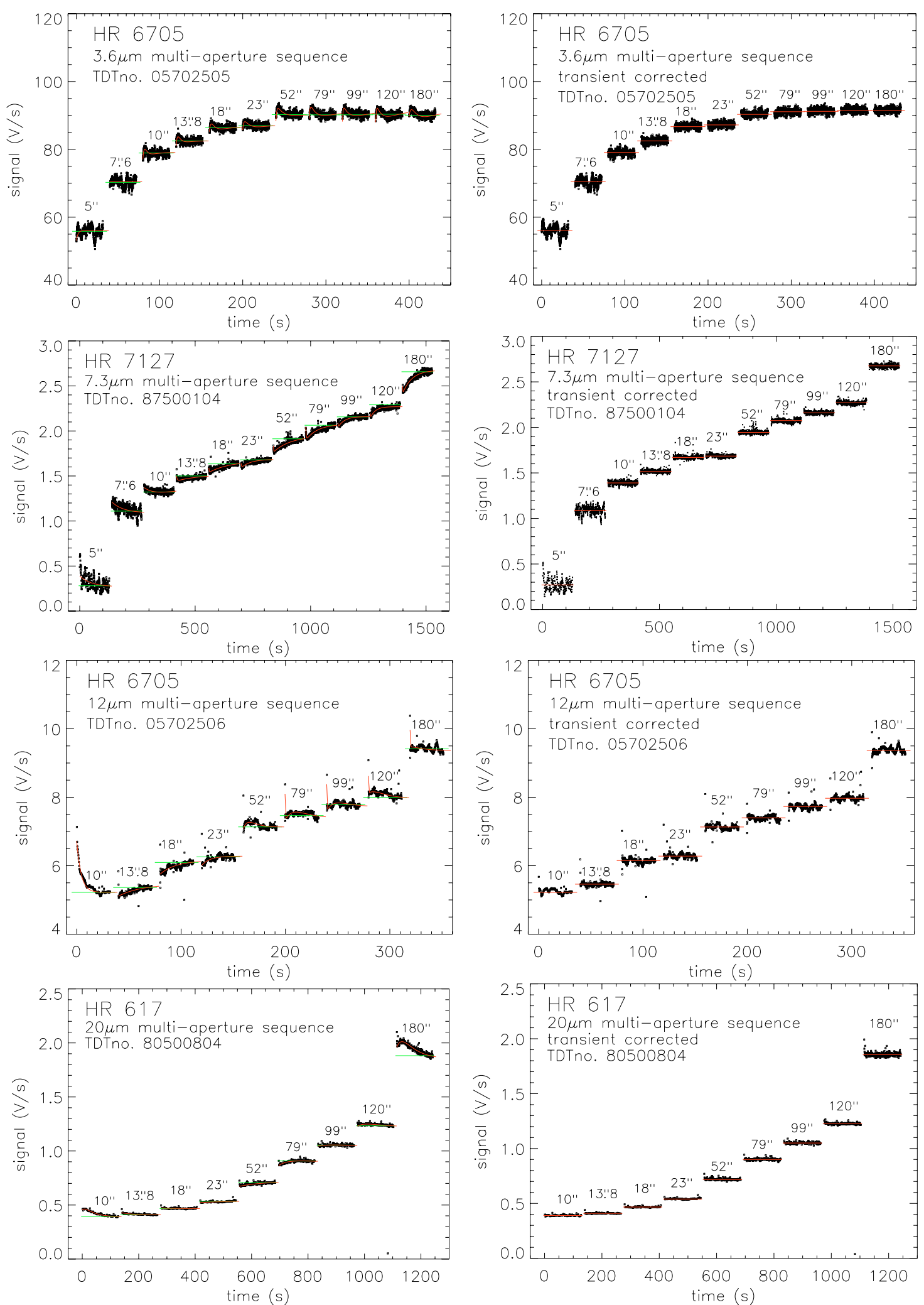

Fig. A.12. Signal time series of multi-aperture sequences of the point source standards HR 6705 at $3.6 \mu \mathrm{m}$ and $12 \mu \mathrm{m}, \mathrm{HR} 7127$ at $7.3 \mu \mathrm{m}$ and HR 617 at $20 \mu \mathrm{m}$. The lefthand panels show uncorrected signals with the green lines indicating the final signal levels predicted by the standard drift recognition and the red lines indicating the transient fits. The righthand panels show transient corrected signals with the final signal level at infinity indicated by the red line. 
U. Klaas et al.: ISOPHOT multi-aperture photometry. I., Online Material $p 19$
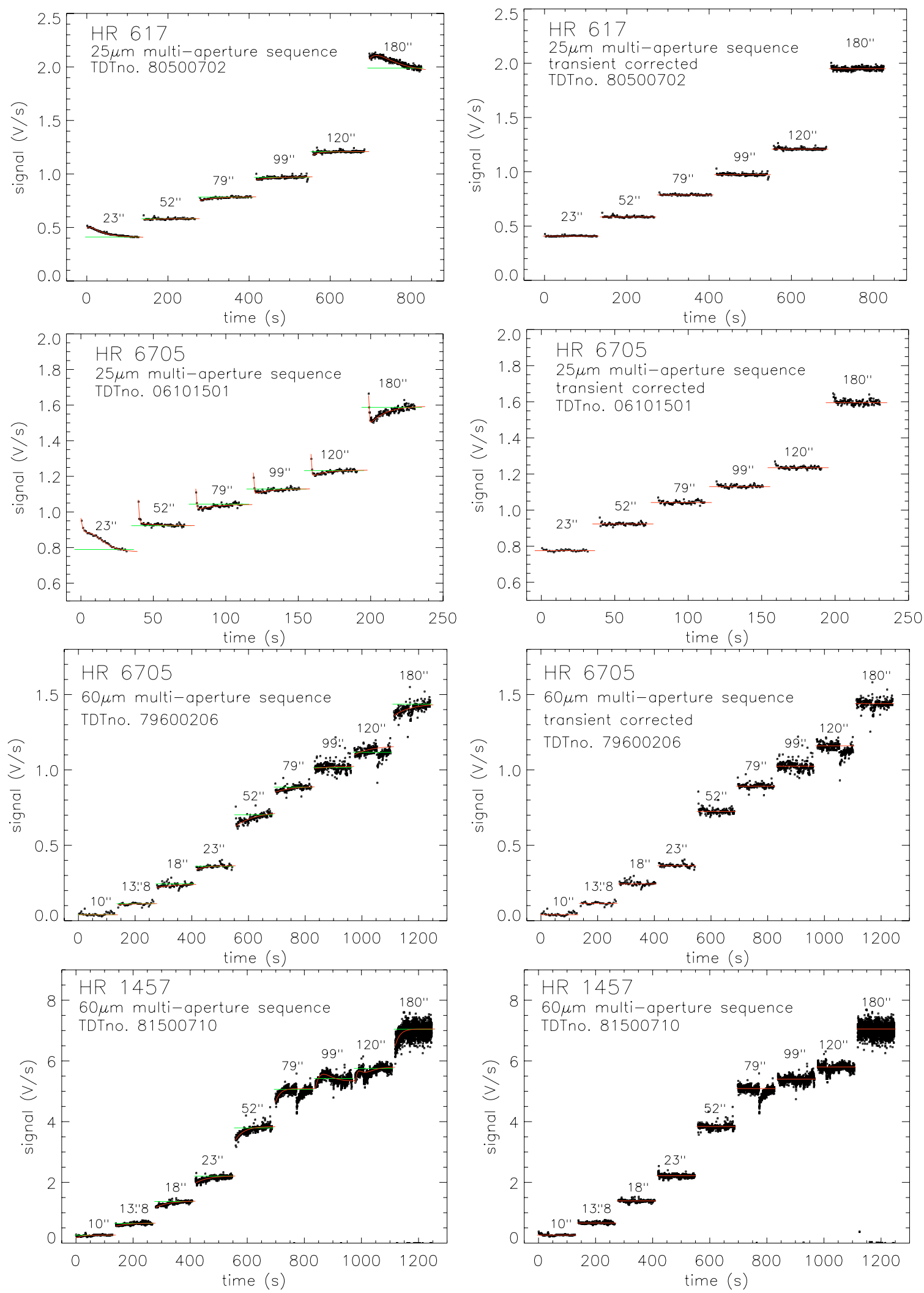

Fig. A.12. continued. Signal time series of multi-aperture sequences of the point source standards HR 617 at $25 \mu \mathrm{m}$, HR 6705 at $25 \mu \mathrm{m}$ and $60 \mu \mathrm{m}$, HR 1457 at $60 \mu \mathrm{m}$. 